\title{
ADHERENS JUNCTION ENGAGEMENT REGULATES FUNCTIONAL PATTERNING OF THE CARDIAC PACEMAKER CELL LINEAGE
}

\author{
Kandace Thomas
}

A dissertation submitted to the faculty at the University of North Carolina at Chapel Hill in partial fulfillment of the requirements for the degree of Doctorate of Philosophy in the Genetics and Molecular Biology in the School of Medicine.

Chapel Hill

2021

Approved by:

Michael Bressan

Frank Conlon

Victoria Bautch

Li Qian

Mauro Calabrese 
(C) 2021

Kandace Thomas

ALL RIGHTS RESERVED 


\begin{abstract}
Kandace Thomas: Adherens Junction Engagement Regulates Functional Patterning of the Cardiac Pacemaker Cell Lineage (Under the direction of Michael Bressan)
\end{abstract}

The heart is the first organ system to form in the embryo. Over the course of development, cardiomyocytes with differing morphogenetic, molecular, and physiological characteristics are specified and differentiate and integrate with one another to assemble a coordinated electromechanical pumping system that can function independently of any external stimulus. As congenital malformation of the heart presents the leading class of birth defects seen in humans, the molecular genetics of heart development have garnered much attention over the last half century. However, understanding how genetic perturbations manifest at the level of the individual cell function remains challenging to investigate. In this dissertation, we briefly introduce the history of imaging approaches for assessing cardiac development, describe some of the reagents and tools required to perform live imaging in the developing heart, and discuss how the combination of modern imaging modalities and physiological probes can be used to scale from subcellular to whole-organ analysis. Through these types of imaging approaches, critical insights into the processes of cardiac physiological development can be directly examined in realtime. Moving forward, the synthesis of modern molecular biology and imaging approaches will open novel avenues to investigate the mechanisms of cardiomyocyte maturation.

Specifically, cardiac pacemaker cells (CPCs) will be discussed in detail. CPCs rhythmically initiate the electrical impulses that drive heart contraction. CPCs display the highest rate of spontaneous depolarization in the heart despite being subjected to inhibitory electrochemical conditions that should theoretically suppress their activity. While several models have been proposed to explain this apparent paradox, the actual molecular mechanisms that allow CPCs to overcome electrogenic barriers to their 
function remain poorly understood. Here, we have traced CPC development at single-cell resolution and uncovered a series of cytoarchitectural patterning events that are critical for proper pacemaking.

Specifically, our data reveal that CPCs dynamically modulate adherens junction (AJ) engagement to control characteristics including surface area, volume, and gap junctional coupling. This allows CPCs to adopt a structural configuration that supports their overall excitability. Thus, our data have identified a direct role for local cellular mechanics in patterning critical morphological features that are necessary for CPC electrical activity. 
To my mentor and lab mates, I couldn't have done this without you. Thank you for all of your support along the way. 


\section{ACKNOWLEDGEMENTS}

Support for this work was provided by grants R00HL122360 to M.B. from the National Institutes of Health. As well as grants T32HL06976 (NHLBI) to K.T., UL1TR002489 (NCATS) to W.P., R01HL146626 (NHLBI) to M.B., and American Heart Association grants 19POST34380213 to R.S. and 19CDA34760248 to M.B. Imaging was performed in the Hooker Imaging Core Facility and Neuroscience Microscopy Core at the University of North Carolina (supported NIHNINDS P30 NS045892 and NIHNICHD U54 HD079124). 


\section{TABLE OF CONTENTS}

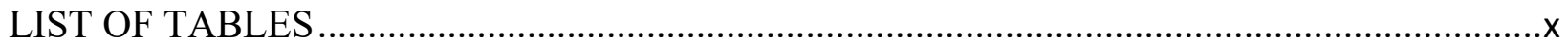

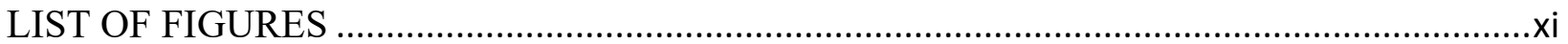

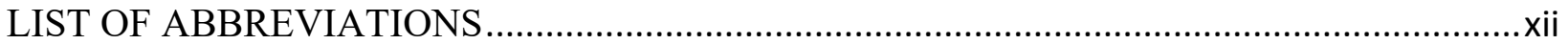

CHAPTER 1: THE HISTORY OF CARDIAC PACEMAKER CELLS ..................................... 1

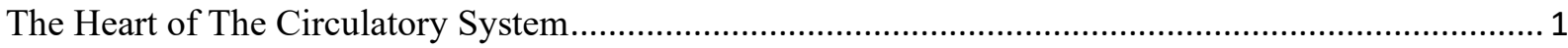

The Anatomy and Function of the Cardiac Conduction System ........................................................ 4

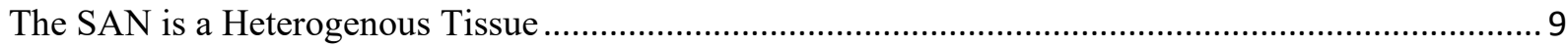

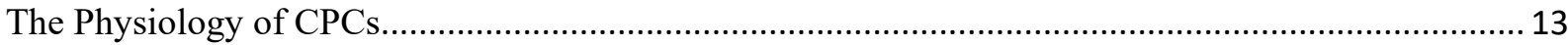

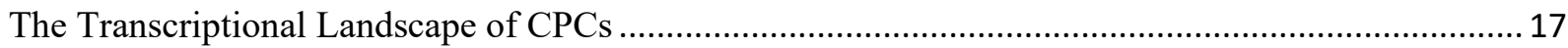

The Interplay Between CPC Cell Biology, Genetics, and Physiology .............................................. 19

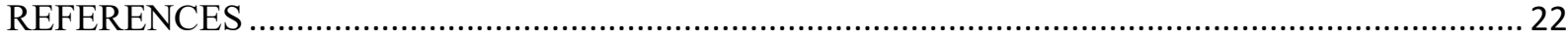

CHAPTER 2: OPTICAL ELECTROPHYSIOLOGY IN THE

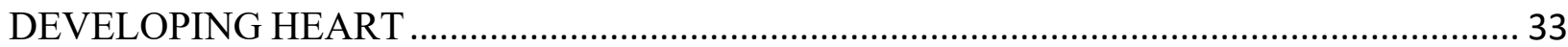

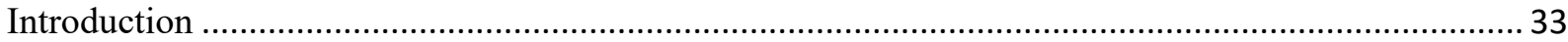

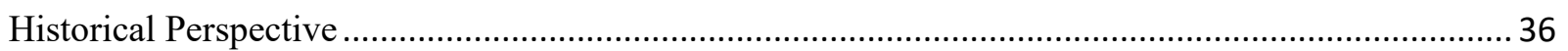

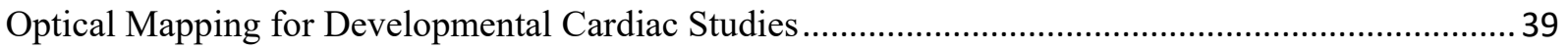

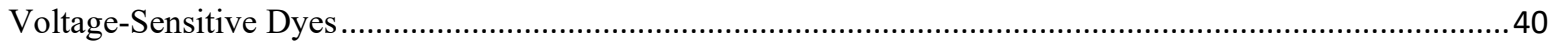

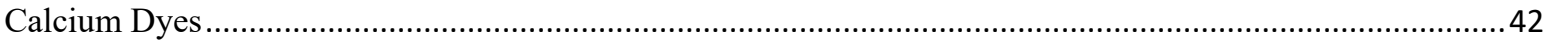

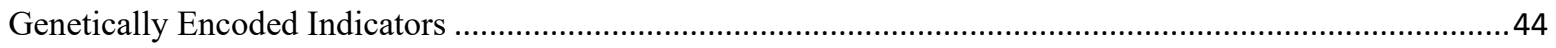

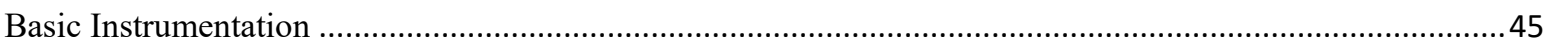

Application of Physiological Imaging to Understand Cardiac 


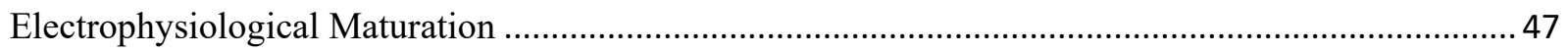

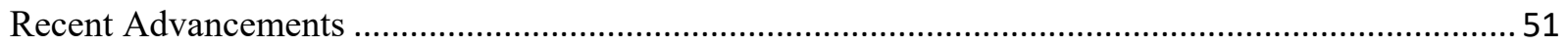

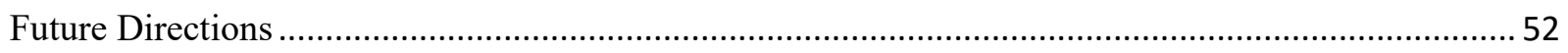

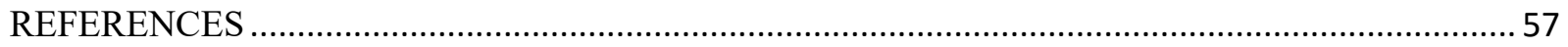

\section{CHAPTER 3: ADHERENS JUNCTION ENGAGEMENT}

REGULATES FUNCTIONAL PATTERNING OF THE CARDIAC

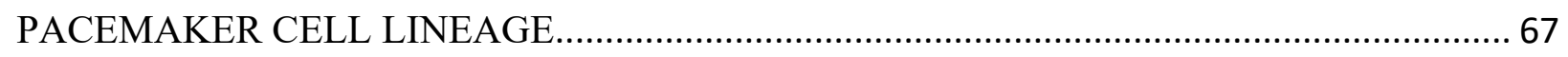

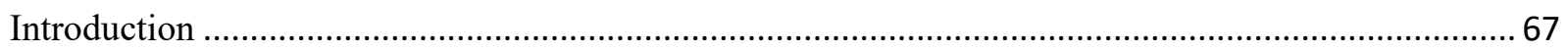

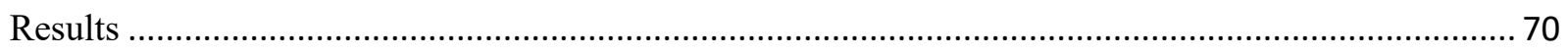

CPCs structurally diverge from the WM after becoming electrically active..................................................74

CPCs lose the ability to form AJ as they diverge from the WM ................................................................75

Inhibition of the cardiac AJ converts atrial WM to a CPC-like morphology ..................................................78

Force-mediated AJ engagement induces a WM-like phenotype in CPCs ....................................................78

AJ formation regulates CPC gap junction formation .................................................................................. 81

$\mathrm{CPC} \mathrm{AJ}$ engagement is regulated by regional enrichment of the

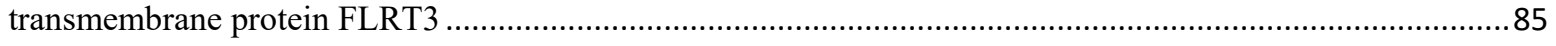

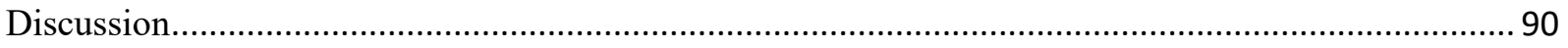

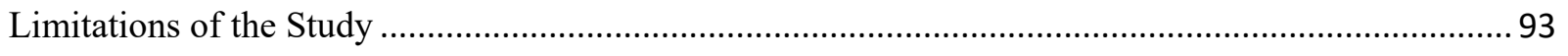

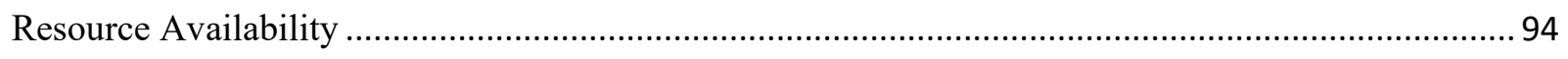

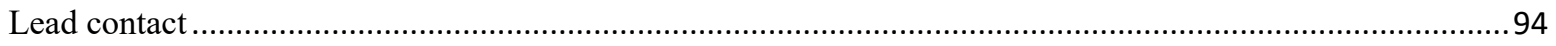

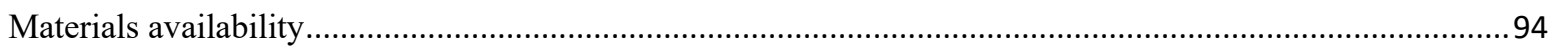

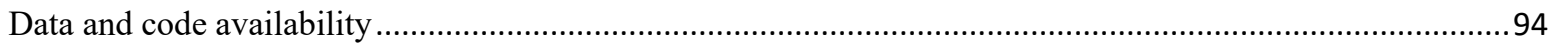

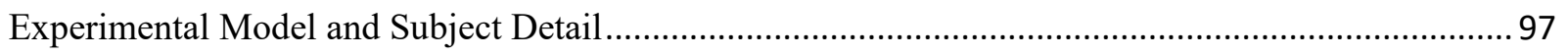

Animals …

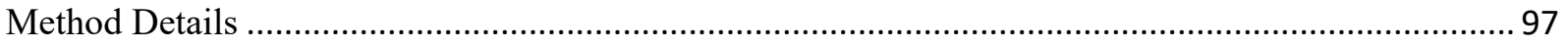

Transfection ……

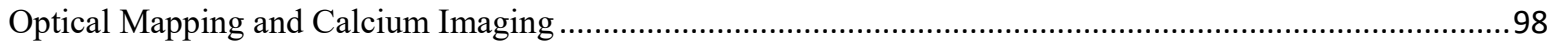


Micro-electrode Array Recordings...................................................................................................... 99

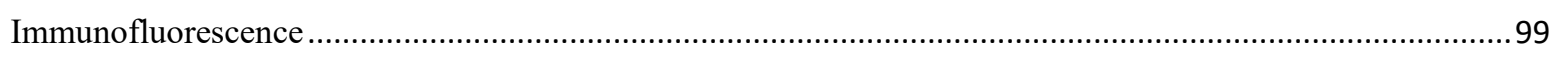

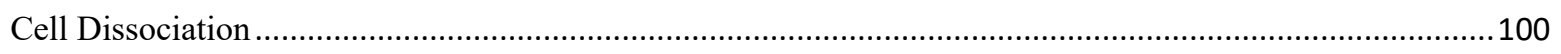

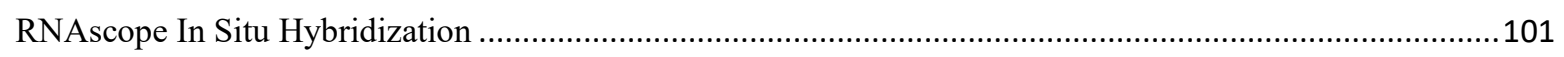

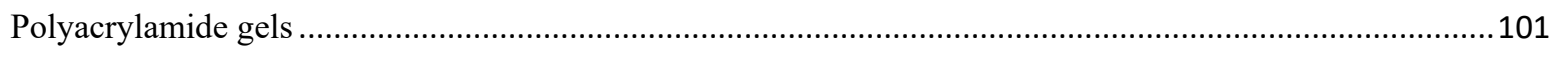

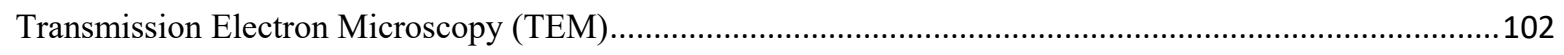

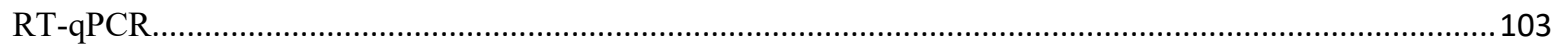

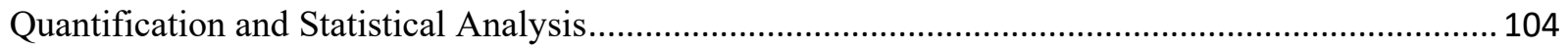

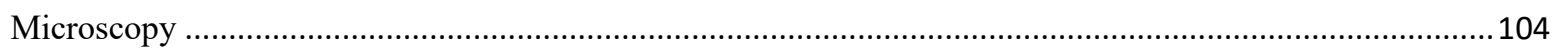

Quantification Area, Surface area, and Volume................................................................................... 105

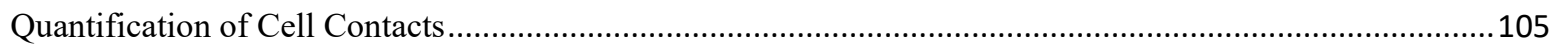

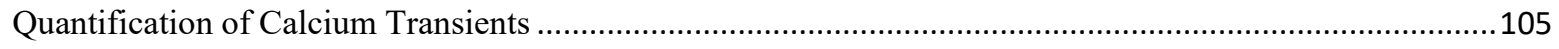

Quantification of N-Cadherin Junctional Intensity ...............................................................................106

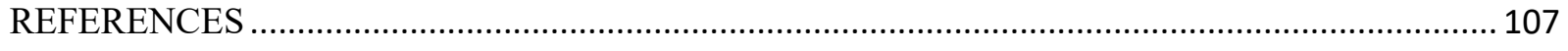

CHAPTER 4: CONCLUSION AND FURTURE DIRECTIONS ...................................... 116

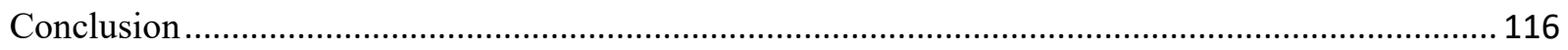

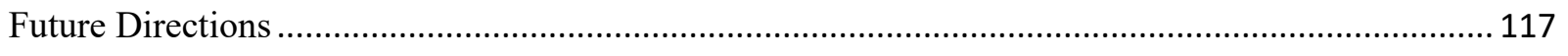

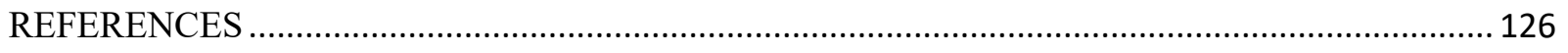

APPENDIX: MICROINJECTION-BASED SYSTEM FOR IN

VIVO IMPLANTATION OF EMBRYONIC CARDIOMYOCYTES

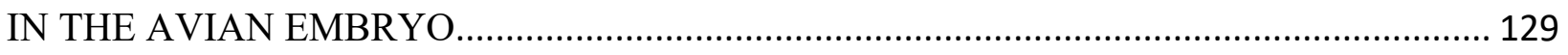

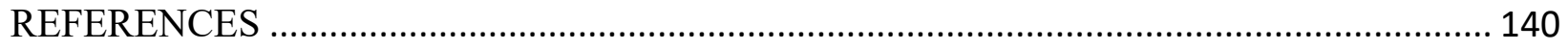




\section{LIST OF TABLES}

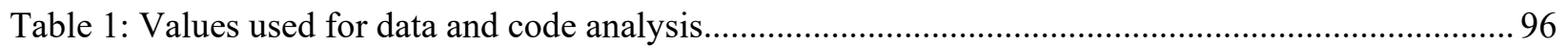

Table 2: List of Materials for transfection and in vivo implantation of embryonic cardiomyocytes in the

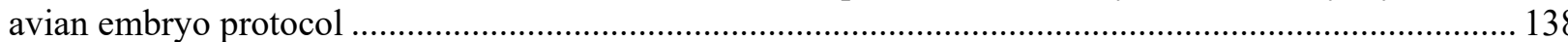




\section{LIST OF FIGURES}

Figure 1: Arrangement of the cardiac conduction system inside a four-chambered heart......................... 5

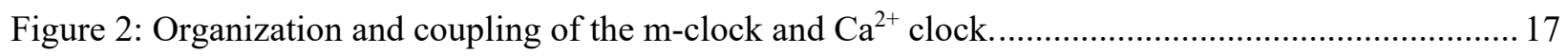

Figure 3: The interplay between genetics, physiology, and cell biology in CPC maturation.................... 20

Figure 4: Organization of the mature cardiac conduction system...................................................... 34

Figure 5. Optical mapping allows for integrative, simultaneous acquisition of

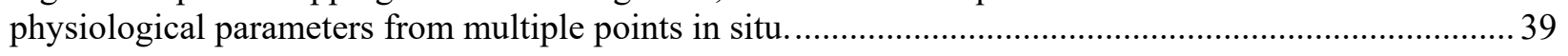

Figure 6. Comparison of voltage-sensitive dyes to small-molecule calcium indicators......................... 43

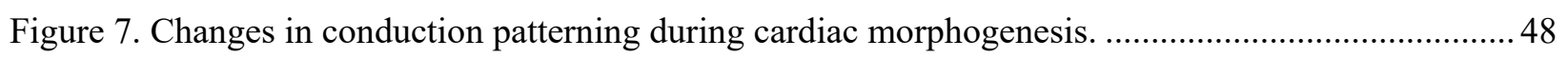

Figure 8. Mosaic analysis for imaging cardiac cell physiology maturation............................................56

Figure 9. Mathematical simulation of electrical interactions in the embryonic heart

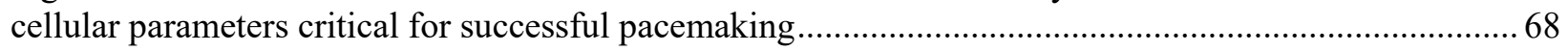

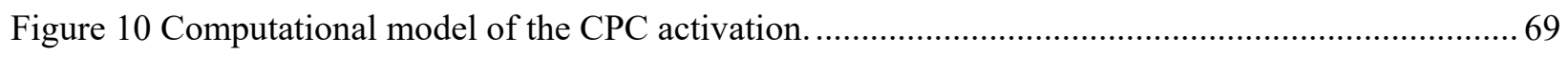

Figure 11. Cell size is differentially regulated between the CPC and WM lineages

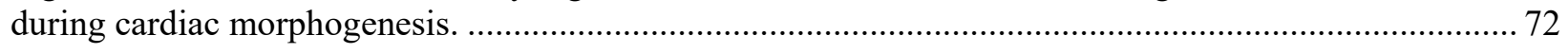

Figure 12. In situ cell size determination....................................................................................... 73

Figure 13. CPCs uniquely lose the capacity to form adherens junctions as they

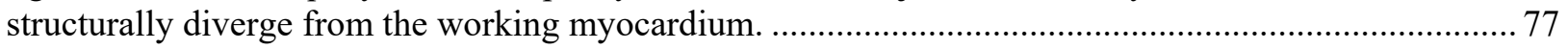

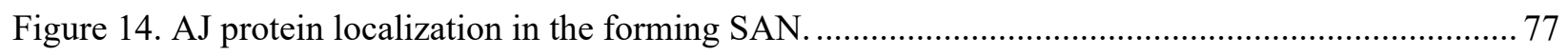

Figure 15. Inhibition of the cardiac AJ converts atrial WM to a CPC-like morphology......................... 80

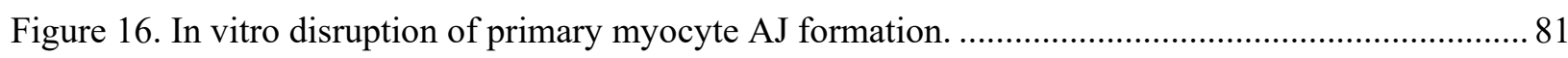

Figure 17. Force-mediated AJ engagement induces a WM-like phenotype in CPCs............................ 83

Figure 18. Increasing CPC size decrease capacity for high-rate electrical oscillation............................. 84

Figure 19. AJ and gap junction proteins colocalize within embryonic CPCs and atrial WM................... 86

Figure 20. Cx40 re-localized to basal cell surface in atrial WM plated on N-cad.................................. 87

Figure 21. Negative Regulator of Cadherin Function, FLRT3, Disrupts CPC AJ Engagement................. 89

Figure 22. FLRT3 Localization and Overexpression.................................................................. 90

Figure 23: Representative images of embryos isolated 24 hours post injection. ................................... 136 


\section{LIST OF ABBREVIATIONS}

AAV

ACP

AJ

aT-cat

AVN

B-cat

CCS

CHD

CPC

Clickseq

CR

ECG

ECM

FN

FACS

GEI

GJ

$\mathrm{HH}$

iPSC

LCR

LED

M-clock
Adeno-Associated Virus

Atrial Conduction Pathway

Adherens Junction

AlphaT-Catenin

Atrioventricular Node

Beta-Catenin

Cardiac Conduction System

Congenital Heart Defects

Cardiac Pacemaker Cells

Click Ligation Next Generation Sequencing

Contractile Region

Echocardiogram

Extracellular Matrix

Fibronectin

Fluorescence Activated Cell Sorting

Genetically Encoded Indicators

Gap Junctions

Hamburger and Hamilton

Induced Pluripotent Stem Cells

Locally Propagating Sub-Sarcolemmal Ca Releases

Light-Emitting Diodes

Membrane Clock 


$\begin{array}{ll}\text { N-Cad } & \text { N-Cadherin } \\ \text { OCT } & \text { Optical Coherence Tomography } \\ \text { OM } & \text { Optical Map } \\ \text { PCA } & \text { Principle Component Analysis } \\ \text { PA } & \text { Polyacrylamide } \\ \text { RyR } & \text { Ryanodidne Receptors } \\ \text { SAN } & \text { Sinoatrial Node } \\ \text { shRNA } & \text { Small Hairpin RNA } \\ \text { SRF } & \text { Serum Response Factor } \\ \text { SR } & \text { Sarcoplasmic Reticulum } \\ \text { TEM } & \text { Transmission Electron Microscopy } \\ \text { VSD } & \text { Voltage-Sensitive Dye } \\ \text { WM } & \text { Working Myocardium }\end{array}$




\section{CHAPTER 1: THE HISTORY OF CARDIAC PACEMAKER CELLS}

\section{The Heart of The Circulatory System}

Blood, waste and nutrient transportation in vertebrates is regulated by a closed circulatory system. In regards to vertebrates, the closed circulatory system can be described in two parts: the lymphatic system and cardiovascular system (Aspelund et al., 2016). The lymphatic system is made of vessels that move lymph from the extracellular space to the circulatory system and lymphatic organs (skin, brain, eyes, and small intestines) (Baluk et al., 2007; Petrova \& Koh, 2018). Lymph is a fluid-like substance that originates from venous leakage. When venous vessels_-like capillaries_-leak interstitial fluid, macromolecules, and immune cells into the extracellular space, these substances are absorbed by the lymphatic vasculature, forming what is known as lymph. This occurs because blood plasma, a proteinrich fluid, extravasates out of blood vessels and must be returned into blood circulation to maintain systemic tissue health. Lymphatic networks are formed in tissue spaces to drain fluid from the extracellular matrices of tissues (Olszewski et al., 1995; Olszewski, 2003)

The flow of lymph is unidirectional. Lymph moves through the lymphatic capillaries, collecting lymphatic vessels, lymph nodes, and lymphatic ducts, respectively before entering back into the cardiovascular system (Wiig \& Swartz, 2012; Zawieja, 2009). The lymphatic system escapes natural gravitational pressures and prevents backflow in order to return lymph back to blood vessels. Vessels of the lymphatic system are similar in composition to blood vessels, consisting of smooth muscle cells and endothelial cells (Bergwerff et al., 1998; dela Paz \& D'Amore, 2009). They are derived from venous vessels, forming after blood circulation is initiated during development (Sabin,1902) (Nicenboim et al., 2015). Growth factor signaling mediates lymphatic vasculature development by initiating lymphatic endothelial cell budding from veins in the cardiovascular system (Carmeliet et al., 1996; Ferrara et al., 
1996; Karkkainen et al., 2004; Aspelund et al., 2016). There is a hierarchy to the lymphatic system, lymphatic capillaries are the smallest vessels that connect to larger lymphatic vasculature (von der Weid \& Zawieja, 2004; Aspelund et al., 2016).

The flow of lymph in lymphatic vessels maintains tissue homeostasis in the body. Tissue homeostasis regulates tissue growth and renewal processes like cell proliferation, cell death, and cell differentiation (Hampton et al., 2015; Aspelund et al., 2016). Homeostasis is a necessary state to maintain proper tissue function by creating an equilibrium between cells and their environment and allowing cells to communicate with their surrounding extracellular matrix. Tissue homeostasis regulates environmental cues that influence transcriptional regulation and growth factor distribution and maintains a healthy tissue microenvironment; for example, in the case of tissue injury, immune cells aid in tissue restoration (Olszewski et al., 1995). Overall, organs benefit from lymphatic drainage of interstitial fluid, however, organs — like the heart—do not have internal lymphatic vessels as seen in the skin, brain, eyes, and small intestine. From an individualistic point of view, the heart is supported by blood vessels from the cardiovascular system that aid in its tissue-specific fluid drainage (Sherwood, 2007).

The cardiovascular system is comprised of multiple blood vessels that move blood towards and away from the heart via veins and arteries, respectively. The primary function for this system is to deliver oxygenated blood to various tissue and organ systems in the body while simultaneously moving deoxygenated blood into the lungs, where oxygen molecules are packaged into the blood for redistribution throughout the organism. The cardiovascular system consists of a highway of arterial and venous vessels networking to move blood. Arterial vessels branch off from the aorta, the largest artery. Arteries are smaller than veins, yet they have a thicker vessel wall to withstand biophysical forces like sheer stress. Their thick vessel walls are made of endothelial and smooth muscle cells that are transcriptionally distinguishable from endothelial and smooth muscle cells in veins. Venous vessels have a thin endothelial cell wall lined with smooth muscle; these vessels are larger in diameter than arteries (Bloom and Fawcett 1994; Gartner and Hiatt 1994; dela Paz \& D'Amore, 2009). 
In addition to blood vessels, the heart is also an element of the cardiovascular system. The heart is the pump that drives multiple functions of a closed circulatory system: delivering nutrient-rich blood to various tissues in the organism and establishing pressure gradients that remove metabolic wastes (Sherwood, 2007; (Verhoeff \& Mitchell, 2017). Dating back thousands of years, the heart's central role in maintaining life inspired both the anatomical and philosophical investigation into how the body worked. Early philosophers and physicians originally set out to find the source of the human soul and mind. Their curiosity led to the discovery of a muscular organ placed at the center of the chest cavity above the lungs (Celesia, 2012; Mavrodi \& Paraskevas, 2014) . Medical pioneers, such as Hippocrates and Aristotle noted that the veins and arteries of the cardiovascular system connected the entire body to the heart. Their understanding of the heart was dependent on animal dissections and thus human anatomy of the heart was not well-documented until the fourth century in Egypt by Praxagoras of Cos and Herophilus. They documented the differences between veins and arteries (Bay \& Bay, 2010; Mavrodi \& Paraskevas, 2014). Galen was the first anatomist to document the muscular integrity of the heart, noting the muscular thickness of the right versus left side. He also described the direction of cardiac muscle fibers as straight, sectional, and oblique (Sternback, 2001; Galen, 2004). In the days of Galen, the heart was defined as a three-chambered organ. Scientists and philosophers thought the heart consisted of three ventricles, having mistaken the atria for one ventricle. It was not until the 1500 s that the heart was scientifically proven to be a four-chambered organ system by Berengario da Carpi. Thus, scientists came to the conclusion that the heart was made of two atria and two ventricles, each separated by a septum (Van Praagh \& Van Praagh, 1983; Mavrodi \& Paraskevas, 2014).

Uniform muscle contractions of the atria and ventricles drive the tightly regulated process of distributing blood throughout an organism. The left and right sides of the heart are separated by septa, which prevents the merging of deoxygenated and oxygenated blood, and are functionally distinct. Each side consists of one atria and one ventricle. The left atrium and left ventricle pump oxygenated blood from the lungs to various organs and tissues throughout the body (Bestetti, 2014). In contrast, the right 
atrium and right ventricle pump deoxygenated blood into the lungs for oxygen redistribution. Oxygenrich and oxygen-deficient blood move from one chamber of the heart to another through muscle contractions. The atria and ventricles are comprised of many muscle cells with the individual ability to contract. Muscle cells in each chamber of the heart work together to contract the entire chamber, atrium or ventricle, pushing blood out. For instance, in the cardiac cycle, after blood has filled the heart chambers, the atria are the first to contract, pushing blood into the ventricles. This is termed "atrial systole" and "ventricular diastole" because the atrial muscles are contracting and the ventricular muscles are relaxed. This is followed by atrial diastole and ventricular systole; this consists of the atrial muscles relaxing and the ventricular muscles contracting to move blood out of the heart. This is a continuous cycle required to replenish blood and to deliver oxygen- and nutrient-rich blood throughout the entire body. The proper sequence and timing of atrial and ventricular muscle contractions is regulated by electrical signaling in the heart (Alpert et al., 1979; Anderson et al., 2014; Kaul, 1986; Klasnja, 2013; Tan, 1987).

\section{The Anatomy and Function of the Cardiac Conduction System}

The cardiac conduction system (CCS) consists of a network of specialized cells and tissues that control electrical propagation in the heart. Organized conduction through the heart is necessary for proper contraction and blood flow. There are five major components of the CCS: the sinoatrial node (SAN), atrioventricular node (AVN), His bundle, bundle branches, and Purkinje fibers (Figure 1). These components have distinct morphological and functional differences that separate them from the surrounding contractile muscle cells of the atria and ventricles (Park, 2011; Kennedy et al., 2016). The anatomy, morphology, and molecular profiles of each CCS component have been well studied throughout history to present day. The SAN initiates electrical propagation in the heart while the AVN slows

electrical conduction and transfers electrical signal from the atria to the ventricular conduction system. The His bundle moves the electrical signal toward the center of the heart and into the ventricles through the bundle branches and Purkinje Fibers (Keith and Flack, 1907; Tawara, 2000; James, 2002; Akiyama, 2010; Anderson et al., 2009 van Weerd \& Christoffels, 2016). 
The SAN is adjacent to the right atrium on the posterior side. There are multiple anatomical landmarks for locating the SAN, which can be useful due to anatomy variations between different species. Overall, the SAN can be found between the heart's epicardial and endocardial layers, where the right atrial wall meets the superior vena cava. The SAN consists of a head, mid-body, and tail that wrap around the back of the right atrium, similar in shape to a banana (Keith and Flack 1907; Sanchez-Quintana et al., 2005; Ho \& Sanchez-Quintana, 2016). The regional differences of the SAN will be discussed in further details in the subsequent text. Cardiomyocytes in the SAN are termed cardiac pacemaker cells (CPCs); these cells have fewer striations and are smaller in cell size, with an ovoid morphology, when compared to the surrounding cardiomyocytes in the atria.

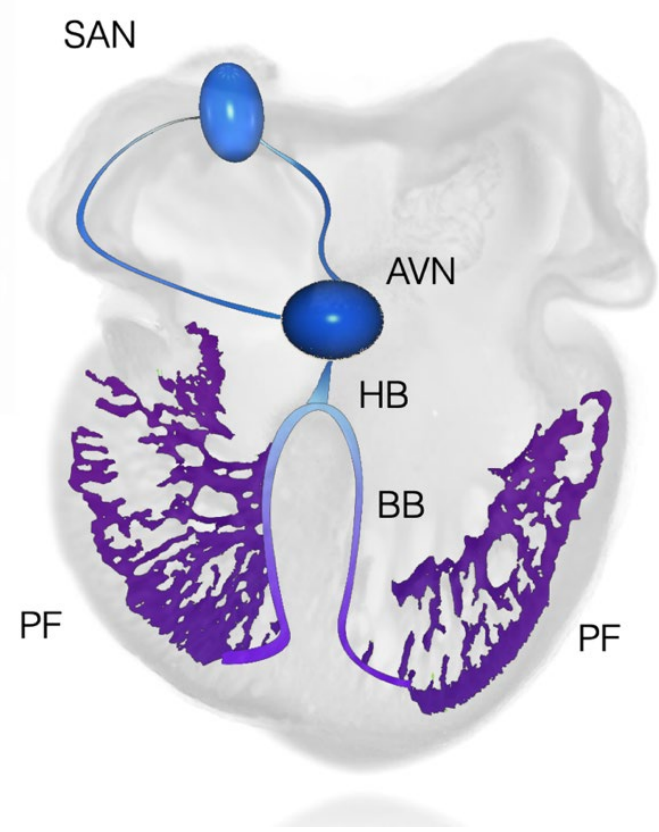

Figure 1: Arrangement of the cardiac conduction system inside a four-chambered heart.

A Schematic of a four-chambered heart indicating the relative positions of the subcomponents of the cardiac conduction system. SAN indicates sinoatrial node, AVN indicates atrioventricular node, HB indicates His bundle, $\mathrm{BB}$ indicates bundle branches, and PF indicates Purkinje-fibers.

CPCs have been described as "spider" shaped cells as well (Masson-Pevet et al., 1979; Woods et al., 1982; Wu et al., 2001). Also, to be discussed in greater detail below, the defined cell junction present amongst most cardiomyocytes, the intercalated discs, are not well-defined in cardiac pacemaker cells. 
CPCs contribute to the unique molecular signatures found in the SAN. There are distinct gene expression patterns in the SAN such as the HCN channel. The most common HCN channel gene expressed in the developing and adult SAN is HCN4 (Liang et al., 2013; Vedantham et al., 2015; Kozasa et al., 2018). In addition to HCN4, many other genes are enriched in the SAN throughout the course of development and maturation: ACNA1G (CAV3.1), CACNA1H, SHOX2, ISL1, BMP4, TBX3, TBX18, CX30.2, COL3, SMOC2, CACNA1D (CAV1.3). Additionally, the SAN is generally characterized as a SCN5A-, NKX2.5-, PITX2-, NPPA- and CX40-depleted region (Mori et al., 2006; Christoffels et al., 2006; Hoogaars et al., 2007; Mommersteeg et al., 2007; Blaschke et al., 2007; Wiese et al., 2009; Espinoza-Lewis et al., 2009; Vedantham et al., 2015). The complexity of the transcriptional profile of the SAN increases when investigating individual species.

Following the path of conduction, the next CCS element, below the SAN, is the AVN. The AVN is located between the two atrial chambers and ventricular chambers, positioned toward the right atrial side of the heart. Tawara described what we call the AVN as a series of muscle fibers originating on the atrial side of the fibrous av septum and penetrating through to the muscular ventricular septum. In general, AVN cardiomyocytes are distinct from the atrial working myocardium in that they are less dense, have fewer and poorly arranged myofibrils, and possess fewer intercalated discs. In contrast to neighboring atrial cells, the myocytes of the AVN stain pale pink with a trichrome stain (Tawara, 2000; Anderson et al., 2009; Akiyama, 2010). The AVN includes multiple compartments, arranged into a triangular region such as the compact AVN, lower nodal bundle, penetrating bundle, central fibrous bundle, and right and left inferior extensions. These structures differ in their relative abundance of intercalated discs and gap junctions. Myocytes in the AVN are typically characterized as low nodal cells or midnodal cells. Midnodal cells are located in the compact AVN, these cells are tightly packed with little connective tissue surrounding them. Low nodal cells are in contact with both midnodal cells and transitional cells, described below. Low nodal cells are elongated compared to other AV nodal cells, however they are smaller than atrial myocytes. Towards the posterior end of the AVN there are a 
populations of transitional cells, which are separated from the surrounding atria by connective tissue (Anderson et al., 1974; Markowitz \& Lerman, 2018). Spanning development and maturation, the AVN molecular profile is described as a(n) HCN, KCNE1, CACNA2D2, CACNA1G, CPNE5, TBX5-, TBX3, and RGS6-positive region with varying expression patterns dependent on AV node compartments (Goodyer et al., 2019).

Next, in the conduction pathway is the His bundle and bundle branches. The His bundle is connected to two bundle branches and all three components are anatomically located in the interventricular septum space. The His bundle connects to the AVN at the posterior end of the interatrial septum, descends into the interventricular septum space and then begins to branch into the right and left sides of the heart. The left bundle branch is notably larger than the right bundle branch. These anatomical differences in the bundle branches are slightly expected given the fact that the left side of the heart is larger than the right. The His bundle, left bundle branch, and right bundle branch consist of cardiomyocytes and extracellular matrix (ECM). Cardiomyocytes of the His bundle and left bundle branch are arranged parallel to each other on the longitudinal plane. These myocytes are elongated and are ensheathed by collagen. The cellular morphology of the right bundle branch is similar to the His bundle and left bundle branch, however, there is more morphological variety between these cells.

Overall, myocytes in the His bundle and bundle branches are smaller and fewer in myofibrils when compared to the atrial and ventricular working myocardium. Also, these myocardial cells are short and depicted as having an oval shape (Tawara, 2009; Anderson et al., 2009; Akiyama, 2010; Sharma et al., 2019). Similar to the AVN, the His bundle and bundle branches are generally characterized as KCNE1, TBX3, TBX5, and RGS6 enriched regions. As well as SCN5A, ETV1, IRX3, CX40 enriched regions and CX43 negative regions (Goodyer et al., 2019).

Historically, the CCS as a whole is enriched for the genes HCN4, GJC1, CACNA2D2, IGFBP5, CPNE5, and NTM (Burnicka-Turek et al., 2020; Goodyer et al., 2019; Munshi, 2012). There are multiple differences in molecular profiles of individual CCS components as described above. The specific 
electrophysiological properties of the CCS components will be discussed in greater detail below. The CCS can be described as having two parts: the ventricular and the atrial conduction systems. The ventricular conduction system is the most studied and well-described conduction system, often referred to as the His-Purkinje network. The ventricular conduction system consists of a network of His bundle, bundle branches, and Purkinje fibers. As mentioned above, these components are located in the ventricular chambers and their primary function is to facilitate blood flow in and out of the ventricles by signaling the ventricular cardiac muscles to contract. The ventricular conduction system regulates fast electrical impulses from the atria to the walls of the ventricle. This is done when the AV node delivers electrical currents to the His bundle. As stated previously, the His bundle branches into the left and right ventricles through bundle branches, which receive electrical currents from the His bundle. This rapid electrical propagation terminates at the Purkinje fibers before repeating in the next heartbeat. Ventricular conduction cells depolarize the ventricles once the ventricles are filled with blood, initiating ventricular systole. The ventricles then contract to push blood out of the heart through the pulmonary artery and aorta (Catanzariti et al., 2013; Goodyer \& Wu, 2018; Haissaguerre et al., 2016; Huang et al., 2017; Midgett et al., 2017).

The atrial conduction system consists of the sinoatrial node and AVN, which are characterized as slow conducting nodes of the atria. In addition to the slow conducting nodes, internodal pathways have been described in various atrial conduction system models. Internodal conduction pathways in the atrial electrical system is still a controversial topic (Anderson \& Ho, 1998). Nonetheless, electrical currents travel from the SAN through the internodal pathways to the AVN. The electrical signal is slowed in the AVN before reaching the ventricles, giving the ventricles time to fill with blood and complete diastole. Slowed conduction in the AVN also ensures proper atrial contraction/atrial systole to passage blood into the ventricle chambers. This conduction propagation process is repeated at the beginning of every heartbeat and is necessary to maintain a steady heart rate. The SAN of the atrial conduction system sets the rhythm of the heart by firing spontaneous action potentials to initiate electrical propagation in all four 
heart chambers. Thus, the SAN is the pacemaker of the conduction system (James, 2002; Silverman, 2006; Akiyama, 2010; Markowitz \& Lerman, 2018).

The final CCS component, yet to be discussed are the Purkinje Fibers. This CCS feature is located in the two ventricular chambers of the heart. Purkinje fibers branch out from the left and right bundle branches into the subendocardial space of the ventricles. Unlike any other component of the CCS Purkinje fibers have an abundance of organized myofibrils. In addition to their contractile apparatus organization, Purkinje Fibers are larger than ventricular myocytes. These cells stain a darker pink compared to their surrounding working myocardium according to Hematoxylin and Eosin data, demonstrating their dense cellular structure. Although, Purkinje fibers are located in the ventricular chambers they are spatially isolated from the working myocardium (Keith and Flack, 1907; Hyer et al., 1999; Sedmera, 2014; Ho et al., 2002). Similar to other CCS counterparts, Purkinje fibers have a distinct transcriptional profile as well. Differing from its CCS neighbors Purkinje fibers are generally described as a CX40, CX43, SCN5A, ETV1, SEMA3A, and NKX2.5 enriched track of cells (Burnicka-Turek et al., 2020; Maass et al., 2015).

\section{The SAN is a Heterogenous Tissue}

The mechanism for synchronous muscle contraction of all four heart chambers was unknown until the 20th century. It was well understood that the heart contained rhythmic properties, but early physiologists could not distinguish whether these properties were neurogenic or myogenic in origin. Until the 1800 s, scientists concluded that the heartbeat originated from ganglion cells like those found in skeletal muscle. Scientists hypothesized that since heart striations are similar to those found in skeletal muscles, contractions must be initiated in similar mechanisms. This mechanism was termed the neurogenic theory and was widely accepted in the cardiology field. In 1883 scientists began to question this theory after Dr. Walter Gaskell showed that the muscle contractions in the heart are independent of the nervous system. Gaskell was able to understand the impulse pathway of the heart, describing it as originating from the sinus venosus, and spreading downwards towards the atria and ventricles, 
respectively. This led to the myogenic theory, which states that muscle cells within the heart itself stimulate cardiac contractions. After this discovery scientists began to experiment in the heart to uncover the source of cardiac contractions (Fenton, 1908).

The knowledge gap in the field, relating to where cardiac contractions originate led to the invention of the electrocardiogram by William Einthoven in 1895. The evaluation of the electrophysiology of the heart was not possible until Einthoven's electrocardiogram. However, it was Dr. Sunao Tawara's research on the atrioventricular conduction pathway that enabled physiologists to interpret electrocardiogram data (Tawara, 2000; Akiyama, 2010). Tawara research was published in 1906 and concluded that the AVN, His Bundle, bundle branches, and Purkinje fibers were all connected to conduct electrical currents from the atria to the ventricle. Shortly after, in 1907, Arthur Keith and Martin Flack discovered the anatomical location of the pacemaker of the heart. Through a variety of mammalian heart dissection Keith and Flack discovered a population of striated cells surrounded by dense connective tissue, that was similar in structure to the AVN, and they initially termed this region of the heart the sinoauricular node (Keith and Flack, 1907; Akiyama, 2010).

In all mammal models that Keith and Flack investigated, the sino-auricular node was located at the junction of the superior vena cava and atrial muscular wall. Keith and Flack postulated that electrical propagation in the heart is initiated in the pacemaker region also referred to as the SAN. In 1909, Thomas Lewis confirmed that the birthplace of electrical excitation in the heart is the SAN after a series of experiments. He applied an ectopic electrical stimulus to various regions including the SAN to confirm electrical wavelength curves previously recorded at the start of a cardiac impulse. The wavelength curves recorded by the SAN had similar properties to those seen at the beginning of the heartbeat. The SAN was the last cardiac conduction system component to be discovered, and since then the SAN has been largely defined by the structural complexity first noted by Keith and Flack (Keith and Flack, 1907; Akiyama, 2010). 
More specifically, the SAN is a heterogenous tissue composed of multiple cell types and extracellular matrix, each critical for proper pacing of the heart. The SAN center is composed of CPCs, historically termed nodal or "P" cells, that pace the heart. These cells control the heart's electrical impulse by cell autonomously initiating action potentials. This electrophysiological process will be discussed in further details in future chapters of this dissertation. CPCs are distinguishable from other cells types in the SAN as they are 'empty' cardiomyocytes compared to neighboring atrial cells, because they have few and unorganized myofilaments. As will be investigated further in later chapters, microscopy studies conducted over the last century have determined that CPCs are cells that are round and small in cellular morphology. Also, these cells have fewer junctions, which result in poor coupling between CPCs (Woods et al., 1976; Bleeker et al., 1980; Masson-Pevet et al., 1984; Opthof et al., 1986; Opthof et al., 1987; Boyett et al., 2000; Miyamoto et al., 2002).

Moving towards the periphery of the SAN, transitional cells separate CPCs from atrial cardiomyocytes. Transitional cells are neither atrial myocardium or CPC myocardium. These cells represent the site of atrial myocardium and CPC intermixing. Transitional cells are characterized from other SAN cell types as having less fibrous connective tissue between myocytes, poor cell junctional coupling, and poorly aligned muscle fibers (Trautwein and Uchizono, 1963; Bleeker et al., 1980; MassonPevet et al., 1984; Opthof et al., 1986; Opthof et al., 1987; Woods et al., 1976; Boyett et al., 2000; Miyamoto et al., 2002).

Along with various myocardial cell types the SAN consists of a host of non-muscle cell populations. Immunohistochemistry of these cells do not positively correlate with myocardium markers. The central molecular characteristic of this cell type is that they are WT1 positive. The significance of the epicardial marker WT1 and the origin of this non-muscle mesenchymal cell population will be discussed in further detail in a later chapter. Lastly, CPCs are embedded in collagen-rich extracellular matrix. It is unclear which SAN cell type deposits the extracellular matrix, but in the absence of the non-muscle mesenchymal cell population, interstitial collagen is lost. The SAN extracellular matrix is historically 
described as a fibrous connective tissue (Bressan et al., 2018). This unique environmental niche maintains the SAN tissue structure necessary for proper CPC function.

In summary, the SAN is an isolated microenvironment, the source of electrical current, and located in the back of the right atrium. It is a common belief that CPCs are insulated from a hyperpolarized atrial working myocardium through poor gap junction coupling. There is a small source of CPCs compared to the total mass of the working myocardium. It is estimated that CPCs compose less than $.0005 \%$ of cardiomyocytes in the heart with approximately $2-4$ billion cardiomyocytes in the working myocardium. A recurring question in the field is how does this small electric current source depolarize a much larger working myocardium (Adler \& Costabel, 1975; Joyner \& van Capelle, 1986; Vedantham, 2015)?

It has been widely described that CPCs must maintain a balanced source-sink relationship with the working myocardium. This is an electrogenic principle related to the barriers that exist when a small group of excitable cells need to induce the depolarization of a much larger volume of tissue. This is a fairly unique biological principle that is critical for conduction through the heart and is most relevant to pacemaking in that theoretical evaluation suggests that the surrounding atria is larger and therefore is capable of generating local currents that should suppress electrical drive from the SAN. Among the first to describe this phenomenon in the SAN were Joyner and van Capelle (1986), who conducted a series of computational experiments using membrane models of the SAN and atrium. Their stimulations showed that when the atria and SAN membranes are equal the SAN fails to drive electrical propagation through the atria. Only when the SAN membrane area is increased to $1.2 \mathrm{x}$ higher than the atria membrane is successful pacing and electrical propagation achieved. Then, when the atrial membrane area is higher than the SAN membrane area pacing and propagation is lost. Thus, the SAN should theoretically not be able to drive a larger atrium when the two chambers are electrically linked via a uniform conductive interaction (Joyner \& van Capelle, 1986; Kleber, 2011; Unudurthi et al., 2014; Issa, 2019). 
Since the initial work by Joyner and Capelle, it has become clear that stable source-sink relationship between the SAN and the working myocardium depends on multiple factors. First, "source" is defined as the current that is cell autonomously generated in the SAN by CPCs. "Sink" is defined as the

amount of current from the "source" needed to excite/depolarize quiescent cardiomyocytes in the working myocardium. This fragile relationship is dependent on maintaining specific cellular interactions, tissue integrity, and membrane electrophysiology in both the SAN and working myocardium. For instance, it is characteristic of the working myocardium to form intercalated discs, have less fibrous tissue, and a resting membrane potential. In contrast, SAN cells are poorly coupled and embedded in fibrous tissue which separates them from the working myocardium. These biological parameters in the SAN are necessary to electrically isolate CPCs. Thus, voltage changes that occur while a CPC depolarizes remain local to those cells and are not influenced by the hyperpolarizing load of the working myocardium (Winfree, 1967; Bleeker et al., 1980; Joyner \& van Capelle, 1986; Bottani, 1995 Kleber, 2011; Unudurthi et al., 2014; Issa, 2019).

\section{The Physiology of CPCs}

Automaticity in CPCs is defined as action potentials generated in a cell autonomous fashion. $\mathrm{CPCs}$ are able to generate spontaneous action potentials without an upstream stimulus to begin the excitation cycle (Keith and Flack, 1907 ;Bouman \& Jongsma, 1986; Kohajda et al., 2020). How CPCs generate action potentials is well-studied. To understand how CPCs fire action potentials at the onset of the cardiac cycle, we need to first examine the interior of these pacing cells and discuss the events that regulate slow diastolic depolarization and action potential upstroke. Slow diastolic depolarization is the key physiological characteristic of CPCs. It is defined as the activation of inward ion currents which result in depolarization of the membrane and make the membrane potential more positive (Kohajda et al., 2020; Mangoni \& Nargeot, 2008). This activity can be seen on an isopotential map.

There are three phases of the CPC action potential that illustrate when these ion electrochemical gradients in the sarcolemma open and close. First, during phase 4, the "funny" channel $I_{f}$ ) initiates 
spontaneous slow depolarization of the $\mathrm{CPC}$ membrane by the movement of $\mathrm{Na}+$ and $\mathrm{K}+$ ions to achieve threshold membrane potential. The hyperpolarized $\mathrm{I}_{\mathrm{f}}$ facilitates the influx of these ions into the cell, given this channel's "leaky" nature. After the membrane reaches threshold potential of $\sim-40 \mathrm{mV}$, diastolic depolarization continues into phase 0 . The phase of diastolic depolarization after threshold potential is met is also termed action potential upstroke. During phase 0 , the slow influx of $\mathrm{Ca}^{2+}$ ions increases through L-type calcium channels (iCa,L), that open during this time. Following phase 0 is phase 3 of the action potential in which delayed rectifier $\mathrm{K}+$ channels $(\mathrm{iK})$ open; this process is called repolarization when the negativity of the membrane potential increases. There are two types of $\mathrm{iK}$ currents present in CPCs: slow delayed rectifier $\mathrm{K}+$ channels (iKs) and rapid delayed rectifier $\mathrm{K}+$ channels $(\mathrm{iKr})$.

Simultaneously, when $\mathrm{iK}$ channels open iCa, T channels close, decreasing the transport of $\mathrm{Ca}^{2+}$ ions into the cell. Multiple studies have shown that CPC excitation is dependent on these ion channels. When one or more of these channels is inhibited, pacemaking function in CPCs decreases. For instance, when $\mathrm{iCa}, \mathrm{L}$ channels are blocked in rabbit spontaneous action potentials in CPCs are lost. When an $\mathrm{iKr}$ blocker is introduced to rabbit CPCs, action potential duration increases. Lastly, previous experiments have shown that when $\mathrm{I}_{\mathrm{f}}$ channels are inhibited, CPC function is not completely lost. When $\mathrm{I}_{\mathrm{f}}$ blockers such as, Cs+, zatebradine, and ZD-7288 are introduced into the rabbit SAN, decreased pacemaker spontaneous rate and upstroke were observed. These experiments have led to a controversial role for $\mathrm{I}_{\mathrm{f}}$ in pacemaking function. Since, inhibiting $\mathrm{I}_{\mathrm{f}}$ does not abolish CPC spontaneous action potential generation, some believe that it has a minor role in the CPC excitability cycle. While others, argue that $\mathrm{I}_{\mathrm{f}}$ channels are singularly responsible for CPC action potentials (Goethals et al., 1993; Lakatta et al., 2010; Maltsev \& Lakatta, 2008; Mangoni \& Nargeot, 2008).

All of the above occur at the cell surface and have thus become known as the pacemaker membrane clock, or M clock.

However, pacemaker cells also contain a separate intracellular ionic cycling system that supports their electrical automaticity. This system is based in the sarcoplasmic reticulum (SR). 
The SR is a membrane-bound organelle that is distinct to muscle cells. This organelle regulates intracellular calcium ion storage and, in return, transmits local cellular electrical currents. The SR transports subcellular calcium ions through its localized system of pumps and receptors. The main drivers of intracellular calcium release in the SR are ryanodine receptors (RyRs), which are embedded in the SR membrane. Then, calcium is moved into the SR through calcium adenosine triphosphatase pumps (Serca)(Annunziato, 2013; Lyashkov et al., 2018; Monfredi et al., 2013; Vinogradova et al., 2004).

In most cardiomyocytes, RyR release of calcium into the cytoplasm and sarcoplasmic/endoplasmic reticulum calcium ATPase's (SERCA) clearance back into the SR are the consequence of activation and drive the contractile cycle of the cell. In pacemaker cells, $\mathrm{Ca}^{2+}$ homeostasis, through RYR, SERCA, and the $\mathrm{Na}^{+} / \mathrm{Ca}^{2+}$ exchanger (NCX), plays a vital role in CPCs' pacemaking function and contributes to slow diastolic depolarization. In pacemaker cells, reloading of the $\mathrm{SR}$ with $\mathrm{Ca}^{2+}$ by SERCA, triggers RYR opening. In turn, this releases $\mathrm{Ca}^{2+}$ into the cytoplasm. Increased cytoplasmic $\mathrm{Ca}^{2+}$, in turn, activates NCX, which pumps one $\mathrm{Ca}^{2+}$ ion out of the cell for three $\mathrm{Na}^{+}$ions.

NCX is a transmembrane electrochemical gradient that was first described by Reuter and Seitz. NCX functions as an electrogenic exchanger that brings into the cell three $\mathrm{Na}^{+}$cations for one $\mathrm{Ca}^{2+}$ cation release outside the cell, making the exchange ratio $3: 1$. This exchange has the ability to depolarize or repolarize muscle cells by generating an inward or outward current respectively (Annunziato, 2013; Reuter \& Seitz, 1968). The exchanger is activated by protein phosphorylation regulatory elements such as PKC and PKA activators. Thus, protein dephosphorylation is an inhibitory factor for NCX. NCX was first discovered in CPCs in 1984 by Brown et al. They claimed that NCX was a slow component of the inward current during the CPC action potential cycle. The inward current of NCX is dependent on the extracellular release of $\mathrm{Ca}^{2+}$ (Brown et al., 1984). This experimental conclusion was an important piece of information to explain how the two oscillating clocks are coupled. There are multiple isoforms of NCX that can be found in the brain such as NCX1, NCX2, and NCX3 (Lu et al., 2002). There is only one isoform of NCX found in the heart, which is NCX1. When NCX1 is knocked down or ablated in 
cardiomyocytes, multiple diseases and abnormalities arise, thus, NCX1 is necessary for proper cardiac rhythm (Hermann, 2013). There have been numerous models of NCX's role in CPC automaticity over the years since the early $90 \mathrm{~s}$.

In 2000, Huser et al. provided experimental evidence that supported NCX's role on locally propagating sub-sarcolemmal $\mathrm{Ca}^{2+}$ releases (LCRs). LCRs are spontaneous, independent of the M-clock, and are regulated by RyRs in the SR. Huser et al. suggested that during diastolic depolarization when $\mathrm{iCa}, \mathrm{T}$ channels are active, subsarcolemmal $\mathrm{Ca} 2+$ release is initiated, which then triggers $\mathrm{NCX}$ inward currents. Activation of NCX by LCRs was supported by experimental findings of Yaniv et al. They showed that when CPCs were introduced to caffeine, $\mathrm{Ca}^{2+}$ release was initiated, which increased NCX activity. The NCX inward current is important for CPC function because it depolarizes the cell membrane allowing the action potential to reach threshold. It has been predicted that NCX activates the latter iCa,L channels. When NCX is inhibited by disrupting the $\mathrm{Na}^{+}$and $\mathrm{Ca}^{2+}$ cation exchange, the loss of a membrane inward current results in loss of CPC function (Huser et al., 2000).

There are two phases to the $\mathrm{Ca}^{2+}$ clock system: tick and reset, or ignition and refilling the SR with $\mathrm{Ca}^{2+}$, respectively. During the ignition/tick phase, after NCX is activated by LCRs, action potentials are triggered. Once action potentials are activated, this results in a CICR response, meaning that $\mathrm{Ca}^{2+}$ is released from the SR. Induced $\mathrm{Ca}^{2+}$ release depletes the SR during the action potential, so cycle and refilling is necessary to repeat this cycle. These LCRs occur during late diastolic depolarization. During refilling/reset, cytosolic $\mathrm{Ca}^{2+}$ transient activity signals CaMKII-activity and inactivation of $\mathrm{iCa}, \mathrm{L}$ channels. CaMKII-activity is important because this leads to reactivation of $\mathrm{Ca}^{2+}$ channels. This increases $\mathrm{Ca}^{2+}$ influx to load $\mathrm{Ca}^{2+}$ into the SR through RyRs (Figure 2). Refilling is a longer phase than the ignition phase and occurs during repolarization of the action potential cycle. The action potential cycle is dependent on how fast the SR can cycle through the ignition and refilling phases (Chiesi et al., 1981; Maltsev \& Lakatta, 2008; Vinogradova et al., 2000). 


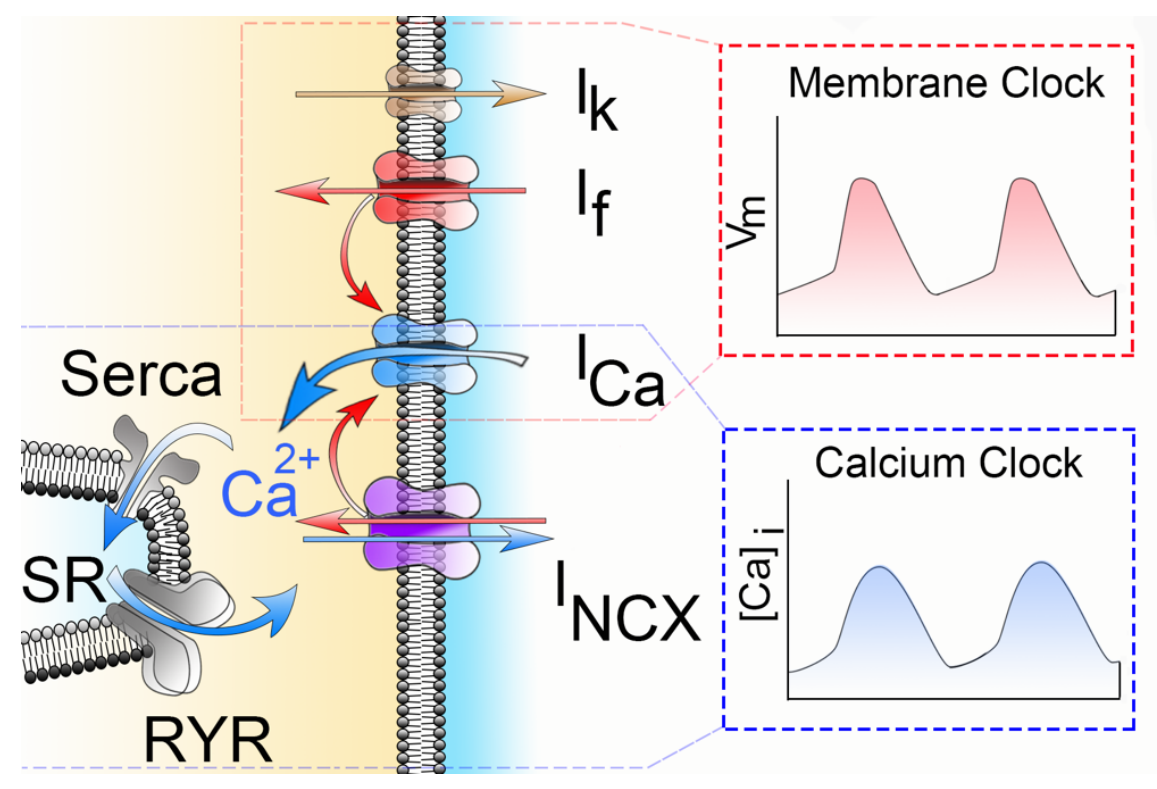

Figure 2: Organization and coupling of the m-clock and $\mathrm{Ca}^{2+}$ clock.

Schematic of the m-clock and $\mathrm{Ca}^{2+}$ clock inside cardiac pacemaker cells. These clock systems make up CPC physiology and are coupled through interactions with the $\mathrm{I}_{\mathrm{ca}}$ channel. Outlined in red indicates components of the membrane clock system and outlined in blue indicates the components of the $\mathrm{Ca}^{2+}$ clock system. These two coupled clock systems regulate membrane potentials in CPCs.

You may have noticed there is a vast system of pumps, channels, and receptors that contribute to pacemaker cell automaticity. Current research in the field is largely focused on understanding how these systems, particularly the $\mathrm{M}$ clock and $\mathrm{Ca}^{2+}$ clock interact with each other to drive stable oscillation

(Figure 2). In general, it is currently believed that these systems entrain one another so that they operate at the same frequency. This creates a robust system of synchronized membrane and intercellular ionic cycling. Currently, $\mathrm{NCX}$, which is sensitive to intracellular $\mathrm{Ca}^{2+}$ concentration and contributes to slow diastolic depolarization, is thought to play a central role in maintaining communication between the two CPC clocks.

\section{The Transcriptional Landscape of CPCs}

As previously discussed, the function of the sinoatrial node is dependent on a number of physiological pathways and cycles. In addition to CPCs' distinct electrophysiology, there is a specific genetic code that contributes to their development and function. The genetics of CPC development have been studied in a variety of vertebrate model organisms. Thus, the transcriptional landscape of the 
embryonic SAN is well known. A common model of CPC development holds that common cardiac genes responsible for working myocardial characteristics are actively repressed in the SAN resulting in specialized CPCs that resemble embryonic cardiomyocytes. There are multiple transcription factors, ion channels, connexins, calcium interacting proteins, and active signaling pathways whose expression supports this model.

The overall SAN transcription factor program has been well-studied and established in the field. Specifically, Tbx3, Tbx18, Shox2, and Is11 are well known regulators of SAN development. Tbx3 and Tbx18 are a part of the T-box family of transcription factors. The embryonic SAN is Tbx $18+$ but the adult SAN contains little to no Tbx 18 expression. It is unclear what Tbx18 regulates downstream but when Tbx18 is ablated during development, it results in malformation of the "head" region of the SAN. Thus, Tbx18 is required for SAN structure formation, but Tbx18 alone does not appear sufficient for pacemaking specification during cardiac development. Tbx3 is a transcription factor that represses the atrial gene programming by inhibiting Nkx2.5 (among other genes). Unlike Tbx18, Tbx3 is highly expressed in the adult SAN and when it is ablated in the adult SAN, pacemaking function and SAN structure is compromised.

Shox 2 and Is11 are transcription factors expressed in the developing cardiac inflow tract myocardium. This is important because during development the SAN is localized to the inflow tract. Shox2 is required for differentiation of CPCs. Later in development, Shox2 establishes a boundary between the forming right atria by suppressing $\mathrm{Nkx} 2.5$ expression in the $\mathrm{SAN}$ through direct interaction. The SAN is generally characterized as an Nkx2.5 negative/low expressing region in multiple species. Shox 2 further represses the atrial gene programming by inhibiting expression of Nppa and $\mathrm{Cx} 40$ in the SAN. Is1 1 and Shox 2 are dependent on one another and work together upstream of Tbx3 to regulate SAN genes. Is11 is necessary for differentiation of varying heart cell types, including CPCs. Is11 is also necessary for pacemaker cell stability, automaticity, and proliferation. Isl1 is linked to multiple signaling pathways, some of which (such as BMP4) have been noted as important for pacemaker development. 
As mentioned previously, the SAN is a heterogenous microenvironment that is insulated from the working myocardium (WM). Expression and non-expression of specific connexins in the SAN contributes to protection from hyperpolarized atria. There are three dominant connexins present in the heart: $\mathrm{Cx} 40, \mathrm{Cx} 43$, and $\mathrm{Cx} 45$. The atria have been well characterized as $\mathrm{Cx} 40$ and $\mathrm{Cx} 43$ rich regions. Similarly, these connexins are highly expressed during development in the region that will eventually become the SAN. This region is termed the sinus venosus, which forms after atrial chamber formation. When the atria-like connexin expression patterns in the sinus venosus are being established during SAN development, this is called atrialization. This process is termed atrialization of the sinus venosus because the connexin patterning is similar to the atria. However, as the SAN becomes more defined and matures, it diverges from the atrial WM by repressing expression of Cx40 and Cx43 (Mommersteeg et al., 2007). The mature SAN is distinguishable by its enrichment for $\mathrm{Cx} 45$ and $\mathrm{Cx} 30.2$ (van Eif et al., 2018; Vedantham, 2015).

In summary, there is a complex developmental gene program in place during SAN development that is believed to bias cells into the CPC lineage. This program is thought to repress the heart's WM program and favors the activation of genes thought to support functional automaticity and structural insulation from the WM. Importantly, most studies focused on the specification of the pacemaker lineage have thus far focused on the transcription pathway that influence fate. As such, how these cells adapt and respond to the dynamic cellular interactions that occur during cardiac morphogenesis remain poorly understood. Thus, the field currently lacks a robust understanding of the cell biology and cellular mechanics that influence CPC developmental maturation.

\section{The Interplay Between CPC Cell Biology, Genetics, and Physiology}

Currently, the pathways linking the CPC genetic program and the unique cell biological features such as small cell size, poor alignment, diminished junctional organization, and few sarcomeres have not been determined. In general, little is known about how CPCs interpret and respond to even the most basic local stimuli that have been shown to influence working myocardial maturation or disease progression. 
To some extent this knowledge gap is due to a lack of tools to study the cellular adaptions that CPCs may undertake in vivo. In my dissertation work, I have developed new and specific techniques to examine questions related to how CPCs interact with their environment to optimize their electrical activity.

I hypothesized that there are specific genetic, physiological, and cell biological features that work together to generate functioning CPCs (Figure 3). For example, theoretical work presented in Chapter 3 indicates that $\mathrm{CPC}$ small cell size and poor coupling is advantageous for their electrophysiology. Low surface area in CPCs correlates with lower membrane capacitance, which mean less current flux across the membrane is required for depolarization. Similarly, lower coupling conductance means less charge is lost to downstream cells when a CPC is activating. However, how CPC cell size is regulated and how CPCs sense and/or adjust their coupling conductance is not known. Thus, a major focus of my studies has been to relate basic cell biology principles to developmental optimization of CPC patterning.

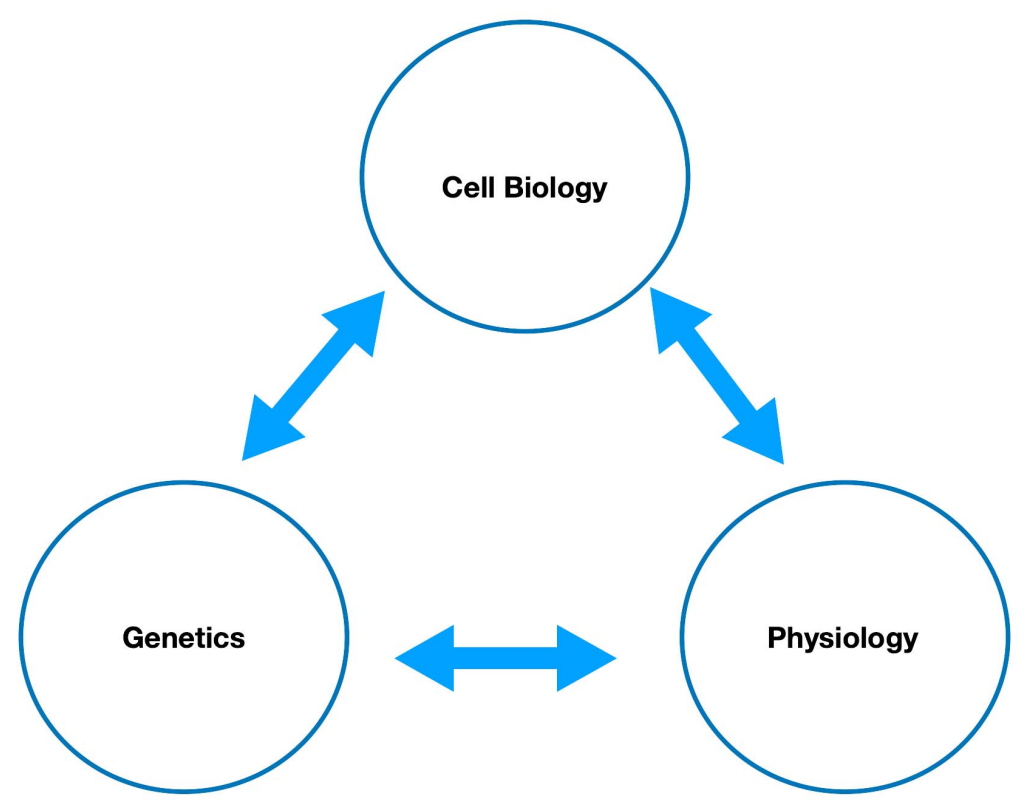

Figure 3: The interplay between genetics, physiology, and cell biology in CPC maturation.

Schematic of the interactions in CPC genetics, physiology, and cell biology. It was hypothesized that during cardiac development the necessary genetic, physiology, and cell biology features of CPCs are built to maintain their unique structure and function.

Developing these tools and exploring the cellular mechanisms that contribute to CPC morphology and function is necessary as SAN dysfunction is very prevalent within the human population. The cellular 
defects that lead to SAN dysfunction are poorly understood and there is no permanent solution or cure for SAN dysfunction. In the medical setting, only the symptoms of SAN dysfunction are treated (Boink et al., 2015; Kurtz et al., 2010; Li et al., 2020). Through a stronger basic understanding of CPC cellular dynamics, principles relevant to pathobiological processes within the SAN can be uncovered and more efficient therapeutics to treat this disease may become possible.

Specifically, the concept of cellular-based or "biological" pacemakers has emerged as a potential approach for correcting chronic SAN dysfunction. For this to become viable, maintaining coupling boundaries between CPCs and the WM, establishing the proper ionic current densities, and sustaining size and structural characteristics among fabricated CPCs will be required (Boink et al., 2015; Li et al., 2020). Therefore, the major aim of this dissertation work is to show how such features are patterned developmentally with the goal that once we understand the critical cell biological events necessary for CPC formation, we can translate these to more effective treatments that will better suited for clinal settings. 


\section{REFERENCES}

Adler, C. P., \& Costabel, U. (1975). Cell number in human heart in atrophy, hypertrophy, and under the influence of cytostatics. Recent Adv Stud Cardiac Struct Metab, 6, 343-355. https://www.ncbi.nlm.nih.gov/pubmed/128080

Akiyama, T. (2010). Sunao Tawara: discoverer of the atrioventricular conduction system of the heart. Cardiol J, 17(4), 428-434. https://www.ncbi.nlm.nih.gov/pubmed/20690105

Alpert, N. R., Hamrell, B. B., \& Mulieri, L. A. (1979). Heart muscle mechanics. Annu Rev Physiol, 41, 521-537. https://doi.org/10.1146/annurev.ph.41.030179.002513

Anderson, R. H., \& Ho, S. Y. (1998). The architecture of the sinus node, the atrioventricular conduction axis, and the internodal atrial myocardium. J Cardiovasc Electrophysiol, 9(11), 12331248. https://doi.org/10.1111/j.1540-8167.1998.tb00097.x

Anderson, R. H., Janse, M. J., van Capelle, F. J., Billette, J., Becker, A. E., \& Durrer, D. (1974). A combined morphological and electrophysiological study of the atrioventricular node of the rabbit heart. Circ Res, 35(6), 909-922. https://doi.org/10.1161/01.res.35.6.909

Anderson, R. H., Spicer, D. E., Brown, N. A., \& Mohun, T. J. (2014). The development of septation in the four-chambered heart. Anat Rec (Hoboken), 297(8), 1414-1429. https://doi.org/10.1002/ar.22949

Anderson, R. H., Yanni, J., Boyett, M. R., Chandler, N. J., \& Dobrzynski, H. (2009). The anatomy of the cardiac conduction system. Clin Anat, 22(1), 99-113. https://doi.org/10.1002/ca.20700

Annunziato, L. (2013). Sodium calcium exchange: A growing spectrum of pathophysiological implications : proceedings of the 6th International Conference on Sodium Calcium Exchange. Springer. http://libproxy.lib.unc.edu/login?url=http://link.springer.com/10.1007/978-1-46144756-6

Aspelund, A., Robciuc, M. R., Karaman, S., Makinen, T., \& Alitalo, K. (2016). Lymphatic System in Cardiovascular Medicine. Circ Res, 118(3), 515-530. https://doi.org/10.1161/CIRCRESAHA.115.306544

Baluk, P., Fuxe, J., Hashizume, H., Romano, T., Lashnits, E., Butz, S., Vestweber, D., Corada, M., Molendini, C., Dejana, E., \& Mcdonald, D. M.. (2007). Functionally specialized junctions between endothelial cells of lymphatic vessels. Journal of Experimental Medicine, 204(10), 2349-2362. https://doi.org/10.1084/jem.20062596 
Bay, N. S., \& Bay, B. H. (2010). Greek anatomist herophilus: the father of anatomy. Anat Cell Biol, 43(4), 280-283. https://doi.org/10.5115/acb.2010.43.4.280

Bergwerff, M., Verberne, M. E., DeRuiter, M. C., Poelmann, R. E., \& Gittenberger-de Groot, A. C. (1998). Neural crest cell contribution to the developing circulatory system: implications for vascular morphology? Circ Res, 82(2), 221-231. https://doi.org/10.1161/01.res.82.2.221

Bestetti, R. B., Restini, C. B. A., \& Couto, L. B.. (2014). Development of Anatomophysiologic Knowledge regarding the Cardiovascular System: From Egyptians to Harvey. Arquivos Brasileiros De Cardiologia. https://doi.org/10.5935/abc.20140148

Bleeker, W. K., Mackaay, A. J., Masson-Pevet, M., Bouman, L. N., \& Becker, A. E. (1980). Functional and morphological organization of the rabbit sinus node. Circ Res, 46(1), 11-22. https://doi.org/10.1161/01.res.46.1.11

Bloom, W., Fawcett, DW. A textbook of histology. Chapman \& Hall; New York: 1994.

Boink, G. J., Christoffels, V. M., Robinson, R. B., \& Tan, H. L. (2015). The past, present, and future of pacemaker therapies. Trends Cardiovasc Med, 25(8), 661-673. https://doi.org/10.1016/j.tcm.2015.02.005

Bottani, S. (1995). Pulse-coupled relaxation oscillators: From biological synchronization to selforganized criticality. Phys Rev Lett, 74(21), 4189-4192. https://doi.org/10.1103/PhysRevLett.74.4189

Bouman, L. N., \& Jongsma, H. J. (1986). Structure and function of the sino-atrial node: a review. Eur Heart J, 7(2), 94-104. https://doi.org/10.1093/oxfordjournals.eurheartj.a062047

Boyett, M. R., Honjo, H., \& Kodama, I. (2000). The sinoatrial node, a heterogeneous pacemaker structure. Cardiovasc Res, 47(4), 658-687. https://doi.org/10.1016/s0008-6363(00)00135-8

Bressan, M., Henley, T., Louie, J. D., Liu, G., Christodoulou, D., Bai, X., Taylor, J., Seidman, C. E., Seidman, J. G., \& Mikawa, T. (2018). Dynamic Cellular Integration Drives Functional Assembly of the Heart's Pacemaker Complex. Cell Rep, 23(8), 2283-2291. https://doi.org/10.1016/j.celrep.2018.04.075

Brown, H. F., Kimura, J., Noble, D., Noble, S. J., \& Taupignon, A. (1984). The slow inward current, isi, in the rabbit sino-atrial node investigated by voltage clamp and computer simulation. Proc $\mathrm{R}$ Soc Lond B Biol Sci, 222(1228), 305-328. https://doi.org/10.1098/rspb.1984.0066 
Burnicka-Turek, O., Broman, M. T., Steimle, J. D., Boukens, B. J., Petrenko, N. B., Ikegami, K., Nadadur, R. D., Qiao, Y., Arnolds, D. E., Yang, X. H., Patel, V. V., Nobrega, M. A., Efimov, I. R., \& Moskowitz, I. P. (2020). Transcriptional Patterning of the Ventricular Cardiac Conduction System. Circ Res, 127(3), e94-e106. https://doi.org/10.1161/CIRCRESAHA.118.314460

Carmeliet, P., Ferreira, V., Breier, G., Pollefeyt, S., Kieckens, L., Gertsenstein, M., Fahrig, M., Vandenhoeck, A., Harpal, K., Eberhardt, C., Declercq, C., Pawling, J., Moons, L., Collen, D., Risau, W., \& Nagy, A. (1996). Abnormal blood vessel development and lethality in embryos lacking a single VEGF allele. Nature, 380(6573), 435-439. https://doi.org/10.1038/380435a0

Catanzariti, D., Maines, M., Manica, A., Angheben, C., Varbaro, A., \& Vergara, G. (2013). Permanent His-bundle pacing maintains long-term ventricular synchrony and left ventricular performance, unlike conventional right ventricular apical pacing. Europace, 15(4), 546-553. https://doi.org/10.1093/europace/eus313

Celesia, G. G. (2012). Alcmaeon of Croton's observations on health, brain, mind, and soul. J Hist Neurosci, 21(4), 409-426. https://doi.org/10.1080/0964704X.2011.626265

Chiesi, M., Ho, M. M., Inesi, G., Somlyo, A. V., \& Somlyo, A. P. (1981). Primary role of sarcoplasmic reticulum in phasic contractile activation of cardiac myocytes with shunted myolemma. J Cell Biol, 91(3 Pt 1), 728-742. https://doi.org/10.1083/jcb.91.3.728

dela Paz, N. G., \& D'Amore, P. A. (2009). Arterial versus venous endothelial cells. Cell Tissue Res, 335(1), 5-16. https://doi.org/10.1007/s00441-008-0706-5

Ferrara, N., Carver-Moore, K., Chen, H., Dowd, M., Lu, L., O'Shea, K. S., Powell-Braxton, L., Hillan, K. J., \& Moore, M. W. (1996). Heterozygous embryonic lethality induced by targeted inactivation of the VEGF gene. Nature, 380(6573), 439-442. https://doi.org/10.1038/380439a0

Galen. (2004). Galen's collected writings. Use of parts, vol. 12. Athens: Kaktos.

Gartner, LP.; Hiatt, JL. Color atlas of histology. Williams \& Wilkins; Baltimore: 1994.

Goethals, M., Raes, A., \& van Bogaert, P. P. (1993). Use-dependent block of the pacemaker current I(f) in rabbit sinoatrial node cells by zatebradine (UL-FS 49). On the mode of action of sinus node inhibitors. Circulation, 88(5 Pt 1), 2389-2401. https://doi.org/10.1161/01.cir.88.5.2389 
Goodyer, W. R., Beyersdorf, B. M., Paik, D. T., Tian, L., Li, G., Buikema, J. W., Chirikian, O., Choi, S., Venkatraman, S., Adams, E. L., Tessier-Lavigne, M., Wu, J. C., \& Wu, S. M. (2019). Transcriptomic Profiling of the Developing Cardiac Conduction System at Single-Cell Resolution. Circ Res, 125(4), 379-397. https://doi.org/10.1161/CIRCRESAHA.118.314578

Goodyer, W. R., \& Wu, S. M. (2018). Fates Aligned: Origins and Mechanisms of Ventricular Conduction System and Ventricular Wall Development. Pediatr Cardiol, 39(6), 1090-1098. https://doi.org/10.1007/s00246-018-1869-9

Haissaguerre, M., Vigmond, E., Stuyvers, B., Hocini, M., \& Bernus, O. (2016). Ventricular arrhythmias and the His-Purkinje system. Nat Rev Cardiol, 13(3), 155-166. https://doi.org/10.1038/nrcardio.2015.193

Hampton, H. R., Bailey, J., Tomura, M., Brink, R., \& Chtanova, T. (2015). Microbe-dependent lymphatic migration of neutrophils modulates lymphocyte proliferation in lymph nodes. Nat Commun, 6, 7139. https://doi.org/10.1038/ncomms8139

Herrmann, S., Lipp, P., Wiesen, K., Stieber, J., Nguyen, H., Kaiser, E., \& Ludwig, A. (2013). The cardiac sodium-calcium exchanger NCX1 is a key player in the initiation and maintenance of a stable heart rhythm. Cardiovascular research, 99(4), 780-788. https://doi.org/10.1093/cvr/cvt154

Ho, S. Y., Anderson, R. H., \& Sanchez-Quintana, D. (2002). Atrial structure and fibres: morphologic bases of atrial conduction. Cardiovasc Res, 54(2), 325-336. https://doi.org/10.1016/s0008-6363(02)00226-2

Ho, S. Y., \& Sanchez-Quintana, D. (2016). Anatomy and pathology of the sinus node. J Interv Card Electrophysiol, 46(1), 3-8. https://doi.org/10.1007/s10840-015-0049-6

Huang, W., Su, L., Wu, S., Xu, L., Xiao, F., Zhou, X., \& Ellenbogen, K. A. (2017). Benefits of Permanent His Bundle Pacing Combined With Atrioventricular Node Ablation in Atrial Fibrillation Patients With Heart Failure With Both Preserved and Reduced Left Ventricular Ejection Fraction. J Am Heart Assoc, 6(4). https://doi.org/10.1161/JAHA.116.005309

Huser, J., Blatter, L. A., \& Lipsius, S. L. (2000). Intracellular Ca2+ release contributes to automaticity in cat atrial pacemaker cells. J Physiol, $524 \mathrm{Pt}$ 2, 415-422. https://doi.org/10.1111/j.1469-7793.2000.00415.x

Hyer, J., Johansen, M., Prasad, A., Wessels, A., Kirby, M. L., Gourdie, R. G., \& Mikawa, T. (1999). Induction of Purkinje fiber differentiation by coronary arterialization. Proc Natl Acad Sci U S A, 96(23), 13214-13218. https://doi.org/10.1073/pnas.96.23.13214 
Issa, Z.F., Miller, J.M., Zipes, D.P.(2019). Molecular Mechanisms of Cardiac Electrical Activity. In Clinical Arrhythmology and Electrophysiology, 3rd ed.; Issa, Z.F., Miller, J.M., Zipes, D.P., Eds.; Elsevier: Philadelphia, PA, USA. pp. 1-14. https://doi.org/10.1016/B978-0-323-52356-1.00001-3

James, T. N. (2002). Structure and function of the sinus node, AV node and His bundle of the human heart: part I-structure. Prog Cardiovasc Dis, 45(3), 235-267. https://doi.org/10.1053/pcad.2002.130388

Joyner, R. W., \& van Capelle, F. J. (1986). Propagation through electrically coupled cells. How a small SA node drives a large atrium. Biophys J, 50(6), 1157-1164. https://doi.org/10.1016/S00063495(86)83559-7

Karkkainen, M.J., Haiko, P., Sainio, K., Partanen, J., Taipale, J., Petrova, T.V., Jeltsch, M., Jackson, D.G., Talikka, M., Rauvala, H., Betsholtz, C., Alitalo, K. (2004). Vascular endothelial growth factor $\mathrm{C}$ is required for sprouting of the firstnlymphatic vessels from embryonic veins. Nat Immunol. 2004;5:74-80. doi: 10.1038/ni1013.

Kaul, S. (1986). The interventricular septum in health and disease. Am Heart J, 112(3), 568-581. https://doi.org/10.1016/0002-8703(86)90523-5

Keith, A., \& Flack, M. (1907). The form and nature of the muscular connections between the primary divisions of the vertebrate heart. Journal of Anatomy and Physiology, 41, 172-189.

Kennedy, A., Finlay, D. D., Guldenring, D., Bond, R., Moran, K., \& McLaughlin, J. (2016). The Cardiac Conduction System: Generation and Conduction of the Cardiac Impulse. Crit Care Nurs Clin North Am, 28(3), 269-279. https://doi.org/10.1016/j.cnc.2016.04.001

Klasnja, A. V., Jakovljevic, D. G., Barak, O. F., Popadic Gacesa, J. Z., Lukac, D. D., \& Grujic, N. G.. (2013). Cardiac power output and its response to exercise in athletes and non-athletes. Clinical Physiology and Functional Imaging, 33(3), 201-205. https://doi.org/10.1111/cpf.12013

Kleber, A. G. (2011). Gap junctions and conduction of cardiac excitation. Heart Rhythm, 8(12), 1981-1984. https://doi.org/10.1016/j.hrthm.2011.08.007

Kohajda, Z., Loewe, A., Toth, N., Varro, A., \& Nagy, N. (2020). The Cardiac Pacemaker StoryFundamental Role of the $\mathrm{Na}(+) / \mathrm{Ca}(2+)$ Exchanger in Spontaneous Automaticity. Front Pharmacol, 11, 516. https://doi.org/10.3389/fphar.2020.00516 
Kozasa, Y., Nakashima, N., Ito, M., Ishikawa, T., Kimoto, H., Ushijima, K., Makita, N., \& Takano, M. (2018). HCN4 pacemaker channels attenuate the parasympathetic response and stabilize the spontaneous firing of the sinoatrial node. J Physiol, 596(5), 809-825.

https://doi.org/10.1113/JP275303

Kurtz, S. M., Ochoa, J. A., Lau, E., Shkolnikov, Y., Pavri, B. B., Frisch, D., \& Greenspon, A. J. (2010). Implantation trends and patient profiles for pacemakers and implantable cardioverter defibrillators in the United States: 1993-2006. Pacing Clin Electrophysiol, 33(6), 705-711. https://doi.org/10.1111/j.1540-8159.2009.02670.x

Lakatta, E. G., Maltsev, V. A., \& Vinogradova, T. M. (2010). A coupled SYSTEM of intracellular $\mathrm{Ca} 2+$ clocks and surface membrane voltage clocks controls the timekeeping mechanism of the heart's pacemaker. Circ Res, 106(4), 659-673.

https://doi.org/10.1161/CIRCRESAHA.109.206078

Li, Y., Wang, K., Li, Q., \& Zhang, H. (2020). Biological pacemaker: from biological experiments to computational simulation. J Zhejiang Univ Sci B, 21(7), 524-536.

https://doi.org/10.1631/jzus.B1900632

Liang, X., Wang, G., Lin, L., Lowe, J., Zhang, Q., Bu, L., Chen, Y., Chen, J., Sun, Y., \& Evans, S. M. (2013). HCN4 dynamically marks the first heart field and conduction system precursors. Circ Res, 113(4), 399-407. https://doi.org/10.1161/CIRCRESAHA.113.301588

Lu, J., Tong, X. Y., \& Wang, X. L. (2002). Altered gene expression of Na+/Ca2+ exchanger isoforms NCX1, NCX2 and NCX3 in chronic ischemic rat brain. Neurosci Lett, 332(1), 21-24. https://doi.org/10.1016/s0304-3940(02)00905-9

Lyashkov, A. E., Behar, J., Lakatta, E. G., Yaniv, Y., \& Maltsev, V. A. (2018). Positive Feedback Mechanisms among Local Ca Releases, NCX, and ICaL Ignite Pacemaker Action Potentials. Biophys J, 114(8), 2024. https://doi.org/10.1016/j.bpj.2018.03.024

Maass, K., Shekhar, A., Lu, J., Kang, G., See, F., Kim, E. E., Delgado, C., Shen, S., Cohen, L., \& Fishman, G. I. (2015). Isolation and characterization of embryonic stem cell-derived cardiac Purkinje cells. Stem Cells, 33(4), 1102-1112. https://doi.org/10.1002/stem.1921

Maltsev, V. A., \& Lakatta, E. G. (2008). Dynamic interactions of an intracellular Ca2+ clock and membrane ion channel clock underlie robust initiation and regulation of cardiac pacemaker function. Cardiovasc Res, 77(2), 274-284. https://doi.org/10.1093/cvr/cvm058 
Mangoni, M. E., \& Nargeot, J. (2008). Genesis and regulation of the heart automaticity. Physiol Rev, 88(3), 919-982. https://doi.org/10.1152/physrev.00018.2007

Markowitz, S. M., \& Lerman, B. B. (2018). A contemporary view of atrioventricular nodal physiology. J Interv Card Electrophysiol, 52(3), 271-279. https://doi.org/10.1007/s10840-0180392-5

Masson-Pevet, M., Bleeker, W. K., Mackaay, A. J., Bouman, L. N., \& Houtkooper, J. M. (1979). Sinus node and atrium cells from the rabbit heart: a quantitative electron microscopic description after electrophysiological localization. J Mol Cell Cardiol, 11(6), 555-568. https://doi.org/10.1016/0022-2828(79)90430-9

Masson-Pevet, M. A., Bleeker, W. K., Besselsen, E., Treytel, B. W., Jongsma, H. J., \& Bouman, L. N. (1984). Pacemaker cell types in the rabbit sinus node: a correlative ultrastructural and electrophysiological study. J Mol Cell Cardiol, 16(1), 53-63. https://doi.org/10.1016/s00222828(84)80714-2

Mavrodi, A., \& Paraskevas, G. (2014). Morphology of the heart associated with its function as conceived by ancient Greeks. Int J Cardiol, 172(1), 23-28. https://doi.org/10.1016/j.ijcard.2013.12.124

Midgett, M., Thornburg, K., \& Rugonyi, S. (2017). Blood flow patterns underlie developmental heart defects. Am J Physiol Heart Circ Physiol, 312(3), H632-H642. https://doi.org/10.1152/ajpheart.00641.2016

Miyamoto, T., Zhang, L., Sekiguchi, A., Hadama, T., \& Shimada, T. (2002). Structural differences in the cytoarchitecture and intercalated discs between the working myocardium and conduction system in the human heart. Heart Vessels, 16(6), 232-240. https://doi.org/10.1007/s003800200030

Mommersteeg, M. T., Hoogaars, W. M., Prall, O. W., de Gier-de Vries, C., Wiese, C., Clout, D. E., Papaioannou, V. E., Brown, N. A., Harvey, R. P., Moorman, A. F., \& Christoffels, V. M. (2007). Molecular pathway for the localized formation of the sinoatrial node. Circ Res, 100(3), 354-362. https://doi.org/10.1161/01.RES.0000258019.74591.b3

Monfredi, O., Maltseva, L. A., Spurgeon, H. A., Boyett, M. R., Lakatta, E. G., \& Maltsev, V. A. (2013). Beat-to-Beat Variation in Periodicity of Local Calcium Releases Contributes to Intrinsic Variations of Spontaneous Cycle Length in Isolated Single Sinoatrial Node Cells. PLoS One, 8(6), e67247. https://doi.org/10.1371/journal.pone.0067247 
Munshi, N. V. (2012). Gene regulatory networks in cardiac conduction system development. Circ Res, 110(11), 1525-1537. https://doi.org/10.1161/CIRCRESAHA.111.260026

Nicenboim, J., Malkinson, G., Lupo, T., Asaf, L., Sela, Y., Mayseless, O., Gibbs-Bar, L., Senderovich, N., Hashimshony, T., Shin, M., Jerafi-Vider, A., Avraham-Davidi, I., Krupalnik, V., Hofi, R., Almog, G., Astin, J. W., Golani, O., Ben-Dor, S., Crosier, P. S., Herzog, W., Lawson, N. D., Hanna, J. H., Yanai, I., \& Yaniv, K. (2015). Lymphatic vessels arise from specialized angioblasts within a venous niche. Nature, 522(7554), 56-61. https://doi.org/10.1038/nature14425

Olszewski, W. L. (2003). The lymphatic system in body homeostasis: physiological conditions. Lymphat Res Biol, 1(1), 11-21; discussion 21-14. https://doi.org/10.1089/15396850360495655

Olszewski, W. L., Grzelak, I., Ziolkowska, A., \& Engeset, A. (1995). Immune cell traffic from blood through the normal human skin to lymphatics. Clin Dermatol, 13(5), 473-483. https://doi.org/10.1016/0738-081x(95)00087-v

Opthof, T., de Jonge, B., Jongsma, H. J., \& Bouman, L. N. (1987). Functional morphology of the pig sinoatrial node. J Mol Cell Cardiol, 19(12), 1221-1236. https://doi.org/10.1016/s00222828(87)80532-1

Opthof, T., de Jonge, B., Masson-Pevet, M., Jongsma, H. J., \& Bouman, L. N. (1986). Functional and morphological organization of the cat sinoatrial node. J Mol Cell Cardiol, 18(10), 1015-1031. https://doi.org/10.1016/s0022-2828(86)80290-5

Park, D.S., Fishman, G.I. (2011). The cardiac conduction system. Circulation. 123(8): 904-15.

Petrova, T. V., \& Koh, G. Y. (2018). Organ-specific lymphatic vasculature: From development to pathophysiology. J Exp Med, 215(1), 35-49. https://doi.org/10.1084/jem.20171868

Reuter, H., \& Seitz, N. (1968). The dependence of calcium efflux from cardiac muscle on temperature and external ion composition. J Physiol, 195(2), 451-470. https://doi.org/10.1113/jphysiol.1968.sp008467

Sabin, F.R. (1902). On the origin of the lymphatic system from the veins and the development of the lymph hearts and thoracic duct in the pig. Am J Anat, 1:367-389.

Sanchez-Quintana, D., Cabrera, J. A., Farre, J., Climent, V., Anderson, R. H., \& Ho, S. Y. (2005). Sinus node revisited in the era of electroanatomical mapping and catheter ablation. Heart, 91(2), 189-194. https://doi.org/10.1136/hrt.2003.031542 
Sedmera, D., \& Gourdie, R. G. (2014). Why do we have Purkinje fibers deep in our heart?. Physiological research, 63(Suppl 1), S9-S18. https://doi.org/10.33549/physiolres.932686

Sharma, P. S., Vijayaraman, P., \& Ellenbogen, K. A. (2019). Author Correction: Permanent His bundle pacing: shaping the future of physiological ventricular pacing. Nat Rev Cardiol, 16(12), 760. https://doi.org/10.1038/s41569-019-0240-Z

Sherwood, L. Human physiology: from cells to systems. Thomson Brooks/Cole; Belmont: 2007.

Silverman ME, Grove D, Upshaw CH. (2006). Why does the heart beat? The discovery of the electrical system of the heart. Circulation. 113: 2775-2781.

Sternbach, G.L., Varon, J., Fromm, R.E., Sicuro, M., Baskett, P.J. (2009). Galen and the origins of artificial ventilation, the arteries and the pulse. Resuscitation. 49(2):119-22.

Tan, L.B. (1987). Clinical and research implications of new concepts in the assessment of cardiac pumping performance in heart failure. Cardiovasc Res. 21: 615-622.

Tawara S. 2000. The conduction system of the mammalian heart. In: An Anatomico-histological Study of the Atrioventricular Bundle and the Purkinje Fibers. [Trans. Suma K, Shimada M.] London: Imperial College Press.

Trautwein, W., \& Uchizono, K.. (1963). Electron microscopic and electrophysiologic study of the pacemaker in the sino-atrial node of the rabbit heart. Cell and Tissue Research, 61(1), 96-109. https://doi.org/10.1007/bf00341523

Unudurthi, S. D., Wolf, R. M., \& Hund, T. J. (2014). Role of sinoatrial node architecture in maintaining a balanced source-sink relationship and synchronous cardiac pacemaking. Front Physiol, 5, 446. https://doi.org/10.3389/fphys.2014.00446

van Eif, V. W. W., Devalla, H. D., Boink, G. J. J., \& Christoffels, V. M. (2018). Transcriptional regulation of the cardiac conduction system. Nat Rev Cardiol, 15(10), 617-630. https://doi.org/10.1038/s41569-018-0031-y

Van Praagh, R., \& Van Praagh, S. (1983). Aristotle's "triventricular" heart and the relevant early history of the cardiovascular system. Chest, 84(4), 462-468.

https://doi.org/10.1378/chest.84.4.462 
van Weerd, J. H., \& Christoffels, V. M. (2016). The formation and function of the cardiac conduction system. Development, 143(2), 197-210. https://doi.org/10.1242/dev.124883

Vedantham, V. (2015). New Approaches to Biological Pacemakers: Links to Sinoatrial Node Development. Trends Mol Med, 21(12), 749-761. https://doi.org/10.1016/j.molmed.2015.10.002

Vedantham, V., Galang, G., Evangelista, M., Deo, R. C., \& Srivastava, D. (2015). RNA sequencing of mouse sinoatrial node reveals an upstream regulatory role for Islet-1 in cardiac pacemaker cells. Circ Res, 116(5), 797-803. https://doi.org/10.1161/CIRCRESAHA.116.305913

Verhoeff, K., \& Mitchell, J. R. (2017). Cardiopulmonary physiology: why the heart and lungs are inextricably linked. Adv Physiol Educ, 41(3), 348-353. https://doi.org/10.1152/advan.00190.2016

Vinogradova, T. M., Zhou, Y. Y., Bogdanov, K. Y., Yang, D., Kuschel, M., Cheng, H., \& Xiao, R. P. (2000). Sinoatrial node pacemaker activity requires $\mathrm{Ca}(2+) /$ calmodulin-dependent protein kinase II activation. Circ Res, 87(9), 760-767. https://doi.org/10.1161/01.res.87.9.760

Vinogradova, T. M., Zhou, Y. Y., Maltsev, V., Lyashkov, A., Stern, M., \& Lakatta, E. G. (2004). Rhythmic ryanodine receptor $\mathrm{Ca} 2+$ releases during diastolic depolarization of sinoatrial pacemaker cells do not require membrane depolarization. Circ Res, 94(6), 802-809. https://doi.org/10.1161/01.RES.0000122045.55331.0F

von der Weid, P. Y., \& Zawieja, D. C. (2004). Lymphatic smooth muscle: the motor unit of lymph drainage. Int J Biochem Cell Biol, 36(7), 1147-1153. https://doi.org/10.1016/j.biocel.2003.12.008

Wiig, H., \& Swartz, M. A. (2012). Interstitial fluid and lymph formation and transport: physiological regulation and roles in inflammation and cancer. Physiol Rev, 92(3), 1005-1060. https://doi.org/10.1152/physrev.00037.2011

Winfree, A. T. (1967). Biological rhythms and the behavior of populations of coupled oscillators. J Theor Biol, 16(1), 15-42. https://doi.org/10.1016/0022-5193(67)90051-3

Woods, W. T., Imamura, K., \& James, T. N. (1982). Electrophysiological and electron microscopic correlations concerning the effects of neuraminidase on canine heart cells. Circ Res, 50(2), 228239. https://doi.org/10.1161/01.res.50.2.228

Woods, W. T., Urthaler, F., \& James, T. N. (1976). Spontaneous action potentials of cells in the canine sinus node. Circ Res, 39(1), 76-82. https://doi.org/10.1161/01.res.39.1.76 
Wu, J., Schuessler, R. B., Rodefeld, M. D., Saffitz, J. E., \& Boineau, J. P. (2001). Morphological and membrane characteristics of spider and spindle cells isolated from rabbit sinus node. Am $\mathrm{J}$ Physiol Heart Circ Physiol, 280(3), H1232-1240.

https://doi.org/10.1152/ajpheart.2001.280.3.H1232

Zawieja, D. C. (2009). Contractile physiology of lymphatics. Lymphat Res Biol, 7(2), 87-96. https://doi.org/10.1089/1rb.2009.0007 


\section{CHAPTER 2: OPTICAL ELECTROPHYSIOLOGY IN THE DEVELOPING HEART ${ }^{1}$}

\section{Introduction}

The heart is an electrochemically oscillating muscular syncytium that generates the biomechanical forces required to pump fluid through the circulatory system. As for all muscle beds in the body, cardiac activation is stimulated by voltage-dependent changes in plasma membrane ion permeability. Large changes in the ion current flow trigger the self-sustaining electrical impulses, or action potentials, required for contraction. Electrical propagation in the heart is highly regulated by a specialized subpopulation of cells referred to as the cardiac conduction system (CCS). The CCS is completely contained within the heart and can rhythmically initiate and coordinate the impulse propagation required for cardiac function independent of any external stimuli.

In the mature heart, the CCS is comprised of the sinoatrial node (SAN), the atrioventricular node (AVN), the bundle of His, bundle branches and Purkinje fibers (Figure 4A). The electrical impulses that induce contraction are initiated by pacemaker cells within the SAN and are then rapidly conducted across the atrial myocardium. Propagation is then slowed as it reaches the AVN, allowing time for the atria to contract and fluid to move prior to activation of the ventricular chambers of the heart. The impulse then travels through the His bundle, bundle branches, and the Purkinje system, enabling coordinated contraction of the large ventricular chambers of the heart

\footnotetext{
${ }^{1}$ This chapter previously appeared as an article in the Journal of Cardiovascular Development and Disease. The original citation is as follows: Thomas K, Goudy J, Henley T, Bressan M. Optical electrophysiology in the developing heart. Journal of cardiovascular development and disease. 05/2018;5(2):28. doi:10.3390/jcdd5020028.
} 
A
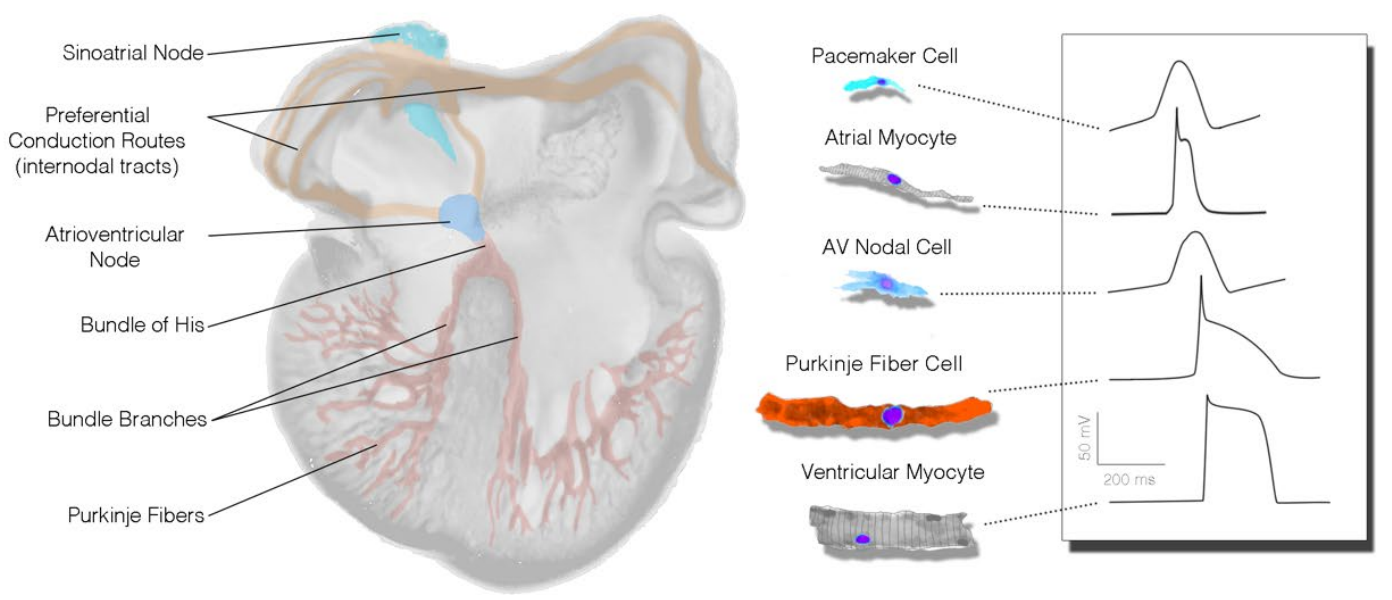

Figure 4: Organization of the mature cardiac conduction system.

A) Schematic of a four-chambered heart indicating the relative positions of the specialized subcomponents of the cardiac conduction system; (B) Relative cytoarchitecture and action potential characteristics of cells from various conduction system and working myocardial populations.

Importantly, each subcomponent of the CCS is largely unique in both form and function from other regions of the heart. Anatomically, cells of the SAN and AVN are generally smaller than other myocardial populations, lack highly organized sarcomeres, and have relatively simple membrane structures (no intercalated disks or T-tubule systems) (Opthof et al., 1985; Opthof et al., 1987; Anderson et al., 1998; James et al., 1966; James et al., 1968). These cells are also coupled through relatively high resistance gap junctions (Joyner et al., 1986; Boyett et al., 2000; Gourdie et al., 1993) and rely heavily on calcium currents for the upstroke of their action potentials (Schram et al., 2002; Hagiwara et al., 1988; Mesirca et al., 2015). As a result of these anatomic and molecular characteristics, electrical propagation through SAN and AVN cells tends to be very slow.

Features present in nodal cells are quite different from the rapidly conducting components of the His-Purkinje system. While species differences exist, in general, Purkinje fiber cells are larger than the working ventricular myocardium (Ono et al., 2009), express elevated levels of the low-resistance gap junctional protein Cx40 (Gourdie et al., 1993 and Kanter et al., 1993), and utilize rapidly activating 
sodium currents for depolarization (Vaidyanathan et al., 2013 and Colatsky et al., 1979). Consistent with nodel cells, however, His-Purkinje cells also tend to lack dense sarcomeres and display few T-tubules (Shimada et al., 2004).

From a physiological standpoint, the most prominent feature of the nodal populations is the presence of slow diastolic depolarization (Figure 4B). During the heart's resting phase, nodal cell membranes slowly depolarize as a collection of mutually entrained cell-surface ion channels, pumps, and sarcoplasmic reticulum-based proteins allow for cytoplasmic accumulation of positively charged ions. This serves to depolarize the cell membrane, stimulating voltage-dependent ion channels to open, which allows the initiation of an action potential (Schram et al., 2002; Verkerk et al., 2007; Maltsev et al., 2007; Lakatta et al., 2010). Cells within the SAN have the fastest intrinsic rate of activity and therefore serve as the primary pacemaking region of the heart. His-Purkinje cells can be identified physiologically on the basis of parameters including a rapid upstroke velocity, a more negative resting membrane potential than the working myocardium, and an elongated action potential duration (Figure 4B) (Schram et al., 2002 and Balati et al., 1998).

Interestingly, as the heart forms and becomes functional in the embryo, none of the cell types present within the mature CCS are present. Determining how the complex cellular circuitry that comprises the CCS is organized and integrated into the existing developmental framework of the heart has, therefore, remained one of the most fascinating and challenging topics associated with embryological cardiac morphogenesis. It has now become apparent that components of the conduction system become functional in a proximal-to-distal sequence, with the pacemaker cells of the SAN differentiating during heart looping stages (Bressa et al., 2014), preferential conduction tracts through the atria forming during early septation stages (Bressan et al., 2014 and Viragh et al., 1982), the AVN and His bundle emerging towards the end of ventricular septation (Sedmera et al., 2006 and Viragh et al., 1982), and the ventricular Purkinje fiber network maturing following the completion of cardiac morphogenesis (Hyer et al., 1999; Gourdie et al., 1998; Takeybayashi-Suzuki et al., 2000; Zhang et al., 2011). As such, the CCS represents a 
biorhythmic cellular network that forms and becomes functional within a field of pre-existing electrically active working myocardial cells, meaning that each cell population within the conduction system must acquire specialized electrophysiological characteristics and establish the appropriate connections with the adjacent components of the network while the heart is actively beating. Given the complexity of cardiac morphogenesis, examining how these processes occur has, and continues, to require unique physiological tools. A major focus of this review is to introduce some of the imaging techniques that have helped to demonstrate the functional maturation of the developing heart, provide a brief historical perspective on the adaption of those techniques for embryonic cardiac physiology, and project how novel imaging and cell biological techniques may help to push the field forward in the coming years.

\section{Historical Perspective}

While our modern understanding of the cellular, molecular, and physiological events that dictate electrical patterning of the heart continues to reveal unexpected details, the underlying fascination with the rhythmic, coordinated beating of the developing heart extends back millennia. Much of this interest has stemmed from the simple satisfaction provided by watching the rhythmic cycle of contraction in the embryonic heart. Indeed, observational accounts of the developing avian heartbeat date as far back as Aristotle (Needham, 1939). It is not surprising, therefore, that much of the research focused on trying to understand the nature and developmental emergence of cardiac biorythmicity has initially been based on visual observation. For instance, as early as 1890, Fano and Bandano constructed an ingenious method of projecting light though embryonic cardiac tissue onto moving film. In doing so, their studies produced the first recordings of embryonic heart activity. In this pioneering study, regional differences in the innate rate of activation between subdomains of the heart were first documented, the requirement of the atrioventricular junction for synchronization of the forming atria and ventricles was noted, and the speed of signal transmission in the early heart was first estimated at $6-8 \mathrm{~cm} / \mathrm{s}$ (reviewed by (Patten, 1949; Kamino, 1991; De Mello, 1982). The advent of a microscopy technique referred to as micromoving pictures by Patten (Patten, 1933) further pushed the boundaries of early developmental cardiac research. 
In 1920, Sabin published the first report capturing the timing and location of the initial contractions of the embryonic chick heart tube, and, expanding on this, Patten and Kramer were able to construct a detailed analysis of how the first contractions of the heart related to the morphogenetic fusion of the embryonic cardiac primordia (Patten, 1933). Predictably, an array of film-based recording studies followed these early reports, as researchers began to recognize that visual recordings simultaneously provided large amounts of both spatial and temporal information regarding the activity of the heart, a concept that remains a principle component of the optical techniques used today for developmental cardiac physiology.

In parallel with these early visual-recording-based studies, the electrical nature of the developmental cardiac activity was first explored as electrocardiograph techniques were adopted for use on embryonic tissue. In 1913, Wertheim-Salomonson succeeded in detecting extracellular electrical fluctuations from chick embryos. While hampered by the sensitivity of the detection equipment used, these studies were able to demonstrate clear electrical deflections initiated in developing hearts (Patten, 1933). With improvements in technology, in particular, the development of signal amplifiers, a cohort of investigators were able to record electrical activity from successively younger embryos spanning looping stages of cardiac morphogenesis through septation stages (Hoff et al., 1939; Bogue, 1933; Eyster et al., 1937). Perhaps most significantly, these investigators were able to demonstrate that features present in the adult echocardiogram (ECG) could be detected in hearts as young as late looping stages, confirming the multi-chambered activation sequence present in the adult heart (atria followed by ventricles) emerging during early embryonic development.

The ability to directly record changes in membrane potential via intracellular microelectrodes was first utilized in the developing heart in a series of studies conducted by Fingl, Woodbury, and Hecht. In these studies, the maturation of the electrical impulse shape over cardiac development was first noted, and how the developmental cardiac action potential responded to chemical treatment was explored (Fingl et al., 1952). In the 1960s, Van Mierop was able to expand on this work, becoming the first investigator to 
record action potentials from cardiomyocytes as early as heart tube stages, and noting that regional differences in action potential kinetics emerge during looping morphogenesis (Van Mierop et al., 1967). The subsequent progress of electrical recordings of developing cardiomyocytes has been extensively reviewed elsewhere (Sperelakis et al., 1983); however, the impact that these earliest studies have had on our fundamental understanding of the electrical maturation of the heart cannot be overstated.

Throughout the first half of the 20th century, both visual recording and electrical sampling were used independently to trace how morphogenesis and physiology cooperatively evolve as the heart forms. However, it did not escape investigators' attention that technical limitations with both techniques challenged the ability to develop a comprehensive model of cardiac functional maturation. While video recordings provided an overview of organ-level activation and could be used to construct longitudinal studies of cardiac development, data was largely based on the physical movement of the tissue. As this is an indirect measure of physiological behavior, the underlying regional differences present among various populations of cardiomyocytes could not be directly assessed. In contrast, electrical recordings provided detailed information regarding the kinetics of membrane dynamics in various regions of the heart, but these studies were technically challenging and provided only limited spatial information, making quantification of parameters including the directionality of impulse propagation and the conduction velocity relatively inaccessible. In addition, the inability to impale small cells ( $<3-5 \mathrm{~m}$ in diameter) with microelectrodes and the fact that contractile movement frequently disrupts the membrane seals required for stable recording represent significant challenges for conducting such developmental cardiac electrophysiology studies. Therefore, it is not surprising that a merger of optical recording and electrical sampling emerged as a method to circumvent the limitations of these techniques, as being able to directly visualize electrical activity simultaneously from many sites in a tissue preparation without physically disrupting the sample combined the most attractive components of each technique. As summarized in Figure 5, the ability to quantitatively examine electrical activity across a whole tissue can be used to 
examine a variety of physiological parameters in ways that would be exceedingly challenging using other methodologies.
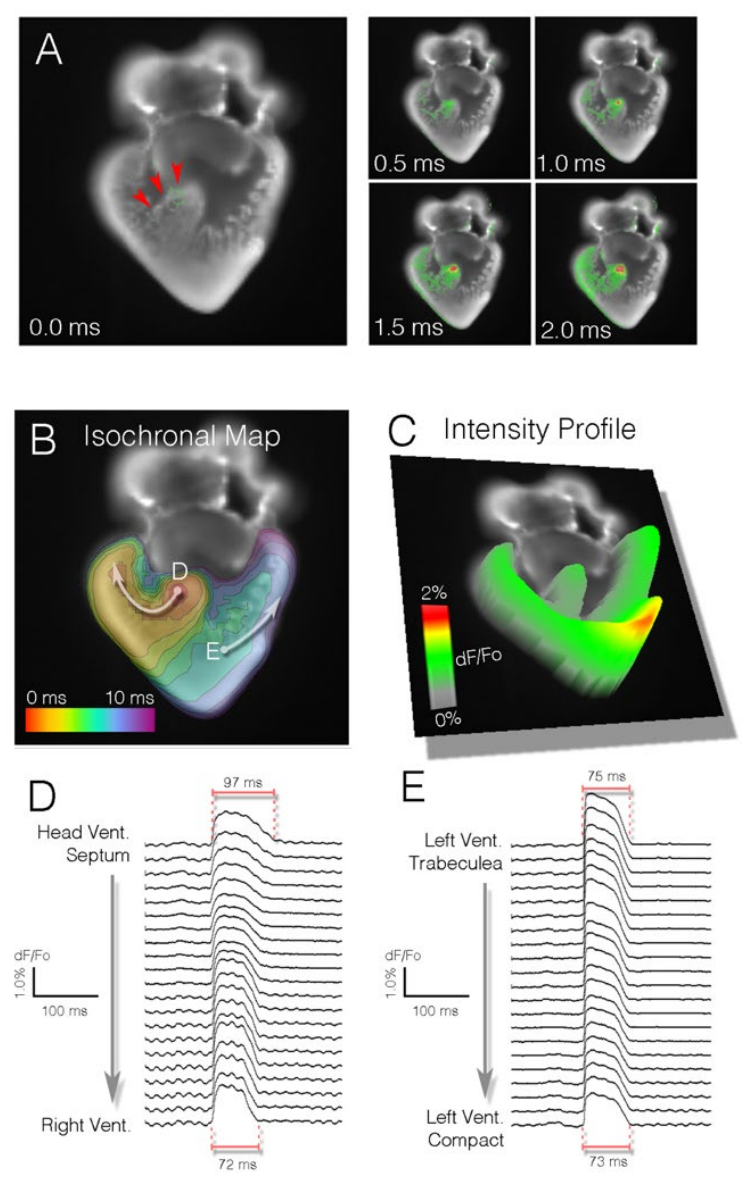

Figure 5. Optical mapping allows for integrative, simultaneous acquisition of physiological parameters from multiple points in situ.

(A) Time series of electrical impulse propagation through a section of embryonic cardiac tissue. In this example, a Hamburger and Hamilton (HH) stage 30 (Hamburger et al., 1951) embryonic chick heart was sectioned in the coronal plane and live imaged at 2000 frames per second. Activation of the right bundle branch (red arrowheads) can be seen; (B) Isochronal map (1 ms/div) depicting the ventricular activation pattern of the heart from (A); (C) Intensity plot indicating relative signal amplitude $(\mathrm{dF} / \mathrm{Fo})$ for heart in $(\mathrm{A})$; (D) Stripe analysis from region noted in (B). These data track the change in action potential shape as the impulse moves from the His bundle towards the right ventricle. Note that action potential duration decreases; (E) As in (D), tracing of action potential characteristics as it propagates from left ventricular trabeculae to compact myocardium.

\section{Optical Mapping for Developmental Cardiac Studies}

The push to develop optical techniques for monitoring electrical activity was most prominently

driven by researchers in neuroscience. The vast majority of what is known regarding the voltage-

dependent membrane currents that drive changes in membrane permeability comes from either 
intracellular or patch clamp measurements of membrane potentials. While extraordinarily powerful, these techniques require a large degree of expertise and have a few critical limitations. From the perspective of a neuroscientist, microelectrode-based techniques are not able to monitor behavior at multiple sites along a cell; nor are they fine cytoarchitectural features accessible for recording (i.e., small dendritic or axonal projections). As such, starting in the 1960s, optical techniques for examining changes in membrane potential began to be explored, with the underlying rationale being that visual recording of electrical activation could be used to directly evaluate changes in membrane potential from multiple regions of cells/tissue without the risk of physical damage. The list of techniques, reagents, and indicators that have been developed for such purposes is quite expansive (Canepari et al., 2015), and, given the focus of this review, we have chosen to highlight the evolution of reagents that have been widely used in the developing heart.

\section{$\underline{\text { Voltage-Sensitive Dyes }}$}

Initial endeavors at tracking electrical activity with optical instrumentation were based on birefringence principles (Hill et al., 1948 and Cohen et al., 1974). The theory behind this was that changes in electrical field intensity across a membrane influence the polarization or light-scattering properties of that membrane, which could be projected to a detector such as a photomultiplier. While such techniques are still utilized [45], they suffer from poor signal amplitude and a low signal-to-noise ratio. As a result, attempts were made to screen fluorescent dyes for spectral shifts that correlated with changes in membrane potential (Cohen et al., 1974; Ross et al., 1977, Gupta et al., 1981). Most membrane depolarization events in adult excitable cells have a magnitude of approximately $100 \mathrm{mV}$. While this is relatively small when compared to most of the electronic systems we routinely interact with, when considerations for membrane geometry are made, millivolt differences across a cell membrane can support voltage gradients greater than $105 \mathrm{~V} / \mathrm{cm} 2$ (Bucher et al., 1969). Operating under the hypothesis that electrical fields of this size should be sufficient to alter the optical absorption/emission characteristics of chromophores, thousands of dyes were initially evaluated for potentiometric activity. This led to the 
initial identification of merocynanine dyes as having characteristics sufficient for voltage imaging (Cohen et al., 1974; Ross et al., 1977, Gupta et al., 1981). These dyes responded to voltage changes with a linear shift in fluorescence intensity, displayed rapid spectral shifts as cells depolarized, and had adequate signal-to-noise ratios to allow for direct monitoring without substantial signal averaging.

Following the initial identification of the merocynanine dyes, Leslie Loew's laboratory conducted a series of rational design studies in an effort to further improve and optimize dyes for live imaging of voltage changes in excitable tissues (Loew et al., 1979 and Loew et al., 1979). These studies yielded many of the voltage-sensitive dyes (VSDs) that have been and are still widely used for optical imaging of voltage changes (Fluhler et al., 2002). Among these, Di-4-anepps has been, perhaps, the most popular potentiometric dye used for embryonic heart studies.

Di-4-anepps was engineered with several features that make it well suited for developmental cardiac imaging. Di-4-anepps belongs to a class of Styryl dyes that show rapid response and a relatively large change in fluorescence per millivolt (mV)of depolarization (Habib-E-Rasul et al., 2013). The amplitude of the Di-4-anepps response is particularly useful in preparations of embryonic cardiac tissue, for which cells achieve only a fraction of the membrane polarization seen in adult cardiomyocyte (Sperelakis et al.,1972). In general, the structure of Di-4-anepps can be subdivided into three functional regions: a hydrophobic group for membrane anchoring, a voltage-sensitive chromophore, and a charged moiety to orient the dye molecule perpendicular to the cell membrane and slow internalization. The chromophore can be further subdivided into an electron-rich pi system, linker, and electron-deficient pi system. Collectively, these features allow the dye to embed into the outer leaflet of the plasma membrane (via a pair of hydrophilic hydrocarbon chains) in an orientation parallel to the electrical field generated by the cell's membrane potential. Excitation of the dye shifts the electron configuration in a manner that is sensitive to the local electrical field, resulting in membrane-potential-dependent alterations in emission wavelength (Loew et al., 1978) (Figure 6A). As a cell depolarizes, the dye shifts its emission spectra, which can be detected as a decrease in intensity in longer (red) wavelengths in favor of increased intensity 
in shorter (blue) wavelengths. This change in fluorescence $(\mathrm{dF} / \mathrm{Fo})$ is linearly associated with changes in membrane potential and can be used to monitor and trace waves of depolarization (Figure 6B).

Instrumentation is, therefore, designed to capture these rapid changes in fluorescence intensity. While Di4-anepps is widely used, some of its limitations should be noted. Di-4-anepps has been shown to alter action potential kinetics (particularly conduction velocity and action potential duration) with prolonged imaging (Schaffer et al., 1994 and Larsen et al., 2012), and, as with all potentiometric dyes, Di-4-anepps does not provide an absolute value for membrane potential, only a relative change in fluorescence.

\section{Calcium Dyes}

As with VSDs, calcium indicators have been broadly applied to both neural and cardiac imaging modalities to trace cellular excitability. Membrane depolarization in cardiomyocytes leads to the opening of cell-surface voltage-dependent, calcium-selective ion channels. This results in calcium ion influx, which triggers a release of calcium from intracellular sarcoplasmic reticulum stores. Calcium-induced calcium release drives cytosolic calcium to increase from nanomolar up to millimolar concentrations, triggering sarcomere contraction. Therefore, monitoring changes in the cytosolic calcium concentration is an effective, albeit indirect, method of visualizing cardiac electrical propagation. Several classes of calcium dyes have been generated over the years, with those most prevalently used including the Indo, Quin, Fura, and Fluo dyes. Each have undergone multiple rounds of iteration to optimize fluorescence, permeability, toxicity, and kinetics, and dozens of indicators are now commercially available (Paredes et al., 2008). These indicators all share an octacoordinate binding site based on the structure of the calcium selective chelator EGTA (Tsien, 1980 and Tsien, 1989). When occupied by a calcium ion, this region of the dye molecule elicits structural changes in the attached fluorophore, leading to calcium-dependent increases in fluorescence (Figure 6C). While these indicators have slower on/off kinetics than potentiometric dyes such as Di-4-anepps, the change in fluorescence upon electrical stimulation are orders of magnitude higher than VSDs. This can be of great utility for measuring activity from immature cardiomyocytes, for which shallow polarization (and thus low action potential magnitude) can limit the 
effectiveness of VSDs. Just as with VSDs, considerations should be made for the structure and nature of how these indicators work when selecting them for use. One primary concern when using calcium indicators is choosing a compound with the appropriate affinity. High-affinity dyes can act as intracellular calcium buffers, actively interfering with normal cycling dynamics and distorting measurements.

Therefore, the most accurate measurement of calcium dynamics is in fact achieved by using an indicator with the lowest calcium affinity that can be practically imaged. This often requires testing a few reagents across the spectrum of potential dyes. For instance, we have tested several calcium dyes with developing avian cardiac tissues, including Fura-2 $(\mathrm{Kd} \sim 140 \mathrm{~nm})$, Rhod-2 $(\mathrm{Kd} \sim 570 \mathrm{nM})$, and Flou5F $(\mathrm{Kd} \sim 2.3$ $\mathrm{uM})$, and found that Cal520 ( $\mathrm{Kd} \sim 235 \mathrm{nM})$ provides effective signal-to-noise and strong intensity shifts when imaged from heart tube to looping stages (Figure 6D).
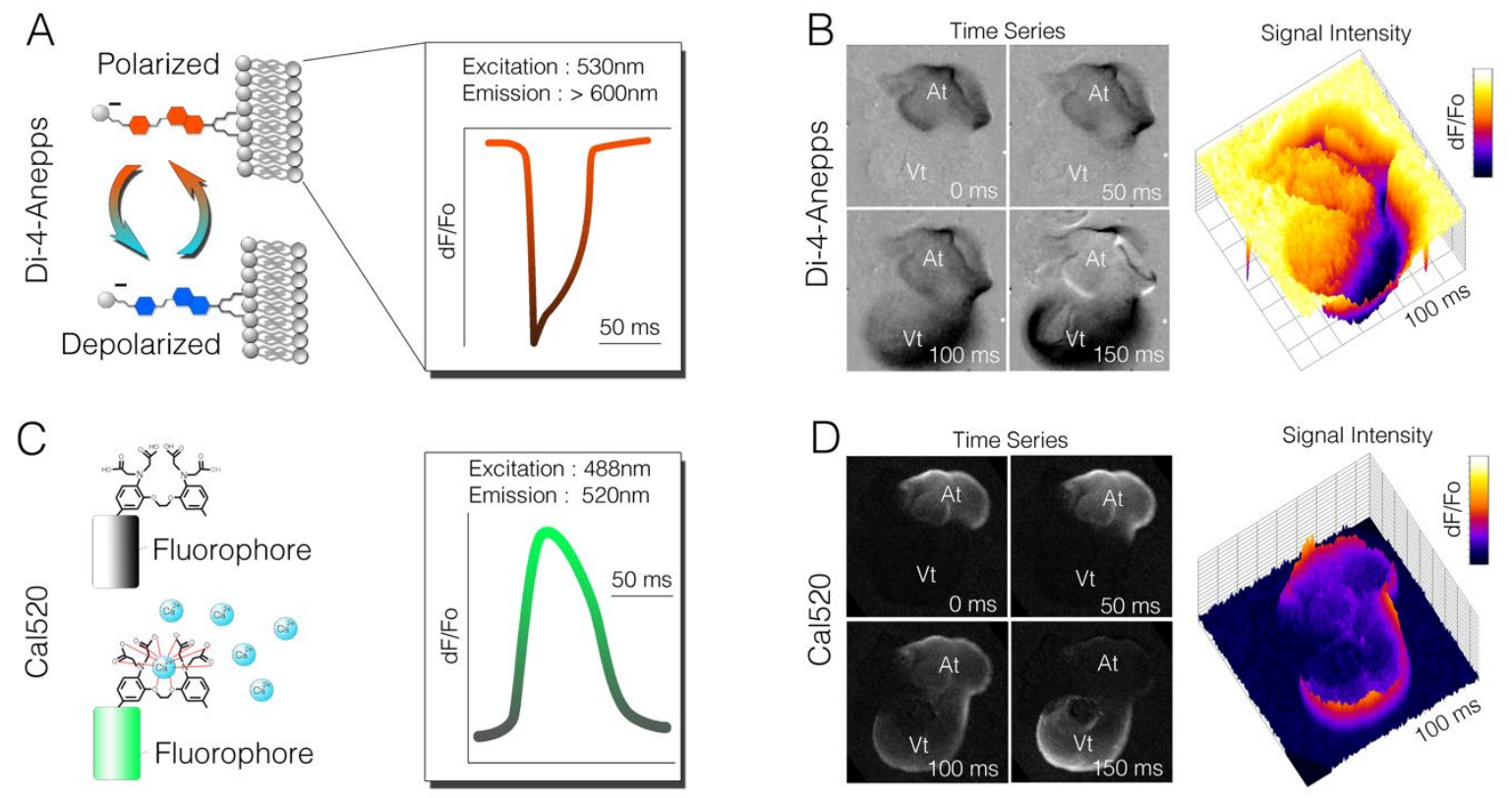

Figure 6. Comparison of voltage-sensitive dyes to small-molecule calcium indicators.

(A) Diagram indicating change in spectral characteristics for the voltage-sensitive dye, Di-4-anepps. Depolarization causes a blue shift in the dye. In this example, an imaging system was designed to image long wavelengths (red), and a drop in fluorescence intensity correlated to an action potential being fired; (B) Time series of raw imaging data acquired from a Hamburger and Hamilton $(\mathrm{HH})$ stage 18 chick heart stained with D-4-annepps. The drop in intensity was seen as the cardiac action potential propagated from atria to ventricle; (C) Diagram of the smallmolecule calcium indicator Cal520. Calcium ion occupancy in the ETGA/BAPTA-like motif of the molecule increased fluorescence output, which could be imaged with most standard eGFP filter sets; (D) Raw imaging of $\mathrm{Ca} 50 \mathrm{dF} / \mathrm{Fo}$ in a HH stage heart. Unlike Di-4-anepps (B), Cla520 increased its fluorescence signal upon depolarization. 


\section{Genetically Encoded Indicators}

While both potentiometric and ion-sensitive dyes have been used with great success for tracking physiological dynamics in the developing heart, improvements in genetically encoded indicators (GEIs) for voltage and calcium have greatly broadened the available tool kit for cardiac imaging. As with the dye-based approaches discussed above, GEIs have gone through multiple rounds of design-based iteration in order to achieve sensitivity, dynamic ranges, and signal-to-noise ratios comparable to small-molecule indicators. A large array of GEIs now exist, with differing spectral characteristics, affinities, and intensities (Palmer et al.., 2006 and Rodriquez et al., 2017). Among the most appealing aspects of the GEIs is that they do not require a staining procedure, which can be difficult to target to specific cell types. Cell-type-specific promoters can be utilized to drive the expression of GEIs in specific domains in the embryo, and, unlike the dye-based strategies, physiological behaviors can be monitored over prolonged periods. Furthermore, GEIs are being increasingly used to perform high-throughput evaluation of functional characteristics in stem-cell or somatic-cell reprogramming techniques for generating cardiomyocytes (Rodriquez et al., 2017; Addis et al., 2013; Huebsch et al., 2015; Shiba et al., 2012; Chong et al., 2014; Schinnawi et al., 2015), in which staining procedures and dye toxicity are major limitations. Thus far, the most widely used GEIs for calcium imaging in the developing heart are based on GCaMP. GCaMP was engineered to contain a circularly permuted eGFP flanked by the M13 fragment of myosin light-chain kinase on one side and calmodulin on the other. In the presence of calcium ions, a calcium-camodulin-M13 interaction is stabilized, resulting in a conformational change in the eGFP that increases its fluorescence output (Nakai et al., 2001) . GCaMP2 was the first genetically encoded calcium indicator to be used to evaluate calcium transient behavior in a developing four-chambered heart (Tallini et al., 2006), and successive improvements on the basic structure of GCaMP have led to its use in a variety of developmental model organisms.

While the panel of genetically encoded voltage sensors for optical recording is expanding at a rapid rate (Xu et al., 2017), their use in developmental cardiac studies remains far less prevalent than 
calcium indicators. As these reagents improve, however, it is likely that their inclusion in developmental studies will become more common.

\section{Basic Instrumentation}

Instrumentation for physiological imaging of the developmental heart does require some specialization to accommodate challenges that are not necessarily as significant in adult tissue. As mentioned above, developing cardiomyocytes are not as polarized as their adult counterparts (Sperelakis et al., 1972), particularly before ventricular septation has completed. This effectively means the amplitude of the action potentials that juvenile cardiomyocytes exhibit can be quite small and, as a result, changes in fluorescent indicator intensities are not as pronounced as in adult tissue. Furthermore, the developing heart is, of course, far smaller than in the adult. The fewer cells and thinner wall thickness present during development mean that the volume of excitable cells from which a signal can be recorded is far less than in the adult. As such, imaging systems for developmental studies must be optimized for maximal lightcollecting ability.

Currently there are a multitude of imaging components that can be combined to construct a basic microscopy system for optical mapping purposes. In general, the major considerations in designing these systems are related to speed, magnification, and sensitivity of light detection.

A stable, high-intensity light source is preferable for any optical mapping system. The mercury arc light sources used for most fluorescent imaging techniques are robust and bright and have a large array of flexible uses, but tend to show too much fluctuation in output intensity over the millisecond time scales in which physiological imaging studies are conducted. Tungsten halogen lamps display improved stability over mercury arc systems; however, halogen bulbs that can generate sufficient intensity have relatively short operational lives (approx. $100 \mathrm{~h}$ ). As such, laser systems and light-emitting diodes (LEDs) are now the most common forms of illumination.

Most developmental imaging systems require at least some marginal magnification capacity, although this can vary greatly depending on the model organism (e.g., a fully looped zebrafish heart is 
approximately $200 \mathrm{~m}$ in length, whereas a fully septated chick heart is approximately $1 \mathrm{~cm}$ in length). In general, the objective characteristics most ideal for developmental optical mapping are a long working distance and high numerical aperture. Typically, trade-offs must be made between these two parameters to meet the needs of the sample to be examined.

As with objectives, similar trade-offs must also be made when selecting detectors for optical mapping. Generally, this trade-off manifests in sacrificing speed and sensitivity for the number of pixels that can be simultaneously imaged or vice versa. For example, the upstroke component of a cardiac action potential is in the order of $2 \mathrm{~ms}$ (Hirota et al., 1979). In order to acquire multiple time points during the upstroke event, imaging may need to be conducted at $1-2 \mathrm{kHz}$. Very few camera systems with megapixel densities are capable of imaging at that speed while also being able to detect small deviations in fluorescence intensity. Historically, photodiode arrays were the first detectors used for optical mapping in cardiac tissues. These were fairly low resolution systems consisting of only a few sampling regions (Kamino, 1991). However, the photodiode arrays provided a high dynamic range, high acquisitions speeds, and good signal-to-noise. Progressively, advancements in CCD and CMOS camera chip technologies have supplanted the earlier photodiode arrays. The major advantages with CCD and CMOS cameras were the increased pixel density they could achieve and the relative ease of use. The last decade has seen an uptick in cameras manufactured expressly for optical mapping techniques, with notable companies including RedShirt Imaging (Decatur, GA, USA) and SciMedia (Costa Mesa, CA, USA), who each offer a variety of camera systems with differing temporal and spatial specifications. However, when imaging for calcium transient behavior (typically on the order of 50-100 frames per second), more standard, commercially available cameras, including those from Hamamatsu (Bridgewater, NJ, USA) and Andor (Belfast, UK), which have a higher spatial resolution, are often adequate.

In summary, reagents and equipment for conducting a variety of imaging-based investigations into the electrical maturation of the heart are now widely available. The diversity and flexibility of these tools is opening opportunities to investigate developmental cardiac physiology from excitingly novel 
perspectives. As described below, effective application of these techniques has already provided fundamental insight into the structure-function dynamics of cardiac morphogenesis.

\section{Application of Physiological Imaging to Understand Cardiac Electrophysiological Maturation}

Through a series of imaging studies spanning the last 40 years, a wealth of detail has emerged regarding how developmental transitions in cardiac morphogenesis correlate to changes in electrophysiological properties. Pioneering work in this field was initiated by Kohtaro Kamino's group, who began using potentiometric dyes to evaluate electrical activity in cardiac progenitors as well as in the early heart tube (Kamino, 1991; Hirota et al., 1979; Hirota et al., 1983; Fujii et al., 1981). Given the limitations of intracellular recording techniques noted above, these studies were among the first to examine electrical activation and propagation at these early stages of cardiac development. Strikingly, through a series of reports, the imaging system designed by the Kamino lab was able to identify electrical activity in the early lateral plate mesoderm, prior to the initial fusion of the progenitor cells that make up the heart tube, well before the onset of cardiac contraction (Patten, 1949; Hirota et al., 1979; Hirota et al., 1983). Initially, electrical activity was restricted to a small region of the mesoderm, and the amplitude of the recorded signals was quite small (Patten, 1949). However, over successive stages, the region of the heart primordia that appeared to be spontaneously excitable expanded, resulting in propagating waves that moved through the pre-contractile myocardium. Recent studies using the calcium indicator Cal520 have confirmed that propagating transients are present during cardiac crescent stages in the mouse embryo, consistent with the studies conducted in the chick almost 40 years ago (Tyser et al. 2016).

As the heart tube initially forms, the electrically active area is larger than the contractile mass (Bressan et al., 2013; Van Mierop, 1967; Arguello et al., 1986). At these stages, impulses initiate in the left posterior inflow segment of the heart tube and propagate as a uniform wave toward the most anterior segment of the heart (Bressan et al., 2013; Van Mierop, 1967; Fujii et al., 1981), whereas only a small region of the more anterior myocardium displays contractile movement (Patten, 1949) (Figure 7A). Parameters of the electrical impulse are quite immature at these stages, displaying relatively slow 
upstroke velocities and elongated action potential durations (Van Mierop, 1967; Arguello et al., 1986; Bressan et al., 2014). Furthermore, the frequency of activation is also slow, as the cycle length between successive electrical impulses can exceed 1-2 s.

Regional differences in conduction characteristics emerge as the heart undergoes cardiac looping. The conduction velocity begins to increase along the outer curvature of the forming ventricle as the heart begins to loop (Breesan et al., 2013; Sedmera et al., 2004; Ma et al., 2014) (Figure 7B), and, coincidently with the emergence of the endocardial cardiac cushions, a region of slow conduction emerges at the atrioventricular junction separating the embryonic atrial and ventricular chambers (Van Mierop, 1967 and Bressan et al., 2014). This marks the embryonic equivalent of the slow conducting AVN present in the mature heart. The activation pattern of the heart at this stage of development remains unidirectional, initiating in the inflow region of the heart, propagating across the primitive atria, and then proceeding from the base of the ventricle towards the cardiac outflow.

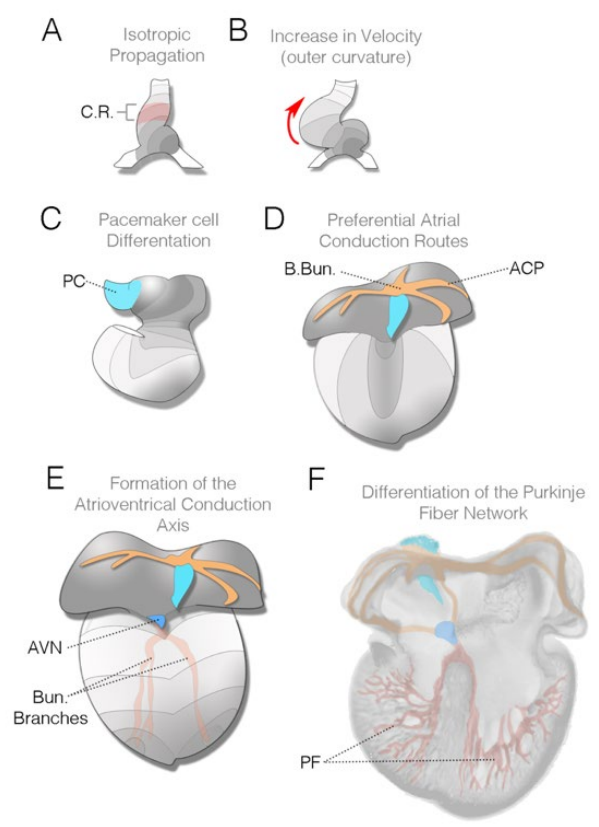

Figure 7. Changes in conduction patterning during cardiac morphogenesis.

(A) Diagram of heart tube viewed from the ventral surface (Hamburger and Hamilton (HH) stage 10 in chick, E8.25 in mouse). This stage corresponds to the first observable contractions in the heart; contractile region (C.R.) is noted in red. Electrical impulses initiate in the left inflow region of the heart (grey region) and propagate as a uniform wave towards the outflow; (B) As cardiac looping progresses (HH stage 15 in chick, E8.5 in mouse), the impulse 
increases velocity along the outer curvature of the heart (red arrow); (C) Towards the end of looping, cardiac pacemaker cells (PC) differentiate and are added to the heart (light-blue region). These cells will make up the muscular component of the mature sinoatrial node (HH stage 18 in chick, E9.5 in mouse); (D) Diagram of an early septation stage heart viewed from the dorsal surface (HH stage 23-25 in chick, E10.5-E12.5 in mouse). During these stages, atrial muscle bundles, including Bachmann's bundle, elaborate and establish themselves as atrial conduction pathways (ACP_orange); (E) As ventricular septation completes, the atrioventricular conduction complex, including the atrioventricular node (AVN; dark blue), His bundle, and bundle branches (red) become functional HH stage 25-30 in chick. As noted in the text and in Figure 5, the right bundle branch frequently becomes active prior to the left bundle branch; (F) Dorsal half of the heart viewed from the front. After the termination of cardiac morphogenesis, the distal Purkinje fiber (PF) network differentiates in subepicardial and/or perivascular regions in the heart. All diagrams are based on chick cardiac development.

Towards the end of cardiac looping, pacemaker cells emerge within the right inflow component of the heart (Bressan et al., 2013). Unlike the atrial and ventricular primordium that are added to the heart at earlier developmental stages and appear to transition through intermediate electrically active states, bona fide pacemaker cells show functional characteristics far closer to their adult counterparts when they first differentiate. Optical recordings demonstrate that these cells display a pronounced slow diastolic depolarization and a much higher frequency of depolarization than either atrial or ventricular progenitors (Bressan et al., 2013). Around the same time that pacemaker cells are differentiating, subtle changes to ventricular activation patterns are also occurring. Imaging from chick, mouse, and rat embryos demonstrates that the conduction pattern present in the ventricles begins to evolve as a propagation route emerges along the ventral anterior surface of the myocardium, roughly corresponding to where the ventricular septum will form (Sedmera et al., 2004; Sedmera et al., 2005; Rentschler et al., 2001). Interestingly, this pattern emerges prior to the completion of ventricular septation and precedes the formation of the His bundle and bundle branches.

As looping terminates, the atrial conduction pattern begins to transition. Initially, conduction across the atria proceeds as an even isotropic wave moving from the pacemaking region to the atrioventricular junction; however, as the pectinate muscle bundles of the atria form and enlarge, these structures become preferential conduction conduits through the atrial myocardium (Sedmera, 2006 and Bressan et al., 2014) (Figure 7D). A large bundle along the roof of the atria, known as Bachmann's bundle, becomes one of the principle routes by which impulses generated in the SAN move from the right atria to the left (Sedmera, 2006 ). More generally, however, as pacemaker-initiated action potentials enter 
the roof of the atria, the large pectinate muscles coordinate atrial activation by rapidly conducting impulses towards the forming atrioventricular conduction axis. While the atrial muscle bundles do not meet the formal anatomical definition of a specialized conduction network (Anderson et al., 1988), recent reports have indicated that they are enriched with several factors associated with a rapid conduction velocity, including CX40 and SCN5a (Bressan et al., 2014 and Benes, et al., 2014).

Slow conduction through the entire atrioventricular myocardium persists through the completion of cardiac looping and is eventually terminated by the formation of an insulating fibrous ring between the atria and ventricles. This prevents electrical communication between the upper and lower chambers of the heart, except through the forming atrioventricular conduction axis. As the ventricular septum partitions the right and left ventricles, conduction through the bundle branches can be detected (Figures 5 and 7E). Optical mapping studies performed on a variety of vertebrate models have demonstrated that the right bundle branch appears to become functional prior to the left bundle branch (Sedmera et al., 2005 and Rentschler et al., 2001) (Figure 5). This formally marks the shift in the ventricular activation pattern from what is known as base-to-apex, to apex-to-base. The functional activation of the bundle branches precedes the establishment of the definitive ventricular fast-conducting conduction network consisting of Purkinje fibers. The Purkinje fiber network is the last component of the heart's conduction system to terminally differentiate, becoming active during late gestational periods in avians and after birth in mice (Hyer et al., 1999 and Zhang et al., 2011).

In general, studies focused on directly observing transitions in the heart's conduction patterns as morphogenesis progresses have established that drastic changes in activations patterns occur during cardiac development. Currently, the vast majority of these studies have been limited to imaging embryos using two-dimensional modalities. Moving forward, adaptive technologies that can integrate multidimensional imaging over several developmental windows will be required to push our understanding of how the electrical syncytium of the heart patterns. Several recent studies have fairly nicely demonstrated how the basic principles of physiological imaging outlined above are currently being 
supplemented by modern optical technologies to create new insights into cardiac electrophysiological maturation.

\section{Recent Advancements}

The ability to visualize impulse propagation across whole tissues has been critical for determining the stages at which cardiac conduction characteristics change and mature towards adult physiology. However, because of limitations born from the requirement of maximal light collection and high temporal resolution, much of the data collected to date regarding developmental cardiac physiology has not been multidimensional. This is by no means a critique of previous data or methodology, but the limitations of extrapolating what are essentially two-dimensional imaging series onto the true three-dimensional (3D) structure of the heart can lead to errors in calculating functional characteristics. For instance, it is difficult to account for electrical propagation pathways moving toward or away from a detector when considering absolute conduction velocity, and out of focus cells can complicate the interpretation of even ideal conduction pathways that are traversing perpendicular to the detector.

With this in mind, several studies have begun to push the imaging systems for optical physiology in the heart towards multidimensional interfaces. In a few such studies, a custom-built optical mapping system with adequate magnification specifications to image develop quail cardiac tissue was integrated onto an optical coherence tomography (OCT) platform. OCT is a non-invasive imaging modality based on measuring backscattered light intensity using low-coherence interferometry (Yelbuz, 2002). It does not require the introduction of contrast reagents and is non-destructive. Given its relatively high spatial resolution $(2-20 \mathrm{~m})$, good penetration depths $(1-3 \mathrm{~mm})$, and high sampling rates $(47 \mathrm{kHz})$, it can be used to rapidly construct 3D profiles of tissue architecture. Combining OCT with optical mapping allowed for data collected by imaging VSD responses to be mapped onto a 3D surface rendering of a mid-loopingstage quail heart. This allowed for the correction of conduction velocity speeds across the $3 \mathrm{D}$ architecture of the heart (Ma et al., 2014). Subsequent studies using a combined OCT/OM imaging system have 
demonstrated that computational processing based on least squares optimization can further refine the 3D extrapolation of the conduction velocity in developing cardiac tissue (Gu et al., 2015).

Efforts have also been made to directly acquire 3D physiological data from embryonic cardiac tissue. Using a custom-built light-sheet microscope, Ma et al. were able to scan through a developing quail heart and computationally reconstruct an averaged electrical impulse propagation pathway to generate complete four-dimensional, volumetric recordings of cardiac activation maps (Ma et al., 2016).

Similarly, using the zebrafish heart, Weber et al. developed a light-sheet imaging system capable of creating high-speed imaging of calcium transient behavior across development (Weber, 2015). This report demonstrated a scalable imaging system that can be used to quantify organ-level physiological dynamics but that also possesses sufficient resolution to quantify individual cellular behavior at multiple stages of cardiac morphogenesis.

The ability to stimulate and simultaneously record cardiac activity using a non-invasive, nongenetic, optical approach was recently explored by Wang et al. (Wang et al., 2014). Using pulses of infrared point stimulation, the authors were able to effectively capture the heart rate without having to apply and an electrode-based stimulator.

The convergence of advanced fluorescent probes, genetic indicators, and imaging platforms is increasingly removing barriers towards both optically based recordings and light-induced manipulation of developing cardiac tissue. As such, it is not difficult to project the biological applications that studies in the near future will push these technologies towards.

\section{Future Directions}

Moving forward, a major challenge for developmental cardiac physiological imaging will be to develop methods that will allow for single-cell or subcellular resolution of functional dynamics in the forming heart. As current stem-cell and tissue engineering strategies for generating cardiomyocytes continue to advance, detailed understanding of the in vivo cellular and physiological maturation of cardiomyocytes will become increasingly relevant for the development of novel cellular-based 
therapeutics. A major technical barrier towards pushing stem-cell-based or regenerative medicine application towards clinical use is the relatively juvenile nature of cardiomyocytes that are generated through these techniques (Wang et al., 2014; Veerman et al.,2015; Scuderi et al., 2017; Alcon et al., 2012). In this respect, direct physiological examination and manipulation at single-cell or subcellular resolution will provide critical insight for optimizing approaches for generating such cells ex vivo.

In addition, congenital heart defects (CHDs) represent the leading class of birth defects seen in humans. Correspondingly, considerable effort has been dedicated to understanding the molecular genetics of CHDs; however, how mutations in various pathways manifest at the level of the individual cellular function remains poorly understood. The major platforms available for studying genotype/phenotype relationships in developing cardiomyocytes have been dependent on the generation of transgenic model organisms or, more recently, the differentiation of patient-derived induced pluripotent stem cells (Tzatzalos et al., 2016; Smith et al., 2017). While transgenic animal models represent the most powerful systems currently available for CHD research, it can be difficult to tease apart cell autonomous effects that result from genetic manipulation from those that are secondary to organ-level dysfunction. As such, iPSC systems represent a strong alternative approach; however, iPSC-derived cardiomyocytes are difficult to mature and lack the biomechanical environmental cues and electrical circuitry present in the heart (Bedada et al., 2016).

An alternative system to complement current transgenic animal-based manipulations may, therefore, be one that utilizes genetic and somatic transgenic approaches to generate mosaic cardiac tissues. The rationale behind such studies would be to engineer systems in which only small numbers of cells within the developing heart are manipulated, leaving the bulk of cardiac tissue in a wild-type configuration. Such approaches have several potential advantages. From the standpoint of physiological imaging, genetically encoded biosensors can be expressed in subpopulations of cells. The cells that carry the optically detectable biosensor can be easily identified while remaining directly in contact with their neighbors, and the behavior of the individual or small groups of cells can be assayed while maintaining 
the electromechanical syncytium of the heart. This allows for optimal imaging in the developing heart as single cells can be resolved against a background of negative cells (Figure 8). Unlike full transgenic animals, for which the vast majority of cells are labeled, such a mosaic system would allow for fine subcellular events to be live imaged in vivo. While not yet broadly utilized in developmental cardiac research, inspiration for this type of approach can again be drawn from neuroscience research.

Particularly in regard to physiological imaging, several strategies for creating mosaic neural networks, in which subpopulations of cells are labeled and examined, have been used with great success.

A classical approach toward understanding single-cell functional dynamics within the brain involves delivering small-molecule dyes specifically to cells of interest via a patch-clamp pipette. While effective for local delivery, this approach can be technically challenging, and the calcium/voltage reporters used for such studies are typically not amenable to long-term imaging, as their phototoxicity precludes longitudinal studies. Furthermore, it can be challenging to target such dyes to specific cell types during microinjection. The use of GEIs, however, has effectively been applied to circumvent these issues. Approaches including viral gene delivery and in vivo electroporation have been utilized to directly introduce molecular tools for optical physiology in the brains of wild-type or genetically modified animals (Knopfel, 2012 and Tian, 2014). Both of these systems can take advantage of localized targeting; cell-type-specific promoter designs; Cre/FLP recombinases; and, in the case of viruses, differential infectivity to generate the level and specificity of integration required for the desired experimental conditions.

Viral-based somatic transgenesis has been used in cardiac research for over 20 years (Mikawa et al., 1996; Ong et al., 1998; Mikawa et al., 2006; Cohen-Gould et al., 1996). Retroviral-based genetic lineage tracing and overexpression studies are commonly performed in avian embryos, and several recent studies in rodents have demonstrated the utility of adeno-associated virus (AAV) for the in vivo generation of mosaic cardiac tissue (Carroll et al., 2016 and Guo et al., 2017). Therefore, viral-mediated somatic transgenesis is an attractive approach for generating myocardial populations for in situ single-cell 
live imaging. In addition, while electroporation is difficult to perform in the developing heart, recent studies have demonstrated that various chemical-based transfection techniques are possible during early avian cardiac morphogenesis (Ishii et al., 2010 and Jordan et al., 2014). Indeed, our group has conducted initial proof-of-concept studies in the chick embryo, confirming that transfection-based introduction of the calcium indicator GCaMP6F can be used to generate cardiac mosaics in which calcium transient behavior can be imaged across different stages of cardiac development at the subcellular resolution (Figure 8). Moving forward, it is not difficult to foresee experimental approaches in which diseaserelevant pathways are disrupted in cells co-transfected with physiological indicators, allowing for the functional consequences of genetic perturbations to be read out in real-time. Furthermore, given the success of optogenetic approaches in the adult heart and developing heart (Arrenberg et al., 2010; Bruegmann et al., 2010; Nussinovitch et al., 2015; Burton et al., 2015), somatic transgenic approaches open an exciting avenue towards conducting light-based manipulations of both physiological and biochemical processes in developing cardiomyocytes in vivo with a high degree of targeting specificity. In summary, the imaging and molecular biology approaches that are coming online across the spectrum of in vivo and in vitro biomedical research are drastically altering the way cellular physiology is interrogated. As developmental cardiac research continues to elaborate and construct tractable means of porting these techniques into the embryonic heart, the technical hurdles towards the systematic, scalable investigation of cardiac physiological maturation are becoming less prohibitive, opening exciting and novel means to investigate the mechanisms of cardiac functional maturation. 

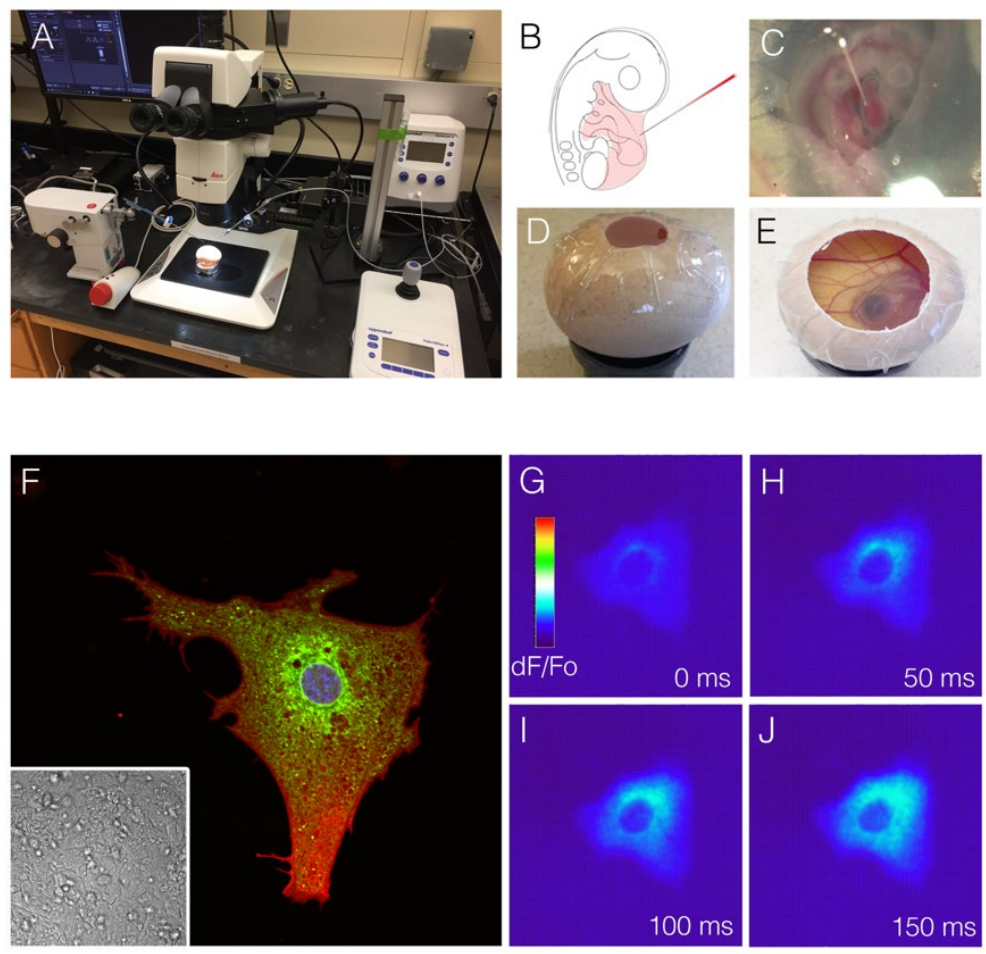

Figure 8. Mosaic analysis for imaging cardiac cell physiology maturation.

(A) Microinjection system for viral- or plasmid-based transduction of cardiac cells; (B) Diagram of microinjection into the pericardial space of a chick embryo; (C) Photograph of injection procedure from (B); (D,E) Sealing and incubation of microinjected embryo to desired stage; (F) Embryonic cardiomyocyte transfected in vivo with a construct containing a membrane-targeted RFP, a sarcoplasmic-reticulum-targeted eGFP, and a nuclear-targeted BFP. This cell exists within a field of untransfected cardiomyocytes, as indicated by the phase contrast image (inset); (G-J) Time series of a cardiomyocyte from a similar stage as that depicted in (F), transfected with a construct encoding for the calcium indicator GCaMP6F. Cell was imaged at 100 frames per second. Calcium transients can be seen initiating in a perinuclear region (similar to the dense area of sarcoplasmic reticulum from (F)) and propagating out towards the cell periphery. 


\section{REFERENCES}

Addis, R.C.; Ifkovits, J.L.; Pinto, F.; Kellam, L.D.; Esteso, P.; Rentschler, S.; Christoforou, N.; Epstein, J.A.; Gearhart, J.D. Optimization of direct fibroblast reprogramming to cardiomyocytes using calcium activity as a functional measure of success. J. Mol. Cell. Cardiol. 2013, 60, 97-106.

Alcon, A.; Cagavi Bozkulak, E.; Qyang, Y. Regenerating functional heart tissue for myocardial repair. Cell. Mol. Life Sci. 2012, 69, 2635-2656.

Anderson, R.H.; Ho, S.Y. The Architecture of the Sinus Node, the Atrioventricular Conduction Axis, and the Internodal Atrial Myocardium. J. Cardiovasc. Electrophysiol. 1998, 9, 1233-1248.

Arrenberg, A.B.; Stainier, D.Y.R.; Baier, H.; Huisken, J. Optogenetic Control of Cardiac Function. Science 2010, 330, 971-974.

Argüello, C.; Alanís, J.; Pantoja, O.; Valenzuela, B. Electrophysiological and ultrastructural study of the atrioventricular canal during the development of the chick embryo. J. Mol. Cell. Cardiol. 1986, $18,499-510$.

Balati, B.; Varro, A.; Papp, J.G. Comparison of the cellular electrophysiological characteristics of canine left ventricular epicardium, M cells, endocardium and Purkinje fibres. Acta Physiol. Scand. 1998, 164, 181-190.

Badreddine, A.H.; Jordan, T.; Bigio, I.J. Real-time imaging of action potentials in nerves using changes in birefringence. Biomed. Opt. Express 2016, 7, 1966-1973.

Bedada, F.B.; Wheelwright, M.; Metzger, J.M. Maturation status of sarcomere structure and function in human iPSC-derived cardiac myocytes. BBA Mol. Cell Res. 2016, 1863, 1829-1838.

Benes, J., Jr.; Ammirabile, G.; Sankova, B.; Campione, M.; Krejci, E.; Kvasilova, A.; Sedmera, D. The role of connexin40 in developing atrial conduction. FEBS Lett. 2014, 588, 1465-1469.

Bogue, J.Y. The Electrocardiogram of the Developing Chick. J. Exp. Biol. 1933, 10, 286-292.

Boyett,M.R.; Honjo, H.; Kodama, I. The sinoatrial node, a heterogeneous pacemaker structure. Cardiovasc. Res. 2000, 47, 658-687. 
Bressan, M.; Liu, G.; Mikawa, T. Early mesodermal cues assign avian cardiac pacemaker fate potential in a tertiary heart field. Science 2013, 340, 744-748.

Bressan, M.; Yang, P.B.; Louie, J.D.; Navetta, A.M.; Garriock, R.J.; Mikawa, T. Reciprocal myocardialendocardial interactions pattern the delay in atrioventricular junction conduction. Development 2014, 141, 4149-4157.

Bressan, M.C.; Louie, J.D.; Mikawa, T. Hemodynamic For ces Regulate Developmental Patterning of Atrial Conduction. PLoS ONE 2014, 9, e115207.

Bruegmann, T.; Malan, D.; Hesse, M.; Beiert, T.; Fuegemann, C.J.; Fleischmann, B.K.; Sasse, P. Optogenetic control of heart muscle in vitro and in vivo. Nat. Meth. 2010, 7, 897-900.

Bücher, H.; Wiegand, J.; Snavely, B.B.; Beck, K.H.; Kuhn, H. Electric field induced changes in the optical absorption of a merocyanine dye. Chem. Phys. Lett. 1969, 3, 508-511.

Burton, R.A.B. Optical control of excitation waves in cardiac tissue. Nat. Photonics 2015, 9, 813-816.

Canepari, M.; Zecevic, D.; Bernus, O. Membrane Potential Imaging in the Nervous System and Heart; Springer:Cham, Switzerland, 2015.

Carroll, K.J.;Makarewich, C.A.;McAnally, J.; Anderson, D.M.; Zentilin, L.; Liu, N.; Giacca,M.; BasselDuby, R.; Olson, E.N. A mouse model for adult cardiac-specific gene deletion with CRISPR/Cas9. Proc. Natl. Acad. Sci. USA 2016, 113, 338-343.

Chong, J.J.H.; Yang, X.; Don, C.W.; Minami, E.; Liu, Y.-W.; Weyers, J.J.; Mahoney, W.M.; Van Biber, B.; Cook, S.M.; Palpant, N.J.; et al. Human embryonic-stem-cell-derived cardiomyocytes regenerate non-human primate hearts. Nature 2014, 510, 273-277. J. Cardiovasc. Dev. Dis. 2018, 5,2818 of 19

Cohen, L.B.; Hille, B.; Keynes, R.D. Light scattering and birefringence changes during activity in the electric organ of Electrophorus electricus. J. Physiol. 1969, 203, 489-509. J. Cardiovasc. Dev. Dis. 2018, 5, 2817 of 19

Cohen-Gould, L.; Mikawa, T. The fate diversity of mesodermal cells within the heart field during chicken early embryogenesis. Dev. Biol. 1996, 177, 265-273. 
Colatsky, T.J.; Tsien, R.W. Sodium channels in rabbit cardiac Purkinje fibres. Nature 1979, 278, $265-$ 268.

Davis, L.M.; Kanter, H.L.; Beyer, E.C.; Saffitz, J.E. Distinct gap junction protein phenotypes in cardiac tissues with disparate conduction properties. J. Am. Coll. Cardiol. 1994, 24, 1124-1132.

De Mello,W.C. Intercellular communication in cardiac muscle. Circ. Res. 1982, 51.

Eyster, J.A.E.; Krasno, M.R.; Hettwer, J.P. Electrical potentials of the heart of the chick embryo. Am. J. Physiol. 1937, 120, 173-178.

Gourdie, R.G.; Severs, N.J.; Green, C.R.; Rothery, S.; Germroth, P.; Thompson, R.P. The spatial distribution and relative abundance of gap-junctional connexin 40 and connexin 43 correlate to functional properties of components of the cardiac atrioventricular conduction system. J. Cell Sci. 1993, 105, 985-991.

Gourdie, R.G.;Wei, Y.; Kim, D.; Klatt, S.C.; Mikawa, T. Endothelin-induced conversion of embryonic heart muscle cells into impulse-conducting Purkinje fibers. Proc. Natl. Acad. Sci. USA 1998, 95, $6815-6818$.

Gu, S.; Wang, Y.T.; Ma, P.; Werdich, A.A.; Rollins, A.M.; Jenkins, M.W. Mapping conduction velocity of early embryonic hearts with a robust fitting algorithm. Biomed. Opt. Express 2015, 6, $2138-$ 2157.

Guo, Y.; VanDusen, N.J.; Zhang, L.; Gu,W.; Sethi, I.; Guatimosim, S.;Ma, Q.; Jardin, B.D.; Ai, Y.; Zhang, D.; et al. Analysis of Cardiac Myocyte Maturation Using CASAAV, a Platform for Rapid Dissection of Cardiac Myocyte Gene Function In Vivo. Circ. Res. 2017, 120, 1874-1888.

Gupta, R.K.; Salzberg, B.M.; Grinvald, A.; Cohen, L.B.; Kamino, K.; Lesher, S.; Boyle, M.B.;Waggoner, A.S.; Wang, C.H. Improvements in optical methods for measuring rapid changes in membrane potential. J. Membrain Biol. 1981, 58, 123-137.

Fingl, E.;Woodbury, L.A.; Hecht, H.H. Effects of Innervation and Drugs upon Direct Membrane Potentials of Embryonic Chick Myocardium. J. Pharmacol. Exp. Ther. 1952, 104, 103-114.

Fluhler, E.; Burnham, V.G.; Loew, L.M. Spectra, membrane binding, and potentiometric responses of new charge shift probes. Biochemistry 2002, 24, 5749-5755. 
Fujii, S.; Hirota, A.; Kamino, K. Optical recording of development of electrical activity in embryonic chick heart during early phases of cardiogenesis. J. Physiol. 1981, 311, 147-160.

Habib-E-Rasul Mullah, S.; Komuro, R.; Yan, P.; Hayashi, S.; Inaji, M.; Momose-Sato, Y.; Loew, L.M.; Sato, K. Evaluation of Voltage-Sensitive Fluorescence Dyes for Monitoring Neuronal Activity in the Embryonic Central Nervous System. J. Membrain Biol. 2013, 246, 679-688.

Hagiwara, N.; Irisawa, H.; Kameyama, M. Contribution of two types of calcium currents to the pacemaker potentials of rabbit sino-atrial node cells. J. Physiol. 1988, 395, 233-253.

Hamburger, V.; Hamilton, H.L. A series of normal stages in the development of the chick embryo. J. Morphol. 1951, 88, 49-92.

Hill, D.K.; Keynes, R.D. Opacity changes in stimulated nerve. J. Physiol. 1949, 108, 278-281.

Hirota, A.; Sakai, T.; Fujii, S.; Kamino, K. Initial development of conduction pattern of spontaneous action potential in early embryonic precontractile chick heart. Dev. Biol. 1983, 99, 517-523.

Hirota, A.; Fujii, S.; Kamino, K. Optical Monitoring of Spontaneous Electrical Activity of 8-somite Embryonic Chick Heart. Jpn. J. Physiol. 1979, 29, 635-639.

Hoff, E.C.; Kramer, T.C.; DuBois, D.; Patten, B.M. The development of the electrocardiogram of the embryonic heart. Am. Heart J. 1939, 17, 470-488.

Huebsch, N.; Loskill, P.; Mandegar, M.A.; Marks, N.C.; Sheehan, A.S.; Ma, Z.; Mathur, A.; Nguyen, T.N.; Yoo, J.C.; Judge, L.M.; et al. Automated Video-Based Analysis of Contractility and Calcium Flux in Human-Induced Pluripotent Stem Cell-Derived Cardiomyocytes Cultured over Different Spatial Scales. Tissue Eng. Part C Methods 2015, 21, 467-479.

Hyer, J.; Johansen, M.; Prasad, A.; Wessels, A.; Kirby, M.L.; Gourdie, R.G.; Mikawa, T. Induction of Purkinje fiber differentiation by coronary arterialization. Proc. Natl. Acad. Sci. USA 1999, 96, 13214-13218.

Ishii, Y.; Garriock, R.J.; Navetta, A.M.; Coughlin, L.E.; Mikawa, T. BMP Signals Promote Proepicardial Protrusion Necessary for Recruitment of Coronary Vessel and Epicardial Progenitors to the Heart. Dev. Cell 2010, 19, 307-316. 
James, T.N.; Sherf, L.; Fine, G.; Morales, A.R. Comparative ultrastructure of the sinus node in man and dog.Circulation 1966, 34, 139-163.

James, T.N.; Sherf, L. Ultrastructure of the Human Atrioventricular Node. Circulation 1968, 37, 10491070.

Jordan, B.J.; Vogel, S.; Stark, M.R.; Beckstead, R.B. Expression of green fluorescent protein in the chicken using in vivo transfection of the piggyBac transposon. J. Biotechnol. 2014, 173, 86-89.

Joyner, R.W.; van Capelle, F.J. Propagation through electrically coupled cells. How a small SA node drives a large atrium. Biophys. J. 1986, 50, 1157-1164.

Kamino, K. Optical approaches to ontogeny of electrical activity and related functional organization during early heart development. Physiol. Rev. 1991, 71, 53-91.

Kanter, H.L.; Laing, J.G.; Beau, S.L.; Beyer, E.C.; Saffitz, J.E. Distinct patterns of connexin expression in canine Purkinje fibers and ventricular muscle. Circ. Res. 1993, 72, 1124-1131.

Knöpfel, T. Genetically encoded optical indicators for the analysis of neuronal circuits. Nat. Rev. Neurosci. 2012, 13, 687-700.

Lakatta, E.G.; Maltsev, V.A.; Vinogradova, T.M. A Coupled SYSTEM of Intracellular Ca2+ Clocks and Surface Membrane Voltage Clocks Controls the Timekeeping Mechanism of the Heart's Pacemaker. Circ. Res. 2010, 106, 659-673.

Larsen, A.P.; Sciuto, K.J.; Moreno, A.P.; Poelzing, S. The voltage-sensitive dye di-4-ANEPPS slows conduction velocity in isolated guinea pig hearts. Heart Rhythm 2012, 9, 1493-1500.

Loew, L.M.; Bonneville, G.W.; Surow, J. Charge shift optical probes of membrane potential. Theory. Biochemistry 1978, 17, 4065-4071.

Loew, L.M.; Scully, S.; Simpson, L.; Waggoner, A.S. Evidence for a charge-shift electrochromic mechanism in a probe of membrane potential. Nature 1979, 281, 497-499.

Ma, P.; Chan, D.C.; Gu, S.; Watanabe, M.; Jenkins, M.W.; Rollins, A.M. Volumetric optical mapping in early embryonic hearts using light-sheet microscopy. Biomed. Opt. Express 2016, 7, 5120-5129. 
Ma, P.; Wang, Y.T.; Gu, S.; Watanabe, M.; Jenkins, M.W.; Rollins, A.M. Three-dimensional correction of conduction velocity in the embryonic heart using integrated optical mapping and optical coherence tomography. J. Biomed. Opt. 2014, 19, 76004.

Maltsev, V.A.; Lakatta, E.G. Dynamic interactions of an intracellular Ca2+ clock and membrane ion channel clock underlie robust initiation and regulation of cardiac pacemaker function.

Cardiovasc. Res. 2007, 77, 274-284. J. Cardiovasc. Dev. Dis. 2018, 5, 2816 of 19

Mesirca, P.; Torrente, A.G.; Mangoni,M.E. Functional role of voltage gated Ca2+ channels in heart automaticity. Front. Physiol. 2015, 6.

Mikawa, T.; Gourdie, R.G. Pericardial mesoderm generates a population of coronary smooth muscle cells migrating into the heart along with ingrowth of the epicardial organ. Dev. Biol. 1996, 174, 221232.

Mikawa, T.; Borisov, A.; Brown, A.M.C.; Fischman, D.A. Clonal analysis of cardiac morphogenesis in the chicken embryo using a replication-defective retrovirus: I. For mation of the ventricular myocardium. Dev. Dyn. 2006, 193, 11-23.

Nakai, J.; Ohkura, M.; Imoto, K. A high signal-to-noise Ca2+ probe composed of a single green fluorescent protein. Nat. Biotechnol. 2001, 19, 137-141.

Needham, J. Developmental physiology. Annu. Rev. Physiol. 1939, 1, 63-80.

Nussinovitch, U.; Gepstein, L. Optogenetics for in vivo cardiac pacing and resynchronization therapies. Nat. Biotechnol. 2015, 33, 750-754.

Ong, L.L.; Kim, N.; Mima, T.; Cohen-Gould, L.; Mikawa, T. Trabecular Myocytes of the Embryonic Heart Require N-Cadherin for Migratory Unit Identity. Dev. Biol. 1998, 193, 1-9.

Ono, N.; Yamaguchi, T.; Ishikawa, H.; Arakawa, M.; Takahashi, N.; Saikawa, T.; Shimada, T. Morphological varieties of the Purkinje fiber network in mammalian hearts, as revealed by light and electron microscopy. Arch. Histol. Cytol. 2009, 72, 139-149.

Opthof, T.; de Jonge, B.; Mackaay, A.J.; Bleeker, W.K.; Masson-Pévet, M.; Jongsma, H.J.; Bouman, L.N. Functional and morphological organization of the guinea-pig sinoatrial node compared with the rabbit sinoatrial node. J. Mol. Cell. Cardiol. 1985, 17, 549-564. 
Opthof, T.; de Jonge, B.; Jongsma, H.J.; Bouman, L.N. Functional morphology of the mammalian sinoatrial node. Eur. Heart J. 1987, 8, 1249-1259.

Palmer, A.E.; Tsien, R.Y. Measuring calcium signaling using genetically targetable fluorescent indicators. Nat. Protoc. 2006, 1, 1057-1065.

Paredes, R.M.; Etzler, J.C.; Watts, L.T.; Zheng, W.; Lechleiter, J.D. Chemical calcium indicators. Methods 2008, 46, 143-151.

Patten, B.M. Initiation and early changes in the character of the heart beat in vertebrate embryos. Physiol. Rev. 1949, 29, 31-47.

Patten, B.M.; Kramer, T.C. The initiation of contraction in the embryonic chick heart. Am. J. Anat. 1933, 53, 349-375.

Rentschler, S.; Vaidya, D.; Tamaddon, H.; Degenhardt, K.; Sasson, D.; Morley, G.; Jalife, J.; Fishman, G. Visualization and functional characterization of the developing murine cardiac conduction system. Development 2001, 128, 1785-1792.

Rodriguez, E.A.; Campbell, R.E.; Lin, J.Y.; Lin, M.Z.; Miyawaki, A.; Palmer, A.E.; Shu, X.; Zhang, J.; Tsien, R.Y.The Growing and Glowing Toolbox of Fluorescent and Photoactive Proteins. Trends Biochem. Sci. 2017, 42,111-129.

Ross,W.N.; Salzberg, B.M.; Cohen, L.B.; Grinvald, A.; Davila, H.V.;Waggoner, A.S.;Wang, C.H. Changes in absorption, fluorescence, dichroism, and birefringence in stained giant axons: Optical measurement of membrane potential. J. Membrain Biol. 1977, 33, 141-183.

Schaffer, P.; Ahammer, H.; Müller, W.; Koidl, B.; Windisch, H. Di-4-ANEPPS causes photodynamic damage to isolated cardiomyocytes. Pflügers Arch. 1994, 426, 548-551.

Schram, G. Differential Distribution of Cardiac Ion Channel Expression as a Basis for Regional Specialization in Electrical Function. Circ. Res. 2002, 90, 939-950.

Scuderi, G.J.; Butcher, J. Naturally Engineered Maturation of Cardiomyocytes. Front. Cell Dev. Biol. 2017, 5. 
Sedmera, D.; Reckova, M.; Rosengarten, C.; Torres, M.I.; Gourdie, R.G.; Thompson, R.P. Optical Mapping of Electrical Activation in the Developing Heart. Microsc. Microanal. 2005, 11, 209215.

Sedmera, D. Changes in activation sequence of embryonic chick atria correlate with developing myocardial architecture. AJP Heart Circ. Physiol. 2006, 291, H1646-H1652.

Sedmera,D.; Reckova,M.; Bigelow,M.R.; Dealmeida,A.; Stanley, C.P.;Mikawa, T.; Gourdie, R.G.; Thompson, R.P. Developmental transitions in electrical activation patterns in chick embryonic heart. Anat. Rec. 2004, 280A, 1001-1009.

Shiba, Y.; Fernandes, S.; Zhu, W.-Z.; Filice, D.; Muskheli, V.; Kim, J.; Palpant, N.J.; Gantz, J.; Moyes, K.W.; Reinecke, H.; et al. Human ES-cell-derived cardiomyocytes electrically couple and suppress arrhythmias in injured hearts. Nature 2012, 489, 322-325.

Shimada, T.; Kawazato, H.; Yasuda, A.; Ono, N.; Sueda, K. Cytoarchitecture and intercalated disks of the working myocardium and the conduction system in the mammalian heart. Anat. Rec. 2004, 280A, 940-951.

Shinnawi, R.; Huber, I.; Maizels, L.; Shaheen, N.; Gepstein, A.; Arbel, G.; Tijsen, A.J.; Gepstein, L. Monitoring Human-Induced Pluripotent Stem Cell-Derived Cardiomyocytes with Genetically Encoded Calcium and Voltage Fluorescent Reporters. Stem Cell Rep. 2015, 5, 582-596.

Smith, A.S.T.; Macadangdang, J.; Leung,W.; Laflamme, M.A.; Kim, D.-H. Human iPSC-derived cardiomyocytes and tissue engineering strategies for disease modeling and drug screening. Biotechnol. Adv. 2017, 35, 77-94.

Sperelakis, N.; Pappano, A.J. Physiology and pharmacology of developing heart cells. Pharmacol. Ther. $1983,22,1-39$.

Sperelakis, N.; Shigenobu, K. Changes in Membrane Properties of Chick Embryonic Hearts during Development. J. Gen. Physiol 1972, 60, 430-453.

Takebayashi-Suzuki, K.; Yanagisawa, M.; Gourdie, R.G.; Kanzawa, N.; Mikawa, T. In vivo induction of cardiac Purkinje fiber differentiation by coexpression of preproendothelin-1 and endothelin converting enzyme-1. Development 2000, 127, 3523-3532. 
Tallini, Y.N.; Ohkura, M.; Choi, B.-R.; Ji, G.; Imoto, K.; Doran, R.; Lee, J.; Plan, P.;Wilson, J.; Xin, H.B.; et al. Imaging cellular signals in the heart in vivo: Cardiac expression of the high-signal $\mathrm{Ca} 2+$ indicator GCaMP2. Proc. Natl. Acad. Sci. USA 2006, 103, 4753-4758.

Tian, L.Monitoring activity in neural circuits with genetically encoded indicators. Front. Mol. Neurosci. $2014,7$.

Tsien, R.Y. New calcium indicators and buffers with high selectivity against magnesium and protons: Design, synthesis, and properties of prototype structures. Biochemistry 1980, 19, 2396-2404.

Tsien, R.Y. Fluorescent probes of cell signaling. Annu. Rev. Neurosci. 1989, 12, 227-253.

Tyser, R.C.; Miranda, A.M.; Chen, C.-M.; Davidson, S.M.; Srinivas, S.; Riley, P.R. Calcium handling precedes cardiac differentiation to initiate the first heartbeat. eLife 2016, 5, e17113.

Tzatzalos, E.; Abilez, O.J.; Shukla, P.; Wu, J.C. Engineered heart tissues and induced pluripotent stem cells:Macro- and microstructures for disease modeling, drug screening, and translational studies. Adv. Drug Deliv. Rev. 2016, 96, 234-244.

Vaidyanathan, R.; O’Connell, R.P.; Deo, M.; Milstein, M.L.; Furspan, P.; Herron, T.J.; Pandit, S.V.; Musa, H.; Berenfeld, O.; Jalife, J.; et al. The ionic bases of the action potential in isolated mouse cardiac Purkinje cell. Heart Rhythm 2013, 10, 80-87.

Van Mierop, L.H. Location of pacemaker in chick embryo heart at the time of initiation of heartbeat. Am. J. Physiol. 1967, 212, 407-415.

Veerman, C.C.; Kosmidis, G.; Mummery, C.L.; Casini, S.; Verkerk, A.O.; Bellin, M. Immaturity of human stem-cell-derived cardiomyocytes in culture: Fatal flaw or soluble problem? Stem Cells Dev. 2015, 24, 1035-1052.

Verkerk, A.O.; Wilders, R.; van Borren, M.M.G.J.; Peters, R.J.G.; Broekhuis, E.; Lam, K.; Coronel, R.; de Bakker, J.M.T.; Tan, H.L. Pacemaker current (If) in the human sinoatrial node. Eur. Heart J. 2007, 28, 2472-2478.

Virágh, S.; Challice, C.E. The development of the conduction system in the mouse embryo heart: IV. Differentiation of the atrioventricular conduction system. Dev. Biol. 1982, 89, 25-40. 
Wang, Y.T.; Gu, S.; Ma, P.; Watanabe, M.; Rollins, A.M.; Jenkins, M.W. Optical stimulation enables paced electrophysiological studies in embryonic hearts. Biomed. Opt. Express 2014, 5, 1000 1013. J. Cardiovasc. Dev. Dis. 2018, 5, 2819 of 19

Weber, M.; Huisken, J. In vivo imaging of cardiac development and function in zebrafish using light sheet microscopy. Swiss Med. Wkly. 2015, 145, w14227.

Weidmann, S. The effect of the cardiac membrane potential on the rapid availability of the sodiumcarrying system. J. Physiol. 1955, 127, 213-224.

Xu, Y.; Zou, P.; Cohen, A.E. ScienceDirect Voltage imaging with genetically encoded indicators. Curr. Opin. Chem. Biol. 2017, 39, 1-10.

Yelbuz, T.M. Optical Coherence Tomography: A New High-Resolution Imaging Technology to Study Cardiac Development in Chick Embryos. Circulation 2002, 106, 2771-2774.

Zhang, S.S.; Kim, K.H.; Rosen, A.; Smyth, J.W.; Sakuma, R.; Delgado-Olguín, P.; Davis, M.; Chi, N.C.; Puviindran, V.; Gaborit, N.; et al. Iroquois homeobox gene 3 establishes fast conduction in the cardiac His-Purkinje network. Proc. Natl. Acad. Sci. USA 2011, 108, 13576-13581. 


\section{CHAPTER 3: ADHERENS JUNCTION ENGAGEMENT REGULATES FUNCTIONAL PATTERNING OF THE CARDIAC PACEMAKER CELL LINEAGE ${ }^{2}$}

\section{Introduction}

A network of cardiac pacemaker cells (CPCs) located in the sinoatrial node (SAN) autonomously initiate the electrical impulses that trigger rhythmic cardiac contraction. CPCs represent only a fraction of the total cellular volume of the heart (Joyner and van Capelle, 1986; Kapoor et al., 2012; Unudurthi et al., 2014), yet they must generate sufficient ionic current to activate the entire working myocardium (WM). It has long been recognized that the low proportion of CPCs relative to the WM represents a significant obstacle to rhythmic impulse generation and propagation (Joyner and van Capelle 1986, Unudurthi, Wolf et al. 2014, Grijalva, Gu et al. 2019). CPCs need to depolarize while in electrical communication with a large volume of cells that are actively maintaining a negative membrane potential. In theory, this ionic configuration would be predicted to elicit subthreshold current flows from CPCs to WM, delaying or preventing the ability of CPCs to build up enough local charge to initiate an action potential (Joyner and van Capelle 1986, Kirchhof, Bonke et al. 1987, Fast and Kléber 1995, Rohr, Kucera et al. 1997, Rohr, Kucera et al. 1998, Kleber and Rudy 2004, Nikolaidou, Aslanidi et al. 2012, Unudurthi, Wolf et al. 2014). The ability of CPCs to escape such inhibitory forces is a topic of fundamental interest to our overall understanding of cardiac electrophysiology, however, the mechanisms that dictate how CPCs regulate the extent of their electrogenic interaction with the WM remain poorly understood. Previously, we identified that CPCs differentiate relatively late during cardiac morphogenesis and integrate into an already electrically active

\footnotetext{
2 This chapter previously appeared as an article in Developmental Cell. The original citation is as follows: Thomas K, Henley T, Bai X, Taylor J, Costello MJ, Bressan M. Adherens Junction Engagement Regulates Functional Patterning of the Cardiac Pacemaker Cell Lineage. Developmental Cell. 05/2021; 56(10):1498-1511.e7. doi: 10.1016/j.devcel.2021.04.004
} 

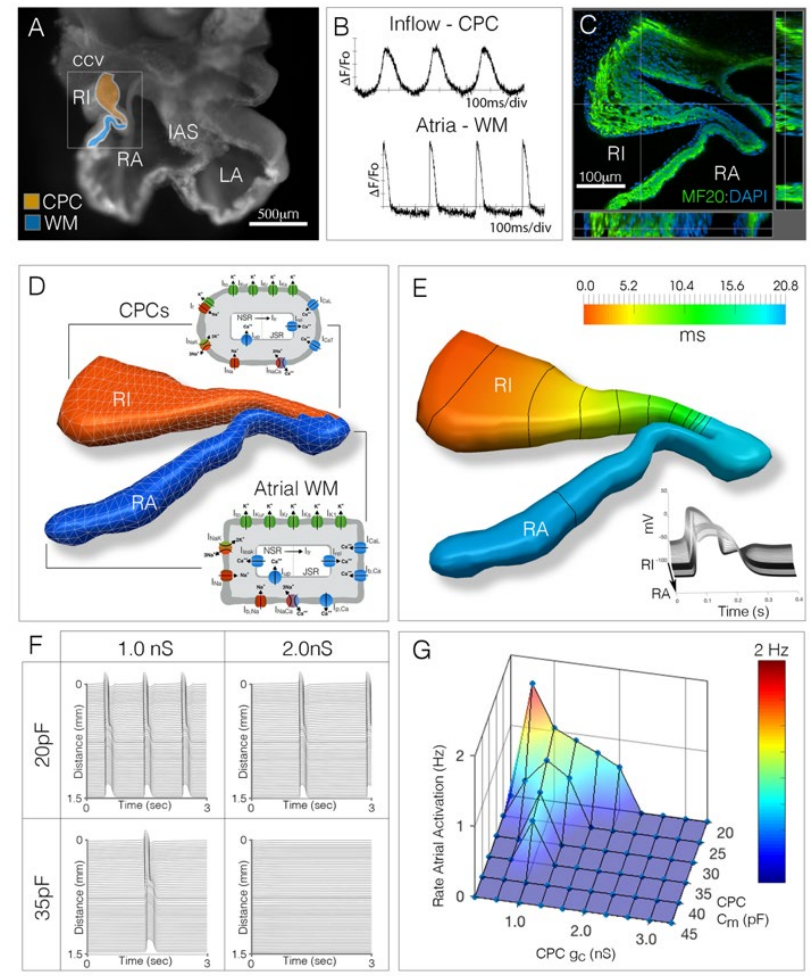

Figure 9. Mathematical simulation of electrical interactions in the embryonic heart cellular parameters critical for successful pacemaking.

A) Cardiac slice preparation for live-imaging of electrical activity in the embryonic chick heart. Orange region denotes the right inflow (RI) myocardium which displays cardiac pacemaker cell (CPC)-like electrical behavior, blue region denotes adjacent right atrial (RA) muscle with working myocardium (WM)-like physiological features. IAS interatrial septum, ccv - common cardinal vein. Scale bare $-500 \mathrm{~mm}$. B) Electrical activity recorded from inflow (orange region from "A") and right atria (blue region from "A"). C) $300 \mu \mathrm{m}$ thick section through the CPC/WM interface, tissue was stained with the muscle marker MF20 (green) and DAPI (blue). Scale bar - $100 \mathrm{~mm}$ D) Tissue geometry from "C" was segmented and the region of the tissue that displayed CPC physiological activity (from "A,B") was simulated as containing cells utilizing CPC ionic behavior (orange), while tissue regions that displayed atrial-like physiological features (from "A,B") were simulated as containing atrial WM ionic features (blue region). E) Simulation of impulse generation and propagation through the segmented geometry from "D." Isochronal map indicates initial activation in the right inflow (red) and propagation into the atrial myocardium (blue). Lines denote propagation at $5 \mathrm{~ms} / \mathrm{div}$. Inset shows action potential shapes as the impulse propagates from right inflow to right atria. F) Changes to $\mathrm{CPC}$ cellular parameters, such as cell size (equivalent to cell capacitance, $\mathrm{pF}$ ) or coupling conductance $(\mathrm{nS})$, directly impact rate of activity. G) Sensitivity analysis comparing variations in CPC cell size and conductance $\left(\mathrm{C}_{\mathrm{m}}[\mathrm{pF}]\right.$ vs $\left.g_{c}(\mathrm{nS})\right)$ on rate of activity. 


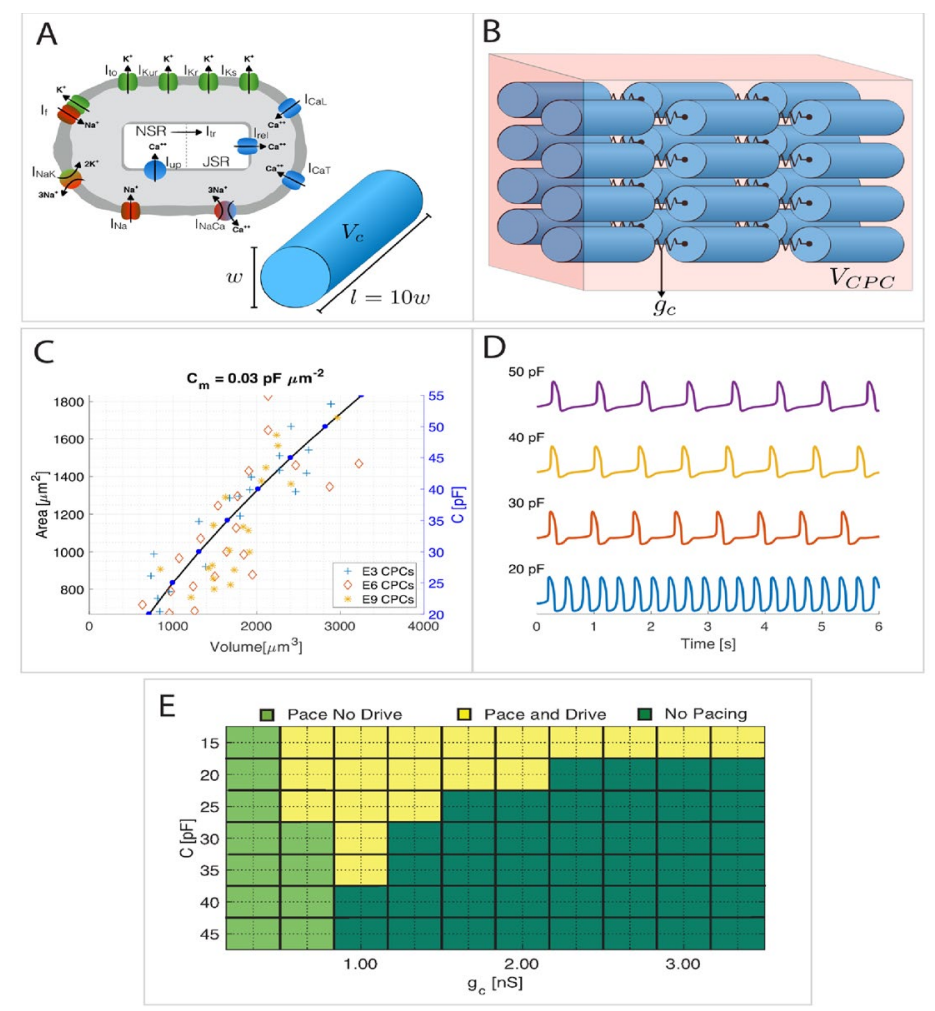

Figure 10 Computational model of the CPC activation.

A) Schematic diagram of the Fabbri et al. (Fabbri et al., 2017) human cell model. A single CPC cell is represented by a cylinder width $w$ and length $l=10 \mathrm{w}$. The cell volume $V_{\mathrm{c}}$ influences the total junctional and network sarcoplasmic reticulum Calcium fluxes. B) Schematic representation of the three-dimensional structure of the tissue. The volume $V_{\mathrm{CPC}}$ containing the CPCs and the number $n_{\mathrm{CPC}}$ of CPCs determine the volume fraction of extracellular space $\alpha$ and the membrane surface to tissue volume ratio $\chi$. C) The specific membrane capacitance $C_{\mathrm{m}}$ is adjusted to $0.03 \mathrm{pF} \mu \mathrm{m}-2$ to fit the cellular area/volume relationship at various stages of development, leading to total capacitances varying between 20 and $55 \mathrm{pF}$. D) Changes in the action potential computed using the Fabbri et al. [6] cell model for selected values of the cell capacitance. Changes in the cell capacitance are linked to the cell dimension and influence the subcellular calcium handling of the model. E) Categorization of the behavior of the CPCs-WM coupled three-dimensional simulations as a function of cell capacitance $C$ and coupling conductance $g$ c. For small values of $g_{c}$, although the CPCs are able to trigger an action potential, the coupling is too small to stimulate successfully the working myocardium (CPCs pace but do not drive the WM). For larger values of $g_{c}$ the CPCs can stimulate the WM successfully provided the cell capacitance is small enough (CPCs pace and drive the WM). For large values of $g_{c}$, and $C$, the coupling between the CPCs and the WM is too strong and there is no electrical activity in the system (CPCs do not pace).

embryonic heart (Bressan, Liu et al. 2013). Thus, we hypothesized that the mechanisms required for CPCs to overcome WM-mediated electrical suppression should rapidly manifest shortly after they are added to the heart.

In this study we have now identified a critical relationship between two parameters that significantly impact CPC electrical excitability during cardiogenesis: cell size regulation and cell-cell 
coupling. Importantly, our data indicate that these features are related to one another, both in terms of their predicted influence on CPC function and in regard to their molecular regulation. Furthermore, we show that the cardiac adherens junction (AJ) plays a previously unrecognized role as a molecular regulator of CPC structural patterning. Collectively, these findings have uncovered a cellular mechanicsbased model for CPC developmental maturation that defines specific cytoarchitectural features that support CPC function and identifies how these characteristics are patterned and maintained in the forming heart.

\section{Results}

$\underline{\text { Computational modeling of the embryonic } \mathrm{CPC} / \text { atrial interface predicts that an interplay between }}$ $\underline{\text { membrane capacitance and intercellular conductance governs successful pacemaking activity }}$

To explore the cellular parameters that may influence CPC activity and/or excitability, we generated a computational model of the CPC/WM electrical interface based on the geometry of the embryonic heart and probed for critical features that lead to SAN failure (Figures 9 and 10). Herein, 300um thick sections of the embryonic SAN/atria were imaged, digitally segmented, and reconstructed in silico (Figures 9A-9D). CPCs and atrial ionic currents were modeled based on Fabbri et al. (Fabbri, Fantini et al. 2017) and Courtemanche et al.(Courtemanche, Ramirez et al. 1998) respectively. Modeled CPCs were assigned to the region of the myocardium that our voltage imaging demonstrated displayed pacemaking activity and expressed the CPC functional marker, Hcn4 (Bressan et al 2018) (Figures 9A9D). Voltage imaging from live cardiac slices were used to qualitatively assess fundamental features of the modeled data including action potential kinetics, conduction velocities, and activation patterns to ensure that computational simulations replicated basic features of the embryonic SAN/Atrial interface (Figure 9E, see Methods).

While constructing the computational model, physiological and structural features such as cellular capacitance $(\mathrm{Cm})$, membrane surface to tissue volume ratio $(\mathrm{\chi})$, current densities, and the tissue conductivity $(\sigma)$ were modified to investigate the resultant effects on CPC activity (see Methods). In 
agreement with previous modeling studies, our data indicated that coupling conductance $\left(g_{c}\right)$ plays a critical role in maintaining rate of activity and 1:1 CPC/WM entrainment (Figures 9F and 10E), with $g_{c}$ needing to be held at low values to prevent WM-mediated suppression of CPC function (Joyner and van Capelle 1986, Zhang, Holden et al. 2000, Glynn, Onal et al. 2014, Inada, Zhang et al. 2014). In addition to $g_{c}$, our data also highlighted that membrane capacitance $\left(C_{m}\right)$ dramatically influenced behavior in the simulations. Increasing CPC $C_{m}$ decreased the rate of CPC oscillation and blocked the ability of CPCs to drive the atria (Figures 9F and 10D). Given the predicted influence that both $g_{c}$ and $C_{m}$ displayed in our computational modeling, we simultaneously altered the ranges of both parameters. In general, lowering $C_{m}$ increased the ranges of $g_{c}$ that could be tolerated before pacemaking failure with a reciprocal effect in which lowering $g_{c}$ expanded the values of $C_{m}$ that allowed for effective pacing (Figures 9G, 10E). Importantly, these data demonstrate that both $g_{c}$ and $C_{m}$ may influence pacemaking activity in the embryonic heart and indicate that changes in one may affect the range of values that are possible in the other. 

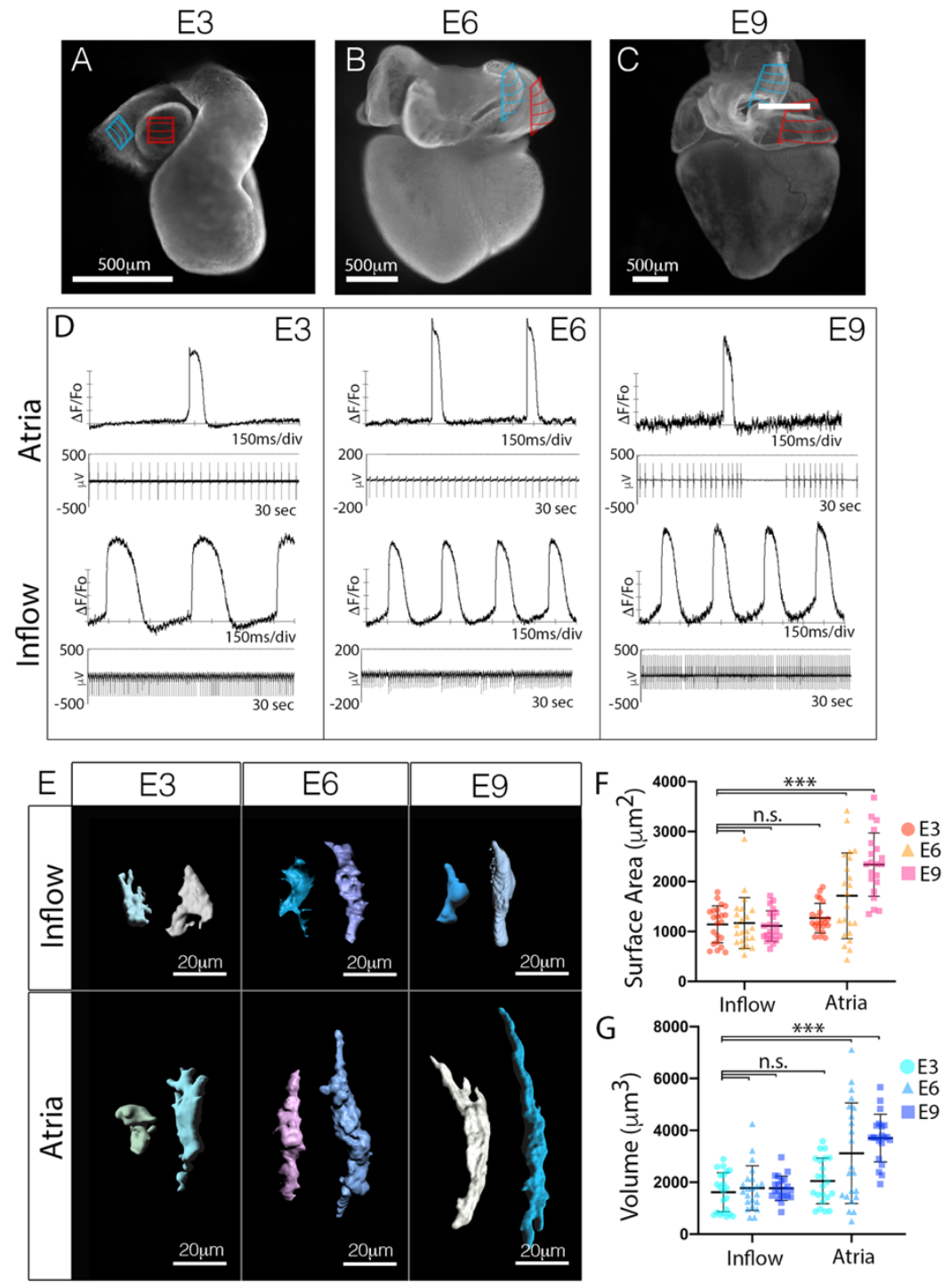

Figure 11. Cell size is differentially regulated between the CPC and WM lineages during cardiac morphogenesis.

A-C) Whole mount staining for the muscle marker MF20 at embryonic day 3 (A), embryonic day 6 (B), and embryonic day 9 (C). The location of the CPCs within the inflow myocardium is indicated by the blue hatched area, the atrial WM used for size analysis is indicated by the red hatched area. D) Voltage sensitive dye-based (top) and microelectrode array (MEA) (bottom) recordings of electrical activity from isolated segments of tissues (blue and red regions from (A-C)). Scale bar $-500 \mathrm{~mm}$. E) Volumetric reconstruction of CPCs from the inflow and atrial WM at different stages of cardiac morphogenesis (See also Figure 10). F) Quantification of surface area of CPCs from the inflow and atrial WM at different stages of cardiac morphogenesis $(\mathrm{n}=129)$. G) Quantification of cell volume of CPCs from the inflow and atrial WM at different stages of cardiac morphogenesis $(\mathrm{n}=129)$. Mean $+/-\mathrm{SD}$ are indicated by line and bars for $\mathrm{F}$ and $\mathrm{G}$. 

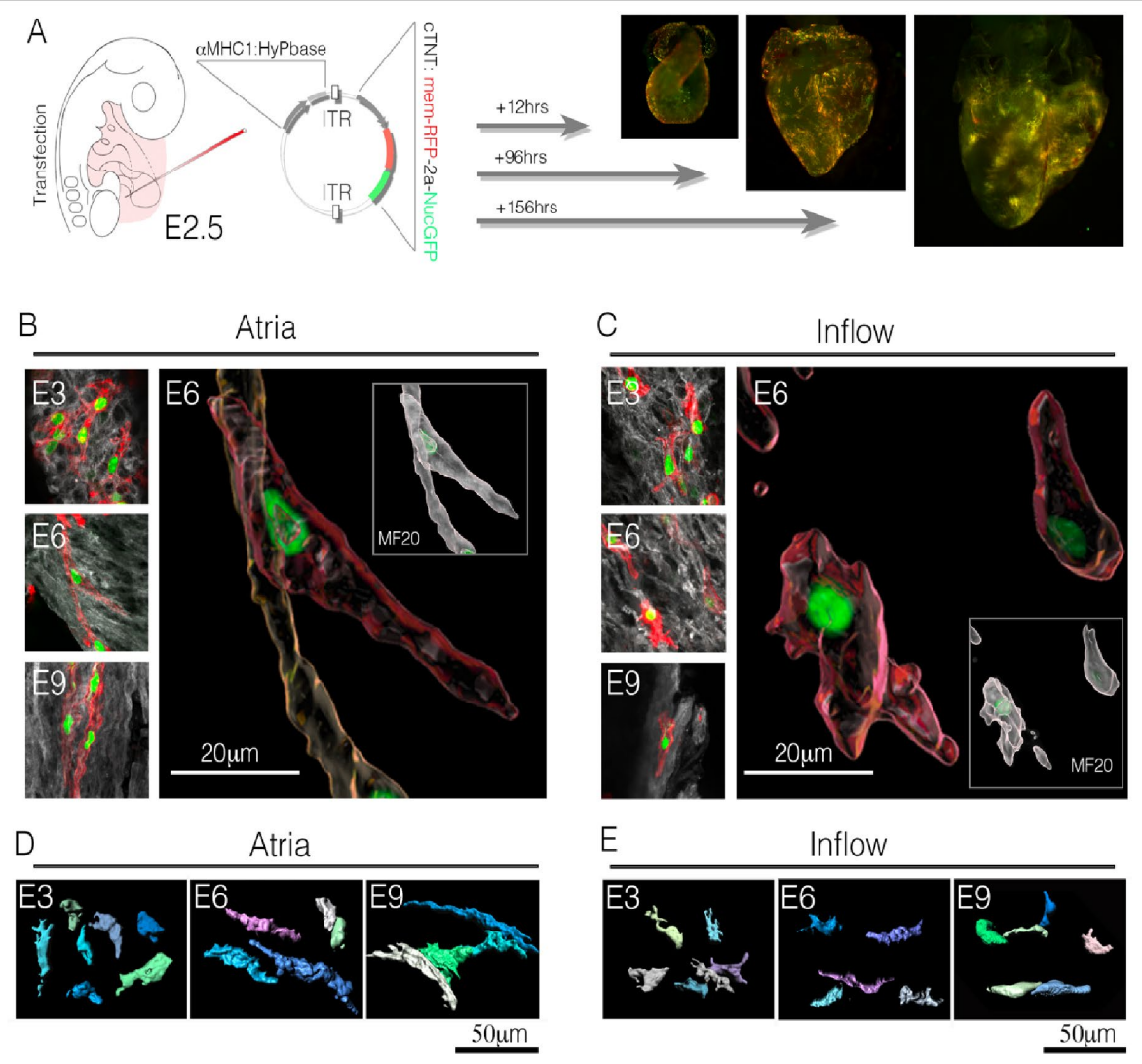

Figure 12. In situ cell size determination.

A) Diagram of targeted in vivo transfection protocol. At embryonic day 2.5 (E2.5), lipofectamine/DNA were microinjected into the pericardial space surrounding the heart. Plasmid DNA contained two expression cassettes: i) the alpha Myosin Heavy Chain 1 promoter driving the high fidelity PiggyBac transposase (HyPBase) and ii) the chick cardiac Troponin promoter driving palmitoylated TagRFP-2A-H2bEGFP flanked by transposable inverted terminal repeats (ITRs). This results in reporter expression/integration targeted to developing cardiomyocytes. Following transfection, embryos were incubated to desired stages and individual transfected cells were imaged. B) Imaging of atrial cells at E3, E6, and E9. Volumetric reconstruction of two of cells from E6 are shown. Scale bar $-20 \mu \mathrm{m}$. Inset displays MF20 staining digitally isolated from the reconstructed cells confirming that these cells are myocytes. C) As in "B" for inflow cells. F) Volumetric reconstructions of atrial cells at E3, E6, and E9. Scale bar - $50 \mu \mathrm{m}$. G) Volumetric reconstructions of inflow cells at E3, E6, and E9. Scale bar $-50 \mu \mathrm{m}$.

Data from the above computer simulations are in agreement with the known cellular architecture of mature CPCs. Total membrane capacitance is proportional to cell size (Satoh, Delbridge et al. 1996), and adult CPCs are substantially smaller and have lower $C_{m}$ than their WM counterparts (Masson-Pevet, Bleeker et al. 1979, Honjo, Boyett et al. 1996, Miyamoto, Zhang et al. 2002, Shimada, Kawazato et al. 2004, Lyashkov, Juhaszova et al. 2007, Jones, Yamamoto et al. 2008). Additionally, $g_{c}$ is believed to be determined by gap junction-mediated coupling and mature CPCs have few gap junctions (GJs)(Forbes 
and Sperelakis 1985, Boyett, Honjo et al. 2000, Honjo, Boyett et al. 2002, Miyamoto, Zhang et al. 2002, Shimada, Kawazato et al. 2004). Therefore, our simulations identified known cellular features of the adult heart as potentially important for embryonic CPC electrical activity and highlighted that developmental events upstream of cell size regulation and GJ coupling may be critical for proper cardiac pacemaking. $\underline{\text { CPCs structurally diverge from the WM after becoming electrically active }}$

Motivated by the computational model predictions, we conducted a developmental survey of cellular architecture to determine whether CPCs regulate their morphology in a manner consistent with our simulations.

Initially, we confirmed the location of CPCs at various stages of heart development by monitoring electrical activity using both voltage imaging and micro-electrode array recordings (MEA). Consistent with previous work (Bressan, Liu et al. 2013, Bressan, Henley et al. 2018), our data indicated that CPCs were present in the right inflow myocardium from embryonic day 3 (E3) through E9 of avian cardiac morphogenesis (Figure 11A-D). To evaluate regional patterning of cell size, we generated mosaic hearts using in vivo transfection of a transposable expression construct encoding a palmitoylated membrane RFP reporter and GFP nuclear reporter (Figure 12A) (Goudy, Henley et al. 2019) and threedimensional renderings of individual myocytes were generated to quantify cellular geometry (Figures 12B-12E). At E3, no statistical differences in cell volume or surface area were noted between CPCs and atrial WM (Figures 11E-11G). However, following three additional days of incubation, statistically significant differences in cell structure began to emerge (Figures 11E-11G). At E6, CPCs retained the structural characteristics present in E3 myocytes, while atrial WM had increased in both surface area and volume. By the completion of cardiac morphogenesis at E9, CPCs had still not changed in their basic structural features compared to their E3 counterparts. In contrast, atrial WM had increased both their surface area and volumes by factors of 2.1 and 2.4-fold respectively (Figures 11E-11G). These data demonstrate that CPCs and atrial WM initially share similar cytoarchitectural features, however, as cardiac morphogenesis proceeds, WM increase in size while CPCs do not. 


\section{CPCs lose the ability to form AJ as they diverge from the WM}

Having determined a developmental window during which CPCs and WM structurally diverge, we sought to identify mechanisms regulating cell size. Transmission electron microscopy (TEM) was used to view the ultrastructure of CPCs vs WM at stages when differences in cell structure first became apparent (E6). Consistent with previous data from adult vertebrates (Masson-Pevet, Bleeker et al. 1984, Opthof 1988, Lu, James et al. 1993), CPCs located in the forming SAN (Figure 11A) (Bressan, Liu et al. 2013, Bressan, Henley et al. 2018) contained fewer myofibrils than the atrial WM (Figures 13A-13F). Within the atrial WM, large electron dense structures were noted spanning opposing plasma membranes (Figure 13A-13C). These structures averaged $\sim 600 \square \mathrm{m}$ in length and were tethered to collections of actin filaments (Figures 13A-13C, 13G), indicative of classic AJs (Yonemura, Itoh et al. 1995, Gutstein, Liu et al. 2003, Merkel, Li et al. 2019). Of note, CPCs displayed long stretches of membrane between adjacent cells with few or no AJ-like structures. When potential AJs were observed, they appeared shorter than in the WM and had few actin fibers associated with them (Figures 13D-13F). These clear differences in cellcell adhesion were largely restricted to AJs, as our TEM imaging noted that desmosomes were detected at similar abundance among CPCs and atrial WM (Figure 13C and 13F) which is in agreement with studies from the adult SAN (Mezzano, Liang et al. 2016, Mavroidis, Athanasiadis et al. 2020).

The lack of AJs among CPCs was of immediate interest given the known role for these structures in conveying the biomechanical signals that induce hypertrophic growth in a variety of cell types including myocytes (Janssens, Goossens et al. 2001, Luo, Ferreira-Cornwell et al. 2001, Luo 2003, Chopra, Tabdanov et al. 2011, Li, Goossens et al. 2012, Wickline, Dale et al. 2016, Vite, Zhang et al. 2018). Thus, we verified our TEM studies by examining the localization of proteins associated with formation of the cardiac AJ via immunostaining for N-cadherin (N-Cad), Beta-Catenin ( $\beta$-cat), and alphaT-Catenin ( $\alpha \mathrm{T}$-cat) at E3, E6, and E9. Interestingly at E3, when CPCs and atrial WM display similar structural characteristics (Figure 11E-G), no regional differences in N-Cad, $\beta$-cat, or $\alpha \mathrm{T}$-cat protein localization were noted (Figures 13H,13I,14A, and 14B). However, by E6 and continuing through E9, AJ 
proteins were largely absent from the inflow cells, while they were clearly present at cell borders in the atrial WM (Figures 13H,13I,14A, and 14B). At stages when N-cad protein levels were diminished in CPCs, mRNA transcript levels were similar with those detected in the WM (Figures 14C and 14D). To confirm these data in a mammalian embryo, we examined sections through an E14.5 embryonic mouse heart. Consistent with our data from the chick, HCN4-positive CPCs in the mouse embryonic SAN showed diminished staining for N-Cad and $\alpha \mathrm{T}$-cat when compared with the atrial WM (Figures 14E,14F). To confirm that diminished immunoreactivity was not due to variations in section plane we cut $200 \square \mathrm{m}$ thick sections through the inflow and atria of E6 hearts and examined N-Cad distribution (Figures 13J13M). Line scans through these sections confirmed that N-Cad was largely absent among CPCs.

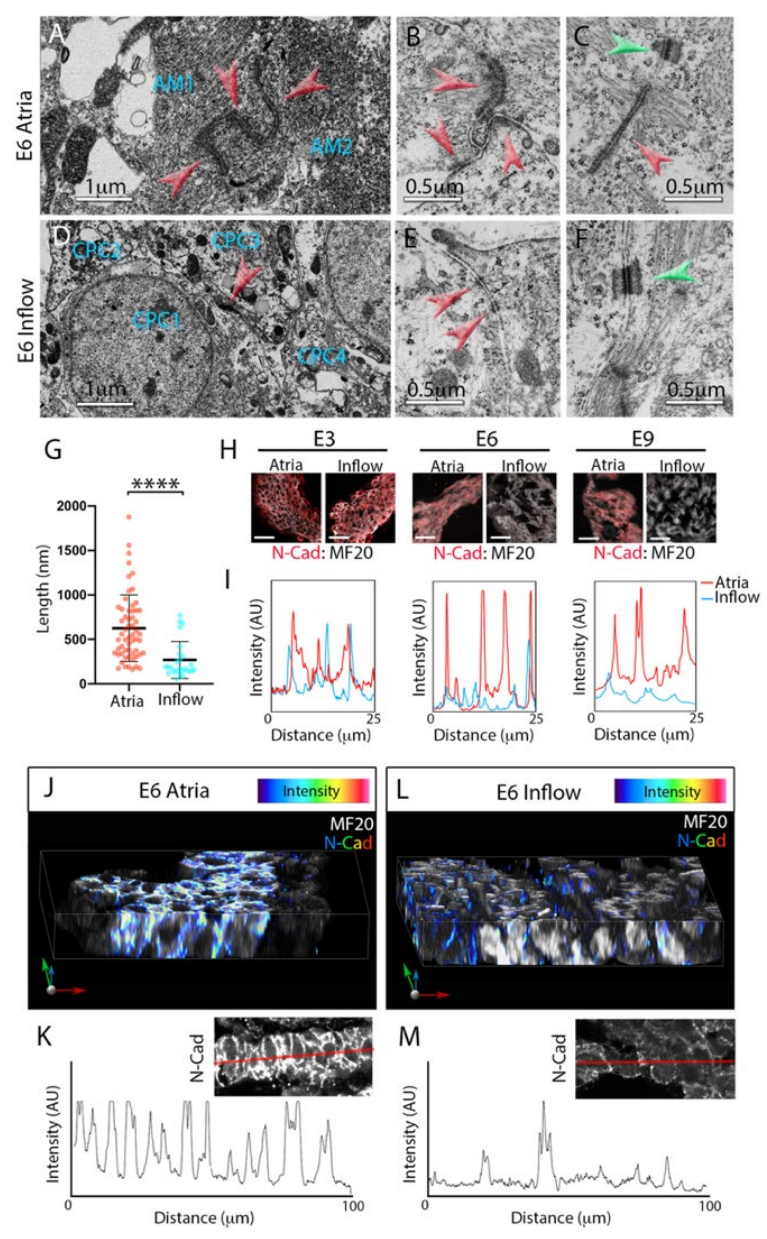


Figure 13. CPCs uniquely lose the capacity to form adherens junctions as they structurally diverge from the working myocardium.

A) Transmission electron microscope (TEM) image of two atrial myocytes (AM1 and AM2). Red arrowheads indicate a series of adherens junctions (AJs) between the two myocytes. B) Higher magnification image of a series of AJs between three atrial myocytes (red arrowheads), these structures are tethered to large bundles of actin filaments. C) Within the atrial WM, AJs are often associated with nearby desmosomes (green arrowhead). D) TEM image of four CPCs (CPC1-4) located within the inflow myocardium. These cells have low sarcomere density consistent with adult CPCs. Note: only one junction is present between the four cells (red arrowhead). E) Higher magnification image of membrane interface between two inflow cells, small electron dense structures that are not tethered to actin are present (red arrowheads). F) Typical desmosome present between inflow myocytes (green arrowhead). G) Quantification of measured AJs length based on TEM images ( $\mathrm{n}=89)$. Mean $+/-\mathrm{SD}$ are indicated by line and bars. H) Immunolocalization of the AJ protein, N-cadherin (N-Cad, red), within E3, E6, and E9 atrial and inflow myocardium. Sections are counterstained with MF20 (white). Scale bar $-25 \mathrm{~mm}$. I) Line scan profile comparing intensity of atrial (red trace) and inflow (blue trace) N-Cad staining at E3, E6, and E9. J) Reconstruction of a $30 \mu \mathrm{m}$ Z-stack of atrial WM taken from a $200 \mu \mathrm{m}$ thick vibratome section. N-Cad intensity is color coded. K) Line scan of N-Cad staining taken from a $200 \mathrm{~mm}$ section of atrial tissue. Inset shows N-Cad staining, red line indicates position of the line scan. $\mathbf{L}, \mathbf{M})$ As in $(\mathrm{K}, \mathrm{L})$ for inflow myocardium.
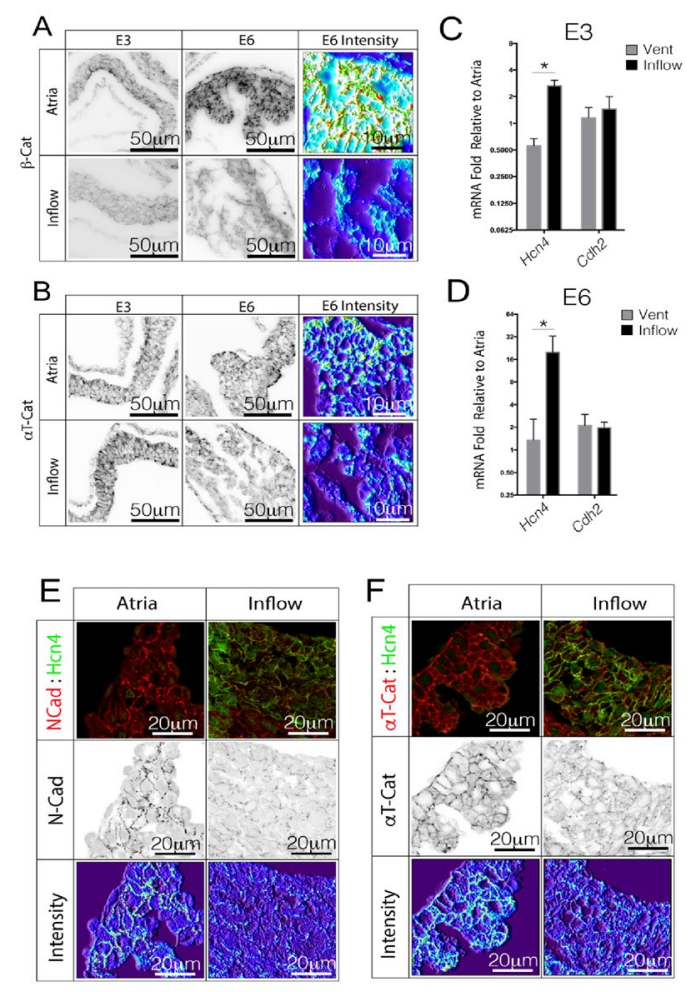

Figure 14. AJ protein localization in the forming SAN.

A) Immunostaining for b-Catenin in atrial and in flow myocardium at E3 and E6. Gray scale images are inverted. E6 images are also shown color coded for staining intensity. Scale bars $-50 \mu \mathrm{m}$. B) As in (A), for immunostaining against aTCatenin. C) Quantification of real-time PCR for CPC marker Hcn4 and AJ protein $\mathrm{Cdh} 2$ (coding for N-cadherin comparing expression levels in E3 inflow and ventricular myocardium relative to atrial levels ( $n=3$ biological replicates, consisting of 3 pooled samples). Mean $+/$ - SD. D) As in (C), for E6 samples. E) Immunostaining against $\mathrm{N}-\mathrm{Cad}$ in E14.5 mouse atrial and Inflow (HCN4+) myocardium. Scale bars $-20 \mu \mathrm{m}$. F) As in (E), immunostaining against aT-Catenin. 
Inhibition of the cardiac AJ converts atrial WM to a CPC-like morphology

To test whether diminished AJ formation alone could generate myocytes with CPC-like structural characteristics, we blocked AJ formation in the WM. We developed two independent shRNAs against NCad (Goudy, Henley et al. 2019) and generated a mutated N-Cad expression construct lacking the extracellular domain required for intercellular binding (N-cad $\Delta 1$ 12-624) (Ong, Kim et al. 1998). When transfected into primary embryonic WM, both shRNAs and the N-cad $\Delta 112-624$ construct diminished cell-cell contacts and AJ-formation in cultured primary embryonic WM (Figure 16). The N-cad $\Delta 112-624$ construct was slightly more effective at disrupting AJ formation in vitro (Figures 16A and 16C), therefore, we used this construct to evaluate the role of AJ formation in cell size regulation in vivo. Following transfection at E2.5 and incubation to E9 (Figure 15A), atrial WM expressing N-cad $\Delta 112-624$ displayed a $29.1 \%$ decrease in surface area and a $41.0 \%$ decrease in volume when compared to control transfected cells (Figures 15C-15H), resulting in WM with CPC-like morphologies in vivo.

\section{Force-mediated AJ engagement induces a WM-like phenotype in CPCs}

We next tested whether forcing AJ engagement would alter the phenotype of embryonic CPCs. At E2.5, hearts were transfected with a construct expressing a membrane localized RFP (palmTagRFP). Inflow and atrial WM were then isolated at E6. Fluorescent in situ hybridization (RNAscope) against the CPC marker gene Hcn4 was used to confirm isolated inflow cells showed molecular characteristics of CPCs (Figures 18A-18C). Dissociated inflow and atrial cells were then cultured for 72 hours on polyacrylamide gels of varying stiffness $(4 \mathrm{kPa}-60 \mathrm{kPa})$ coated with recombinant $\mathrm{N}-\mathrm{Cad}$. In agreement with previous studies (Chopra, Tabdanov et al. 2011), atrial WM displayed N-Cad-mediated, stiffnessdependent, regulation of morphology. On relatively compliant gels $(4 \mathrm{kPa})$ atrial cells were small and round (Figure 17A). However, atrial WM cultured on stiffer gels $(60 \mathrm{kPa})$ exhibited increases in both surface area and volume (Figures 17A-17C). Importantly, CPCs plated on N-Cad coated polyacrylamide gels displayed morphological changes similar to the atrial WM. On 4kPa gels, CPCs had an average surface area of $1697+/-931.2 \square \mathrm{m}^{2}$ and an average volume of $\sim 2217.5+/-1384.7 \square \mathrm{m}^{3}$. However, when 
CPCs were plated on $60 \mathrm{kPa}$ gels coated with $\mathrm{N}-\mathrm{Cad}$ both surface area and volume increased by 1.48 -fold and 1.84-fold respectively (Figures 17A-17C). These data indicate that WM and CPCs displayed similar size characteristics when presented with similar levels of force-dependent, AJ-engagement. Importantly, size changes among CPCs and atrial myocytes converged towards common characteristics in these studies and did not simply scale based on their initial properties at the time of isolation (CPCs were smaller than atrial cells at the time of isolation), indicating that cell size regulation in both the embryonic WM and CPC lineages is plastic and responsive to local biomechanical cues.

To test whether AJ formation altered CPC function, cells from the above analysis were liveimaged and calcium transient activity was monitored to assay the rate and rhythmicity of electrical activity. Regardless of N-Cad substrate stiffness, atrial WM displayed similar functional characteristics, with impulse generation being relatively arrhythmic and spontaneous depolarization occurring at an average rate of $0.46 \mathrm{~Hz}$ (Figure 17D-17F). CPCs cultured on soft N-Cad gels were 5.0-fold more rhythmic than WM and were 2.5-fold faster in their rate of activity (Figures 17E-17F). However, the rate and rhythmicity of CPCs significantly dropped when the cells were subjected to stiffer N-Cad coated substrates (Figure 17F,17G), with a weak positive correlation between surface area/volume ratio and rate of activity apparent across both conditions $\left(\mathrm{R}^{2}=0.21\right.$, Figure $\left.17 \mathrm{H}\right)$.

The above studies are in agreement with our computational modeling that suggested that increasing surface area should negatively impact CPC activity. However, these studies could not rule out that the dominant effect on our cultured cells was a response to substrate stiffness as opposed to changes in cell size. To address this, we plated cells on intermediate $14 \mathrm{kPa}$ gels coated with fibronectin (FN) or NCad and monitored cell size and activity at 24 and 72 hrs. of culture. On both FN and N-Cad, CPCs were relatively small at 24 hrs. but increased by 4.9 and 2.8 -fold respectively by $72 \mathrm{hrs}$ (Figures $18 \mathrm{~F}, 18 \mathrm{G}$ ). The larger CPCs at 72 hrs. of culture decreased rate of activity on both FN and N-Cad despite the fact that substrate stiffness was held constant throughout the analysis (Figure 18G). In addition, we plated CPCs on $50 \mathrm{kPa}$ gels with micropatterned wells to constrain their size on a stiff substrate. CPCs on 
micropatterned PA gels displayed only minor increases in size between 24 and $72 \mathrm{hrs}$ of culture (31\%) and showed no statistical differences in rate of activity (Figure 18F, 18G) demonstrating that maintaining small cell size preserved function despite the high stiffness of the substrate. Furthermore, when comparing the area vs rate of all of the conditions above, we noted that while small CPCs show a range of possible frequencies (between $0.5-3.5 \mathrm{~Hz}$ ), larger CPCs lost the capacity for higher rate activity (Figure $18 \mathrm{H})$. Collectively, these data indicate that maintenance of small cell size is beneficial for preserving the potential for high rate, rhythmic, activity in CPCs.

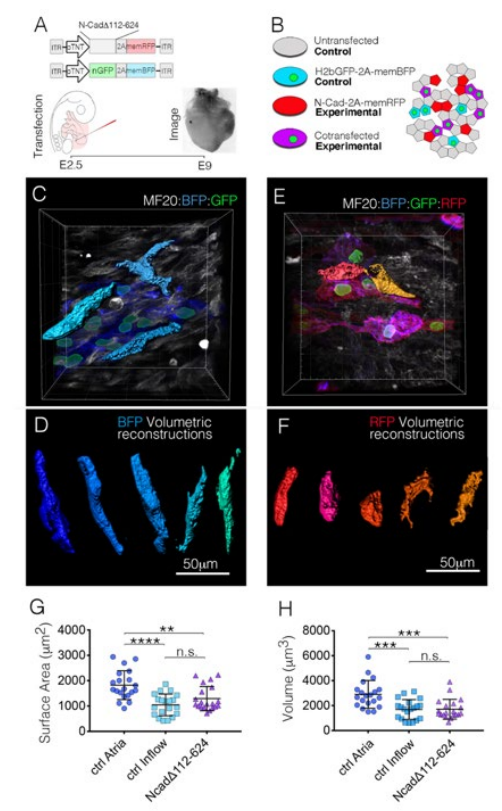

Figure 15. Inhibition of the cardiac AJ converts atrial WM to a CPC-like morphology.

A) Diagram of the experimental procedure for in vivo mosaic inhibition of AJ formation. B) Following manipulation, several cell populations are present in each heart. Control cells (nuclear GFP - Membrane BFP) were compared with experimental cells (nuclear GFP - Membrane BFP / N-Cad $\Delta 112-624$ - membrane RFP). C) in vivo imaging of control (nuclear GFP - Membrane BFP) atrial WM. Three of these cells were digitally isolated for volumetric reconstruction. D) Examples of in vivo volumetric reconstructions from control atrial WM. Scale Bar $-50 \mathrm{~mm}$ E) in vivo imaging of experimental (nuclear GFP - Membrane BFP/N-Cad $\Delta 112-624$ - membrane RFP) atrial WM. F) Typical examples of volumetric reconstructions from experimental cells. G) Quantification comparing surface area between control atrial WM $(n=20)$, control inflow CPCs $(n=20)$, and $N-C a d \Delta 112-624$ expressing atrial WM $(n=20)$. H) Quantification comparing cellular volume between control atrial WM $(n=20)$, control Inflow CPCs $(n=20)$, and N-Cad $\Delta 112-624$ expressing atrial WM $(n=20)$. Mean $+/-S D$ are indicated by line and bars for $\mathrm{G}$ and $\mathrm{H}$. 

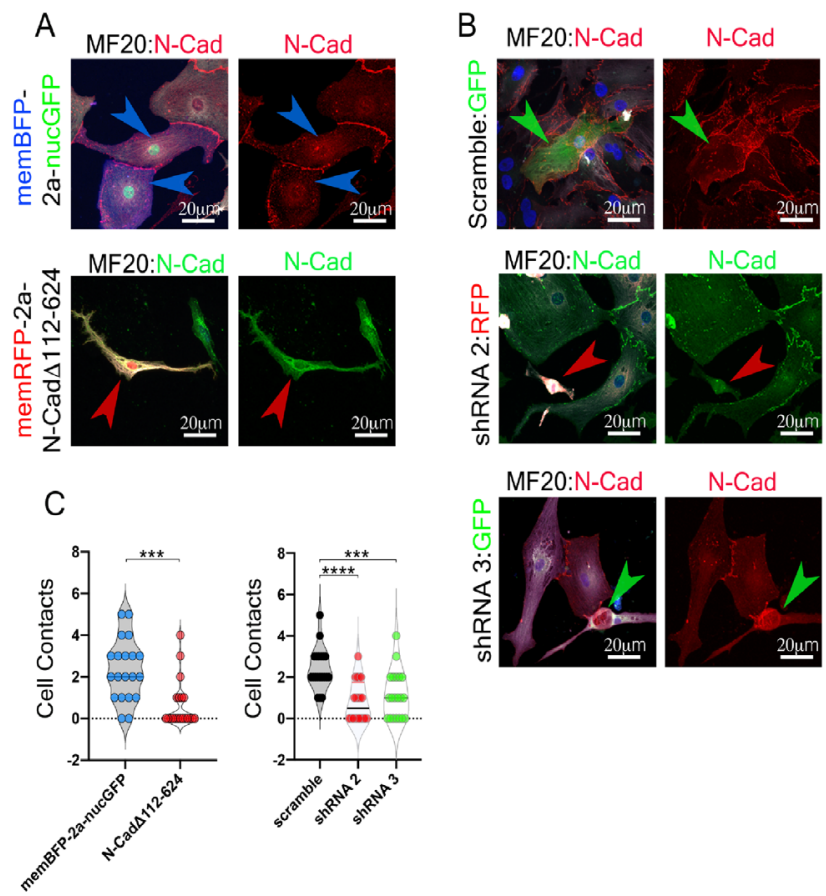

Figure 16. In vitro disruption of primary myocyte AJ formation.

A) Immunostaining for MF20 and N-cadherin in control cells expressing a membrane localized BFP and nuclear localized GFP (blue arrowheads indicate transfected cells) and cell expressing the D116-624 extracellular domain mutant of N-cadherin (red arrowheads). B) Immunostaining for Mf20 and N-cadherin in cells expressing either a scramble shRNA with a GFP reporter (green arrowheads) or shRNA's against Ncadherin. The N-Cad shRNA 2 plasmid contains an RFP reporter (red arrowheads indicate transfected cells), N-Cad shRNA3 contains a GFP reporter (Green arrowheads). For all conditions, cells were plated at a density of $1 \times 10^{5}$. Note that manipulated cells tended to isolate from adjacent myocytes. C) Quantification of the number of N-Cad positive cell contacts made among myocytes ( $n=20$ cells per group). Scale bars $-20 \mu m$.

\section{AJ formation regulates $\mathrm{CPC}$ gap junction formation}

In agreement with previous studies (Joyner and van Capelle 1986, Zhang, Holden et al. 2000, Glynn, Onal et al. 2014, Inada, Zhang et al. 2014), our computer modeling highlighted $g_{c}$ as an important feature for successful CPC function. Interestingly, several AJ-proteins are known to impact the stability of the gap junction (GJ) proteins that determine $g_{c}$ in the heart (Kostetskii, Li et al. 2005, Li 2005, Shaw, Fay et al. 2007, Smyth, Hong et al. 2010, Li, Goossens et al. 2012, Swope, Cheng et al. 2012). Therefore, we tested whether the regulation of AJ engagement might contribute to titration of CPC GJ coupling by examining AJ and GJ protein trafficking in primary embryonic CPCs. The GJ protein Connexin 40 (Cx40) was evaluated as our previous RNAseq data indicating it was the most abundant GJ transcript in 
all regions of the avian heart during early morphogenesis despite being more highly expressed in the WM versus CPCs (Bressan, Henley et al. 2018). CPCs and atrial WM were isolated and plated at low density and the distribution of $\mathrm{N}-\mathrm{Cad}$ and $\mathrm{Cx} 40$ was tracked. When either atrial WM or inflow cells were found in isolation (no cell-cell contacts), small puncta of $\mathrm{Cx} 40$ and $\mathrm{N}-\mathrm{Cad}$ were detectable throughout the cells (Figure 19A). However, in regions of the cultures where neighboring cells were in contact, intense N-Cad and $\mathrm{Cx} 40$ staining was detected along cell-cell interfaces (Figure 19A).

Upon examination of over 250 junctions, we noted that no $\mathrm{Cx} 40$ positive junction was detected independent from an AJ. To test whether the AJ directed GJ formation, we overexpressed the AJ blocking construct $(\mathrm{N}-\mathrm{cad} \Delta 112-624)$ in atrial $\mathrm{WM}$ and examined $\mathrm{Cx} 40$ localization in culture. Across 12 control cell pairs we measured $369.4 \square \mathrm{m}$ of total opposing cell membrane (distance between two cells of less than $2 \square \mathrm{m})$. Of this, $346 \square \mathrm{m}(93.7 \%)$ was composed of mixed N-cad/Cx40 positive junctions. Among Ncad $\Delta 112-624$ expressing cells we measured $270.8 \square \mathrm{m}$ of opposing membrane, of which only $65.4 \square \mathrm{m}$ (24.1\%) was composed of mixed junctions. Indeed, only 2 of $17 \mathrm{~N}$-cad $\Delta 112-624$ expressing atrial cells contained $\mathrm{Cx} 40$ positive junctions in contrast to 11 of 12 control cell pairs.

To test whether the AJ directly controlled Cx40 trafficking in CPCs, E6 atrial and inflow cells were isolated and cultured on cover glass coated with either FN or N-Cad. Following 72 hours of culture, cells were stained with vinculin, which localizes to both focal adhesions and AJs (Zemljic-Harpf, Manso et al. 2009), and Cx40 distribution was scored. Cx40 was rarely detected along a free edge or at the basal surface of atrial cells cultured on FN (Figure 20B). However, among atrial cells cultured on N-Cad, Cx40 dramatically re-localized toward the cover glass (Figure 20C). Similarly, inflow cells cultured on FN displayed large areas of vinculin staining along their basal surfaces, but these regions were largely devoid of Cx40 (Figures 19C,19D). Conversely, when cultured on N-Cad, both vinculin and Cx40 colocalized to the membrane in contact with the cover glass (Figures 19E,19F). To quantify this effect, we determined the proportion of $\mathrm{Cx} 40$ immunoreactivity proximal to the cover glass (basal $800 \mathrm{~nm}$ ) relative to the total volume of Cx40 immunoreactivity per cell. Independent of whether a cell originated from the inflow or 
atria, the presence of $\mathrm{N}$-Cad along the cells basal surface caused a significant redistribution of the total

$\mathrm{Cx} 40$ protein to the basal cell surface (Figures 19G,19H. Collectively, these data demonstrate that

disruption of the AJ blocks GJ formation and N-Cad engagement alone is sufficient to direct $\mathrm{Cx} 40$

trafficking to the cell surface in embryonic atrial WM and CPCs.
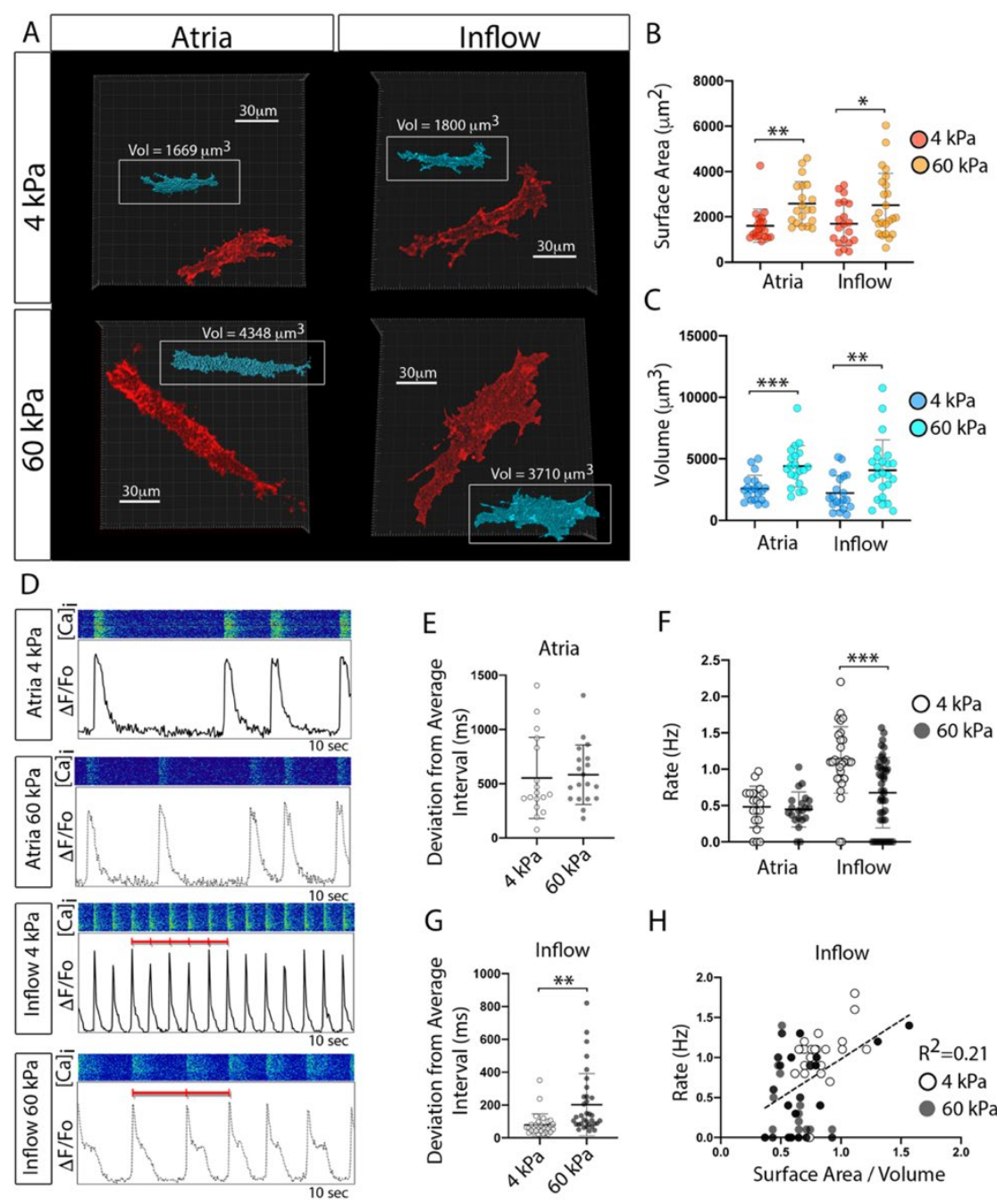

$\mathrm{H}$

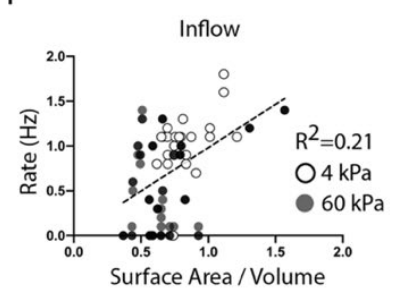

Figure 17. Force-mediated AJ engagement induces a WM-like phenotype in CPCs.

A) Maximum projection images of membrane RFP positive atrial WM and inflow cells cultured on $4 \mathrm{kPa}$ and $60 \mathrm{kPa}$ $\mathrm{N}$-Cad cross-linked, polyacrylamide gels. Insets (Blue) show volumetric reconstructs of each cell. Scale Bar - $30 \mathrm{~mm}$. B) Quantification of surface area of cells cultured as described for " $A$ " ( $n=40$ for atria, $n=44$ for inflow). C) Quantification of cellular volume for cells cultured as described in "A" D) Examples of typical physiological behavior of cells from "A-C." Intracellular calcium transients were recorded from cells cultured on different stiffnesses of NCad coated gels. E) Quantification of variation in transient interval on different gel stiffness for atrial cells $(\mathrm{n}=40)$. F) Quantification of rate of activation ( $\mathrm{n}=40$ for atria, $\mathrm{n}=44$ for inflow). G) Quantification of variation in transient interval on different gel stiffness for inflow cells $(\mathrm{n}=44)$. H) Linear regression between inflow cells surface area/volume ratio and rate of activity. Mean +/- SD are indicated by line and bars for B,C,E,F, and G. 
A
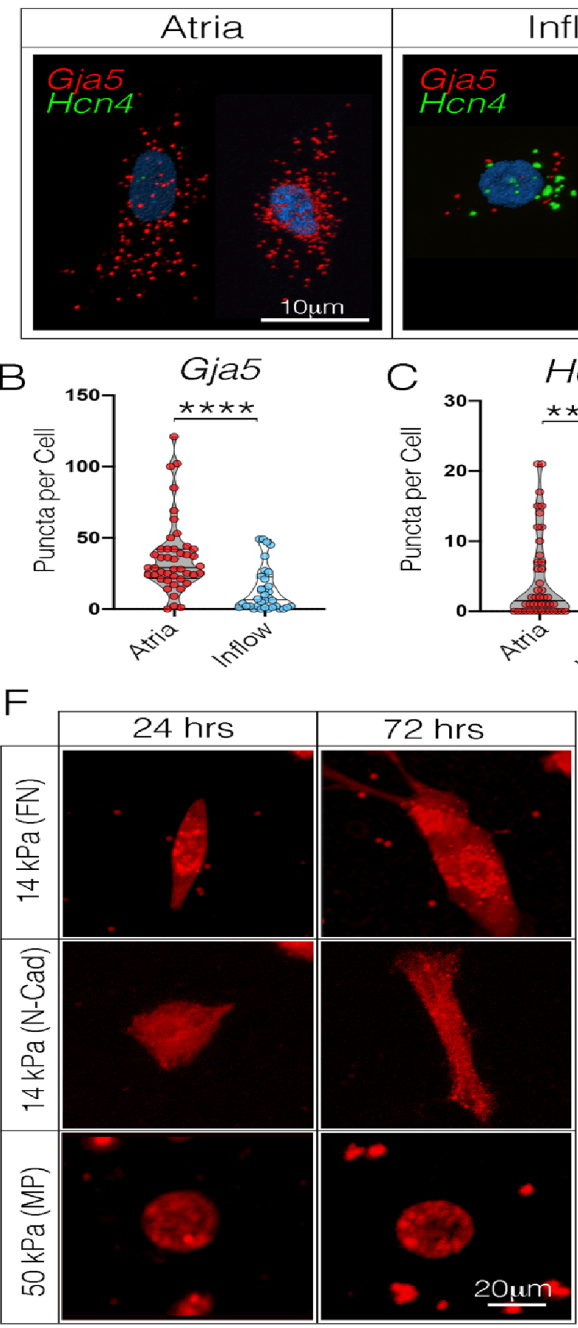
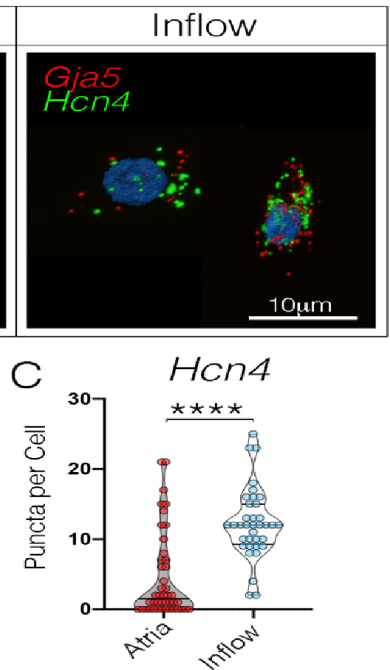

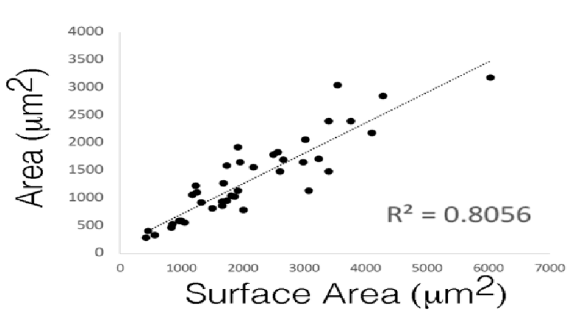

E

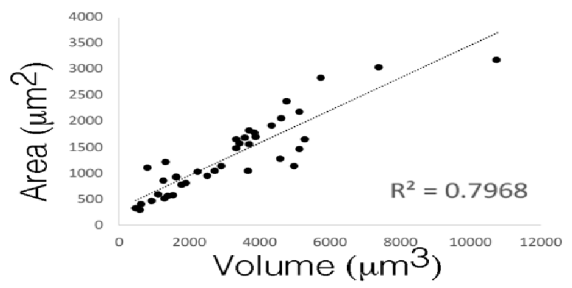

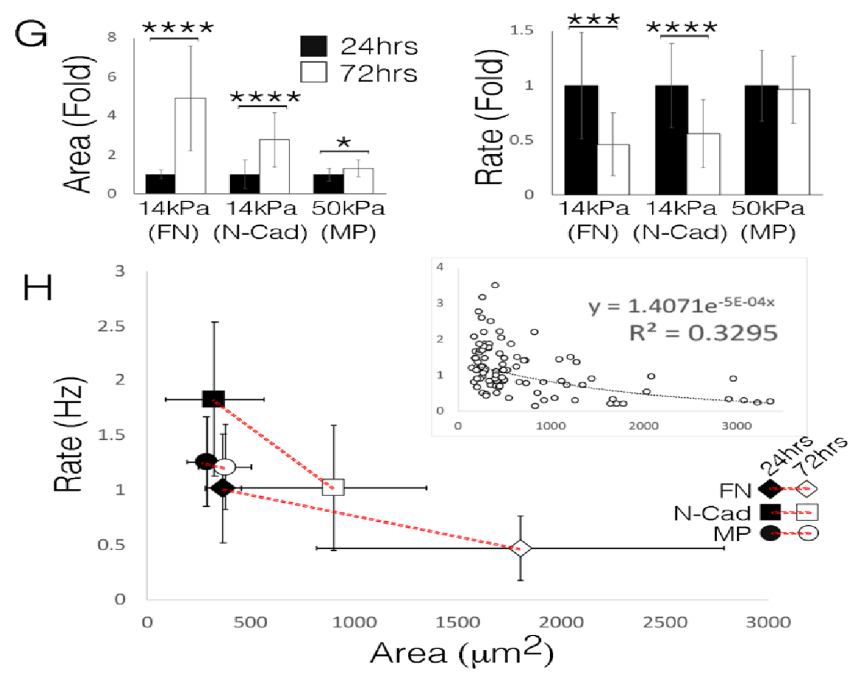

Figure 18. Increasing CPC size decrease capacity for high-rate electrical oscillation.

A) Validation of cell type following dissociation and isolation. RNAscope based fluorescent in situ hybridization was used to confirm molecular profiles $24 \mathrm{hrs}$. after isolation. Atrial WM express high levels of Gja5 (transcript encoding the gap junction protein $\mathrm{Cx} 40$ ) and inflow cells express high levels of the CPC

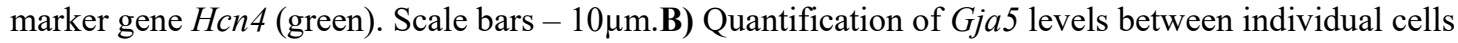
isolated from the atria and inflow. C) Quantification of Hcn4 levels between individual cells isolated from the atria and inflow. D) Correlation between $\mathrm{X}, \mathrm{Y}$ cell area and 3D surface area for the cells analyzed in Figure 8. E) Correlation between X,Y cell area volume for the cells analyzed in Figure 8. This was done to confirm that $\mathrm{X}, \mathrm{Y}$ area could be used as a proxy for cell size in experiments where internal size standards could not be used to normalize 3D imaging data (F-H below - see also STAR Methods). F) Membrane staining for Inflow cells cultured PA gels at 24 and $72 \mathrm{hr}$. time points. Cells were either plated on $14 \mathrm{kPa}$ Gels coated with fibronectin (FN) or N-Cad, or were plated on stiffer $50 \mathrm{kPa}$ micropatterned (MP) gels to constrain cell size over the culture period. Scale bar $-20 \mu \mathrm{m}$. G) Quantification of cell size and calcium transient rate for inflow cells plated on the gels from "F", (14 kPa FN n= 27; $14 \mathrm{kPa} \mathrm{N}-\mathrm{Cad} \mathrm{n}=40 ; 50 \mathrm{kPa}$ MP $n=28$ ). Mean +/- SD. H) Average rate vs average area for the conditions in " $F$ ". Change for each condition between 24 and $72 \mathrm{hrs}$. of cultures is noted by the red dashed lines. Inset shows all cells analyzed. Note: smaller cells have the capacity for rates $>1.5 \mathrm{hz}$ which is lost in cells greater than $\sim 1000 \mu \mathrm{m}^{2}$ 
$\underline{\mathrm{CPC} A J}$ engagement is regulated by regional enrichment of the transmembrane protein FLRT3

As described above, no statistical difference for N-Cad transcript levels were noted between WM and CPCs (Figures 14C,14D). We therefore focused on identifying factors that might post-

transcriptionally regulate AJ formation. We compared publicly available RNAseq data obtained from the SAN of E3 avian embryos(Bressan, Henley et al. 2018), E14.5 mouse embryos(Vedantham, Galang et al. 2015), and E17.5 mouse embryos(van Eif, Stefanovic et al. 2019) screening for genes that displayed at least two-fold enrichment over atrial WM in all three datasets (Figure 21A). This yielded a list of 53 commonly enriched genes. We then cross referenced this list with genes in the Gene Ontology (GO) term cell adhesion (GO:0007155), which further refined our candidate list to 12 factors. Fibronectin Leucine Rich Transmembrane Protein 3 (FLRT3) was identified among the common cell adhesion regulators through this approach. FLRT3 has previously been identified as a negative regulator of classical cadherin function (Ogata, Morokuma et al. 2007, Chen, Koh et al. 2009, Karaulanov, Böttcher et al. 2009) and as a factor capable of promoting homotypic cell sorting (Egea, Erlacher et al. 2008), but its function in heart development is not known(Maretto, Müller et al. 2008, Müller, Schulz et al. 2011). Therefore, we examined transcript and protein levels of FLRT3 during cardiogenesis. In agreement with the published RNAseq datasets, FLRT3 displayed highly specific enrichment in the inflow myocardium during stages when CPCs are structurally diverging from the WM (Figures 21B and 22B-22D). Importantly, FLRT3 protein was found to localize on the membrane of CPCs and was particularly enriched at cell contacts (Figures 21C and 21F). 

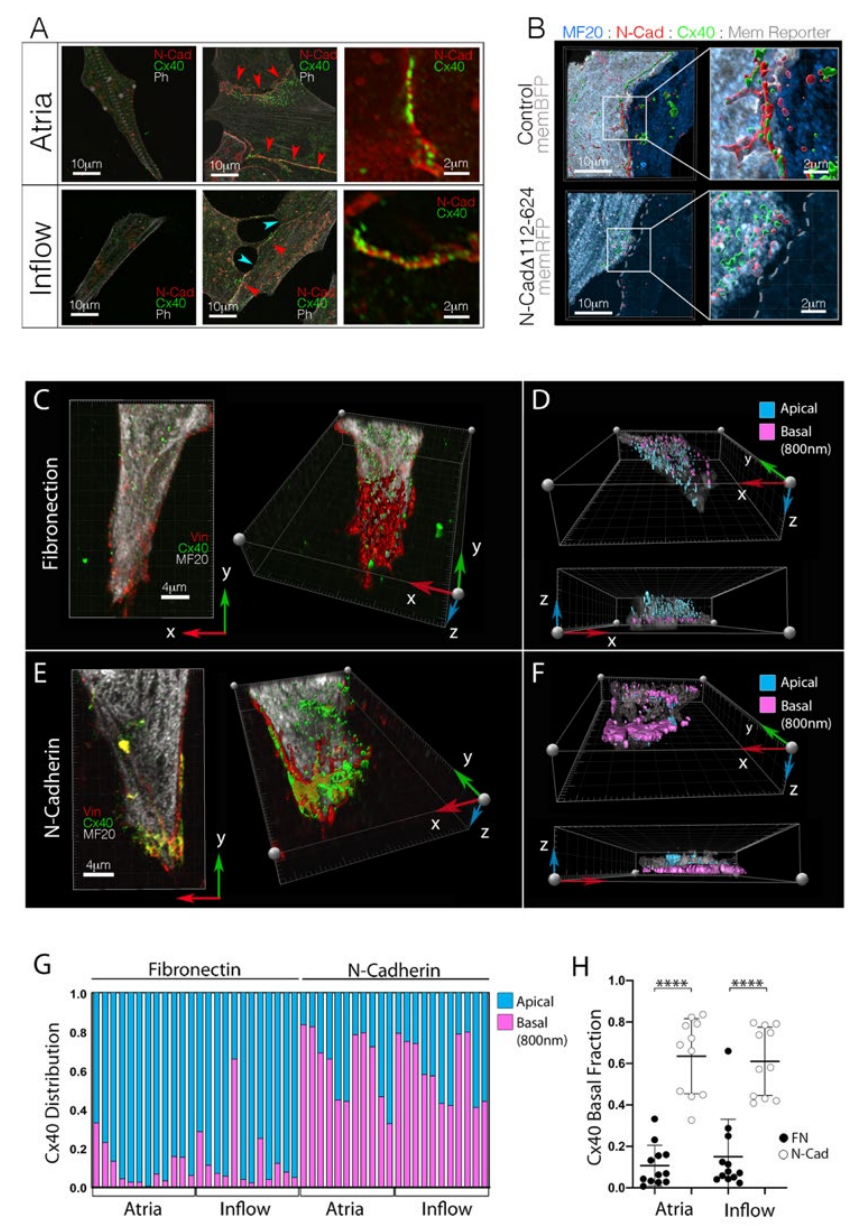

Figure 19. AJ and gap junction proteins colocalize within embryonic CPCs and atrial WM.

A) Cellular distribution of the AJ protein, N-Cad, and GJ protein, Cx40. Regardless of source tissue, isolated cells (no cell-cell contacts) show little N-Cad or Cx40 immunoreactivity. However, when cell junctions are present, both N$\mathrm{Cad}$ and $\mathrm{Cx} 40$ traffic to the cell-cell interface (red arrowheads). Note: inflow cells form junctions in culture, but they tend to show disrupted morphology (blue arrowheads). Scale bars - as indicated in panels. B) Representative images of N-Cad (red) and Cx40 (green) distribution in control vs N-Cad $\Delta 112-624$ overexpressing atrial WM cell pairs. Scale bars - as indicated in panels. C) Distribution of Vinculin (red) and Cx40 (green) in isolated inflow cell plated on Fibronectin. Cell is viewed from two angles: from above and a rotated view exposing the basal surface of the cell in contact with the fibronectin coating. Scale bars - 4mm D) Cell from "C," color coded to indicate distance of $\mathrm{Cx} 40$ positive staining from the basal surface of the cell. E) as in "C," for an inflow cell plated on a coverslip coated with N-Cadherin. F) As in "D," for the cell plated on N-Cad in "E." G) Quantification of Cx40 distribution in cells cultured either on Fibronectin or N-Cadherin. Each column represents an individual cell and the proportion of $\mathrm{Cx} 40$ immunoreactivity detected within 800nm of the coverslip is indicated in pink. H) Change in $\mathrm{Cx} 40$ distribution based on substrate composition. Mean +/- SD are indicated by line and bars. 

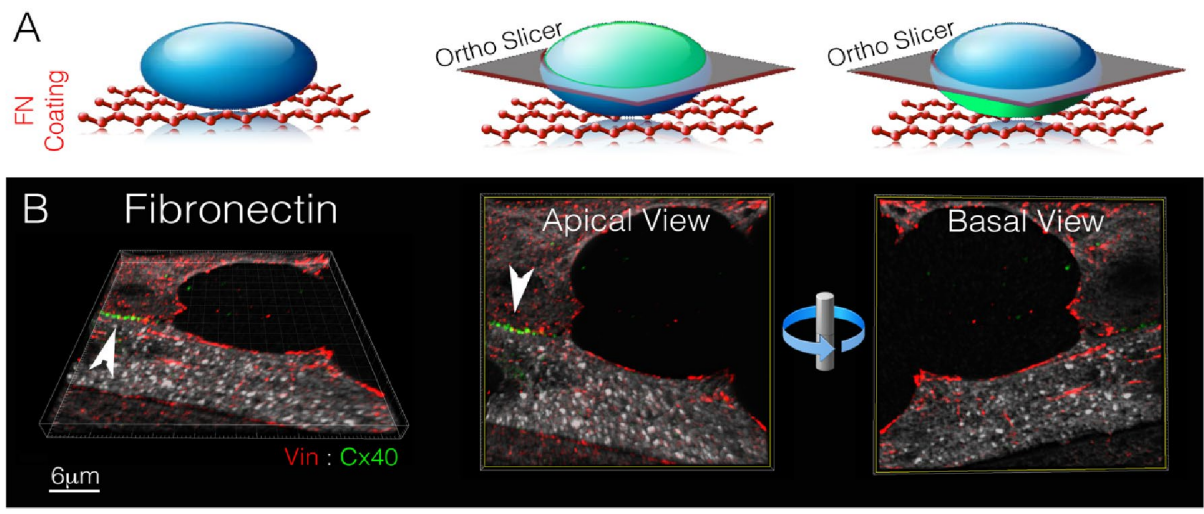

C
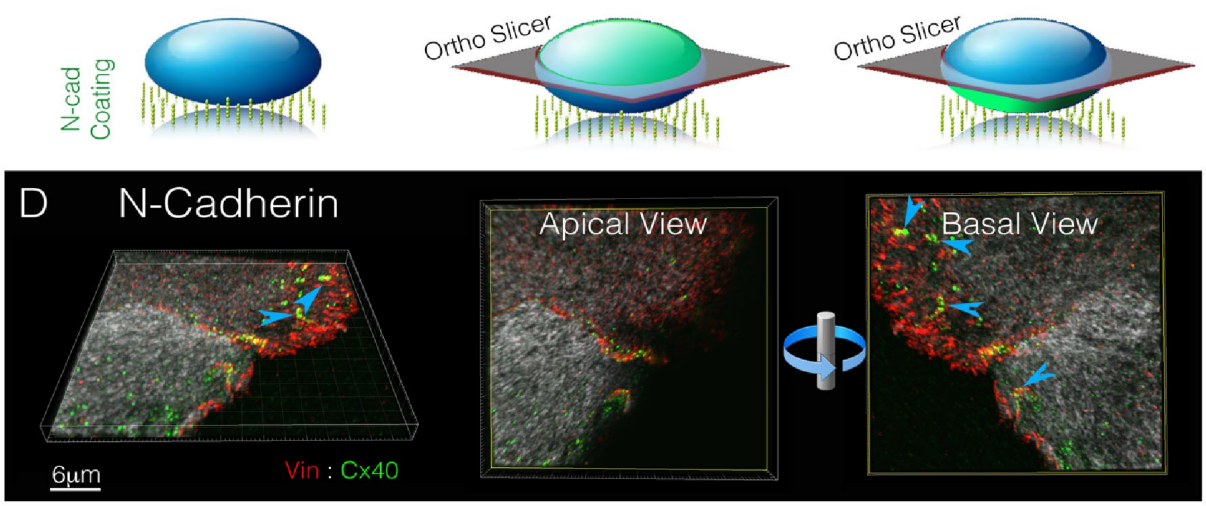

Figure 20. Cx40 re-localized to basal cell surface in atrial WM plated on $\mathrm{N}$-cad.

A) Diagram of dissociated atrial WM plated on cover glass coated with Fibronectin. Cells were stained with Vinculin (Vin -red) and Cx40 (green) and an orthogonal slicer was place 800nm up from the cell/cover glass interface to allow the apical and basal surfaces of the cell to be viewed independently. B) Image of typical atrial myocyte pair imaged as depicted in (A). Cx40 is localized to the cell-cell junction (White arrowhead). Scale bar $-6 \mu \mathrm{m}$. C) As in "A", diagram of atrial WM plated on N-Cadherin coated cover glass. D) Images of Atrial myocyte cultured on Ncad. Note that $\mathrm{Cx} 40$ broadly localized along the basal surface of the cell (blue arrowheads). Scale bar $-6 \mu \mathrm{m}$.

To test the function of FLRT3, we overexpressed FLRT3 in the WM outside the forming SAN (Figure 21D). Strikingly, mosaic overexpression of FLRT3 resulted in significant in vivo remodeling of myocardial tissue architecture (Figure 21E and 22G). FLRT3 overexpressing myocytes did not display the elongated aspect ratio present in most WM, but instead appeared small, rounded, and tightly clustered (Figure 21E). Due to the tight clustering of FLRT3 positive cells, it was difficult to determine the size of individual cells in vivo. Therefore, we dissociated FLTR3 overexpressing cells and plated them in dense, multi layered cultures $\left(1 \times 10^{6}\right.$ cells per $\left.\mathrm{cm}^{2}\right)$. In these cultures the majority of cells are not interacting with 
the substrate. Therefore, we imaged cells in the upper layers of the cultures and quantified cell area. Compared to control cells, FLRT3 overexpression resulted in a 2.3-fold decrease in area (Figure 22I). Additionally, we overexpressed an shRNA against FLRT3 in the SAN and atria (Tomás, Certal et al. 2011). While shFLRT3 did not affect atrial myocardial surface area or volume in vivo, it caused a statistically significant increases in surface area and volume in CPCs (Figures 22J, 22K).

The alteration in cellular morphology induced by FLRT3 lead us to examine whether it directly influenced cardiomyocyte AJ formation. To test this, hearts were transfected with control constructs, FLRT3 expression constructs, or an FLRT3 variant containing a two amino acid substitutions (FLRT3GG) predicted to disrupt the ability of this protein to influence classical cadherin localization (Ogata, Morokuma et al. 2007, Karaulanov, Böttcher et al. 2009). In control or FLRT3-GG expressing atrial cell pairs, long "ribbon-like" N-Cad positive junction were present (Figures 21F). However, N-Cad positive junctions were less frequent among FLRT3 expressing cells. Moreover, when junctions were detected, both N-Cad intensity and distribution around the junction were statistically diminished (Figure 21G-21K). Collectively, these data demonstrate that FLRT3 is highly enriched in the SAN, modulates the size of cardiomyocytes in a cell autonomous manner, and disrupts cardiac cell AJ engagement. 

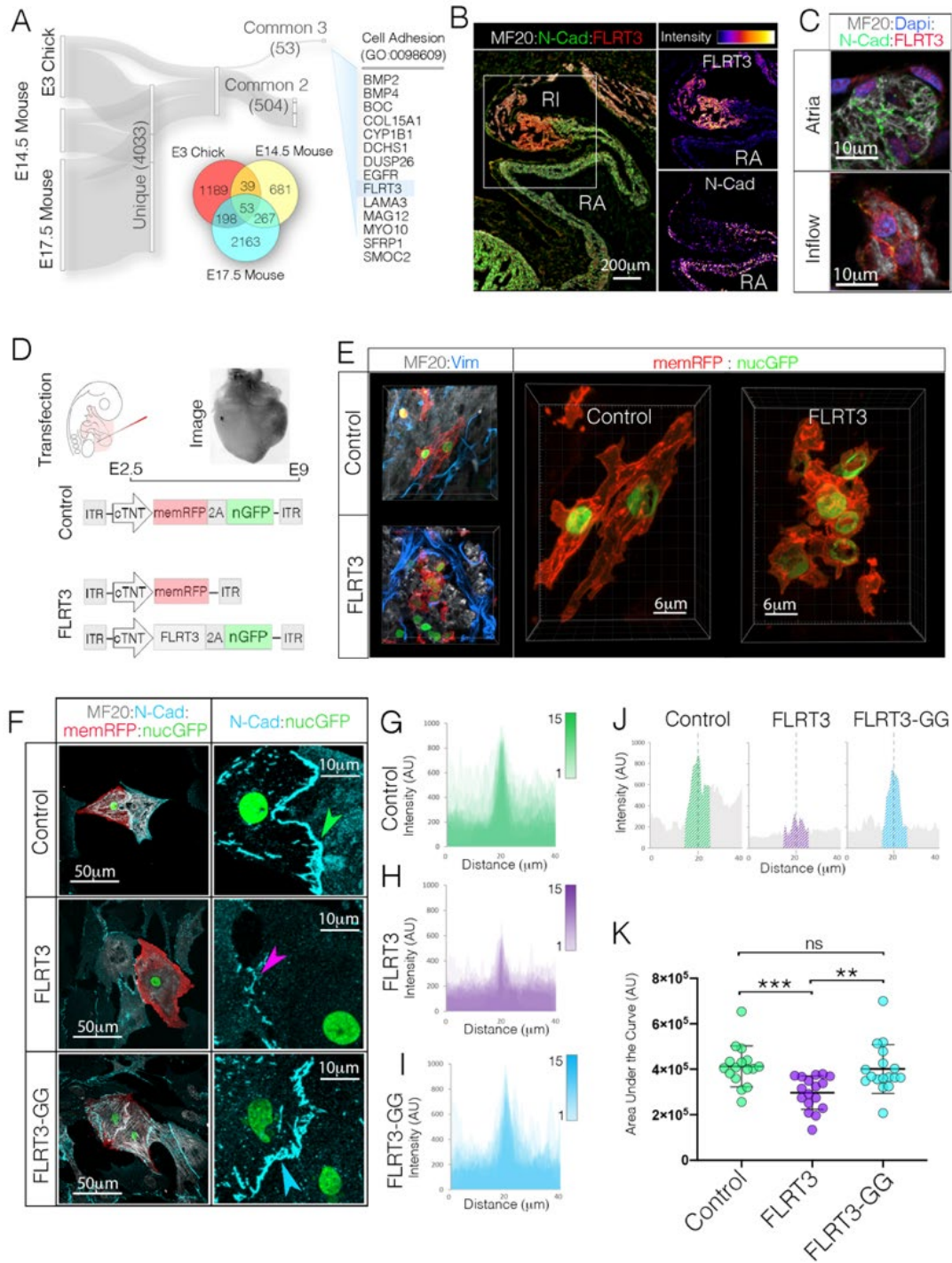

Figure 21. Negative Regulator of Cadherin Function, FLRT3, Disrupts CPC AJ Engagement.

A) RNAseq data sets from E3 Chick (GSE112894 (Bressan, Henley et al. 2018)), E14.5 Mouse (GSE65658 (Vedantham 2015)), and E17.5 Mouse (GSE125932 (van Eif, Stefanovic et al. 2019)) sinoatrial nodes were screened for common genes that displayed greater than 2-fold enrichment over atrial WM. The 53 genes common to all three data sets were then assessed for known biological functions related to cell adhesion. This analysis identified FLRT3 as a potential candidate regulator of CPC AJ formation. B) Immunohistochemical localization of FLRT3 and N-Cad. Single channel intensity plots demonstrate that the FLRT3 positive Inflow myocardium shows little immunoreactivity for N-Cad. Scale bar - 200mm C) Higher magnification imaging of FLRT3 and N-Cad immunostaining (see also Figures S7E, S7F). Scale bar-10mm. D) Diagram for FLRT3 in vivo over expression approach. E) Immunostaining for MF20 (white) and Vimentin (blue) in Control and FLRT3 overexpressing hearts. Scale bar - $6 \mathrm{~mm}$ F) Comparison of control, FLRT3, and FLRT3-GG expression on AJ formation in vitro. Scale bars - as indicated in panels. G) Overlay of intensity profiles of N-Cad staining across 15 control cell pairs. Position of cell-cell contact (ex. green arrowhead in "F") for each cell pair is superimposed at dotted line. H) As in "G," for cell pairs FLRT3 ( $\mathrm{n}=15)$. Position of cell-cell contact (ex. purple arrowhead in "F") are superimposed at dotted line. I) As in "G" and "H" for cells expressing FRLT3-GG $(\mathrm{n}=15)$. J) Intensity profile of a single N-cad junctions from control, FLRT3, and FLRT3-GG cells from "F". Hatched region indicates "area under the curve" quantified in "K." K) Quantification of N-Cad staining at cell contacts. Mean +/- SD are indicated by line and bars. 

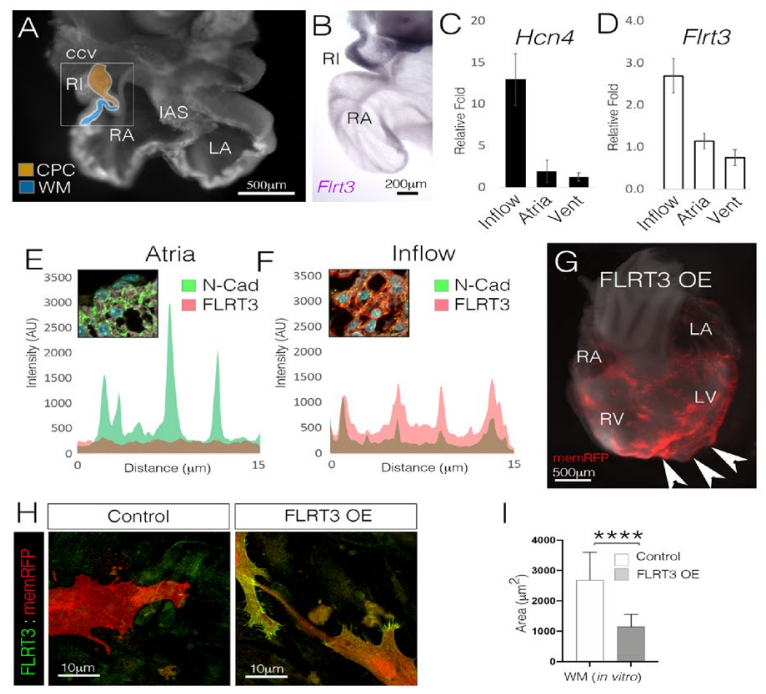

।
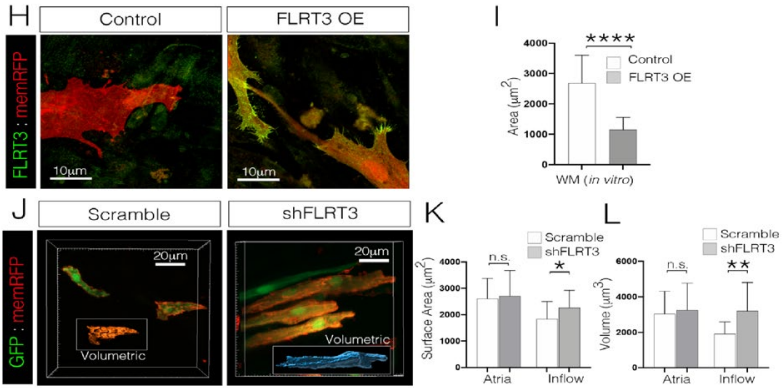

Figure 22. FLRT3 Localization and Overexpression.

A) Physiological definition of CPCs vs atrial WM in cardiac slice preparation from Figure 9. Scale bars $-500 \mu \mathrm{m} . \mathbf{B})$ Chromogenic RNA in situ hybridization on a cardiac slice. Note: FLRT3 expression (purple) is enriched within the $\mathrm{CPC}$ containing region defined in "A". Scale bars $-200 \mu \mathrm{m}$. C) Inflow myocardium was mechanically isolated an enrichment of the CPC marker, Hcn4, was confirmed by real-time PCR. Mean +/- SD. D) Real-time PCR was simultaneously used to confirm enrichment of Flrt3 transcript within the inflow compared to both atrial and ventricular myocardium. Mean +/- SD. E) Line scan of N-cad (green) and FLRT3 (red) immunostaining intensity from atrial WM (inset). F) Line scan of N-cad and FLRT3 immunostaining intensity within Inflow myocardium (inset). G) Typical morphology of heart following mosaic overexpression of FLRT3 in the WM. Note: truncation of the ventricular apex (white arrowheads). Scale bars $-500 \mu \mathrm{m}$. H) Confirmation of FLRT3 overexpression following transfection. Cells from transfected hearts were dissociated and FLRT3 protein expression/localization was validated using immunostaining. Scale bars $-10 \mu \mathrm{m}$. I) Quantification of cell surface area for Control vs FLRT3 overexpressing cells from "H" ( $\mathrm{n}=18$ control, 23 FLRT3 OE). Mean +/- SD. J) Inflow cells co-transfected either with a scramble or FLRT3 targeted shRNA (with a GFP reporter) and a membrane RFP reporter imaged in vivo. Insets show volumetric reconstructions of cells. Scale bars $-20 \mu \mathrm{m}$. K) Quantification of in vivo inflow and atria cell surface area following FLRT3 shRNA and scramble shRNA transfection. Mean +/- SD. L) Quantification of in vivo inflow and atria cell volume following FLRT3 shRNA and scramble shRNA transfection. Mean +/- SD.

\section{Discussion}

How the small number of CPCs found in the vertebrate heart are capable of maintaining their excitability while electrically interacting with large numbers of hyperpolarized WM has been a point of significant interest for the past 30 years(Joyner and van Capelle 1986). Several elegant models have emerged based on the architecture of the adult sinoatrial node (Joyner and van Capelle 1986, Zhang, 
Holden et al. 2000, Glynn, Onal et al. 2014, Inada, Zhang et al. 2014). The current paradigm holds that reduced coupling conductance serves to insulate CPCs (Joyner and van Capelle 1986, Fast and Kléber 1995, Rohr, Kucera et al. 1997, Shaw and Rudy 1997, Rohr, Kucera et al. 1998, Zhang, Holden et al. 2000, Kleber and Rudy 2004, Glynn, Onal et al. 2014, Inada, Zhang et al. 2014). The molecular mechanisms that impart the unique structure/function relationships in the hearts pacemaking complex, however, have yet to be determined. We examined this question under the assumption that aspects of CPC patterning that are required for their function should emerge during embryonic stages when ionic sourcesink relationships between CPCs and WM would become untenable to impulse generation. Indeed our mathematical modeling indicated that cell size and coupling would be predicted to influence CPC activity as cardiac morphogenesis proceeds, which was supported by our data that cells within the forming sinoatrial node remain relatively small throughout heart development and loss the ability to mechanically couple with one another via loss of AJs.

The AJ is a major mediator of mechanotransduction in a variety of cell types (Nagafuchi, Ishihara et al. 1994, Liu, Tan et al. 2010, Yonemura, Wada et al. 2010, Huveneers and de Rooij 2013, Barry, Tabdili et al. 2014). Furthermore, the cardiac AJ plays a direct role in maintaining the localization and stability of GJ proteins that electrically couple the WM in the adult heart (Kostetskii, Li et al. 2005, Li 2005, Shaw, Fay et al. 2007, Smyth, Hong et al. 2010, Li, Goossens et al. 2012, Swope, Cheng et al. 2012). As such, the lack of AJ formation among developing CPCs immediately led us to the hypothesis that disruption of cell junctions would serve to blunt the biomechanical inputs that stimulate hypertrophic growth in the WM, while simultaneously eliminating the interfaces required for stable GJ formation. Indeed, our data demonstrate that blocking AJ formation is sufficient to generate CPC-like morphological features outside of the forming SAN (Figure 15) and that presenting CPCs with a surface coated with NCad induces Cx40 trafficking to the plasma membrane (Figures 19E-19H).

Previous work has indicated that CPCs express transcription factors that suppress GJ expression (Hoogaars, Engel et al. 2007, Mommersteeg, Hoogaars et al. 2007, Espinoza-Lewis, Yu et al. 2009, 
Wiese, Grieskamp et al. 2009), which has led to a model in which transcriptional regulation of connexins determines CPC intracellular conductivity. Our data indicate that, in parallel to inhibition of mRNA production, embryonic CPCs also control extent of coupling by limiting connexin protein trafficking.

Somewhat surprisingly, both our simulations and cellular studies indicated that increasing CPC size negatively impacted function (Figures 9 and 17). How CPC size influences activity has remained unclear as previous reports have come to differing conclusions. Specifically, when the native heterogeneity of CPCs size present in the adult SAN has been compared to function, either no correlation was observed (Lyashkov, Juhaszova et al. 2007, Monfredi, Tsutsui et al. 2018), or larger cells isolated from the adult SAN were actually reported to have higher rates of activity (Honjo, Boyett et al. 1996). Conversely, increased CPC size due to aging is associated with decreased activity (Larson, Clair et al. 2013), and transient swelling of CPCs also lowers their firing rate(Lei and Kohl 1998). We see a dramatic impact on $\mathrm{CPC}$ function when these cells are placed under conditions that increase their size (Figures 17D, 17F-17G, and 18F-18H). Interestingly, among relatively small CPCs in our studies we do see a broad range of possible rates $(0.5-3.5 \mathrm{hz})$ which would agree with studies from adult CPCs (Lyashkov, Juhaszova et al. 2007, Monfredi, Tsutsui et al. 2018). However, CPCs that undergo induced hypertrophy lost the capacity for the higher rates of activity (Figures 11E-11G, 17D-17G, 18F-18H). Thus, from a developmental standpoint our data indicate that CPCs are capable of acquiring WM-like size characteristics and that events that prevent the hypertrophic growth that occurs in the WM may be necessary to preserve CPC function during cardiac morphogenesis. We would emphasize that our data do not implicate either the AJ or FLRT3 as regulators of cell fate decisions. Our data indicate that equal levels of N-Cad are present among CPCs and atrial WM at early developmental stages when CPCs have already functionally and molecularly diverged from the WM (Figures 11A and 13H-13I) (Bressan, Liu et al. 2013). As such, it appears that AJ engagement modulates characteristics such as cell size at time points well after lineage decisions have been made and serve as critical regulator of cellular features that impact CPC excitability. 
In summary, our current study highlights that during embryonic cardiac morphogenesis CPCs structurally diverge from the WM, attaining cytoarchitectural features that would be predicted advantageous for overall excitability. Through this process, CPCs are held back from undergoing the hypertrophic growth detected in the WM. Furthermore, CPCs uniquely lose AJ engagement during these stages, which mechanically isolates them while also lowering their capacity to electrically couple to neighboring cells. Final our data highlight the sinoatrial node enriched factor, FLRT3, as a cell autonomous modulator of myocyte $\mathrm{AJ}$ engagement. These findings indicate that regulation of $\mathrm{AJ}$ formation is a critical step in maintaining structure/function relationships during developmental CPC maturation and that $\mathrm{AJ}$ dynamics may need to be considered as rational design approaches for generating cellular-based "biological pacemakers" continue to evolve for clinical uses.

\section{Limitations of the Study}

We would highlight that increasing CPC size would not only be predicted to increase membrane capacitance but would also be predicted to affect the densities of the ionic currents required for electrical automaticity. In this report we do not address how individual channels change expression and/or localization in response to increasing cell size. As such our current data only indicate that increasing embryonic CPC size should negatively impact rate of activity and that the cells are not capable of acutely modulating current densities to compensate during the stages when they are structurally diverging from the WM. Similarly, we have used a culture system to induce hypertrophic growth in CPCs. We chose this system as it was not possible to examine functional differences among CPCs of differing sizes in vivo due to the syncytial electrical behavior of cardiomyocytes. As such, it is possible that mechanisms that allow CPCs to compensate for altered structure may exist in the developing heart and that such mechanisms are absent in our culture system. Finally, our data support a model by which electrical insulation can emerge as a product of decreased gap junctional density following AJ inhibition. Thus, mechanical insulation and electrical insulation would by intimately related. In the current study, however, we do not directly measure intercellular resistance between ether control or AJ manipulated CPCs. Therefore, we cannot rule 
out that an atypical form of electrical coupling that is below our resolution to detect can form independent of an AJ.

\section{Resource Availability}

\section{$\underline{\text { Lead contact }}$}

Further information and requests for resources and reagents should be directed to and will be fulfilled by the Lead Contact, Dr. Michael Bressan (Michael_Bressan@med.unc.edu).

Materials availability

All reagents generated in this study are available from the Lead Contact without restriction. $\underline{\text { Data and code availability }}$

The three-dimensional monodomain equations were used to simulate the conduction of the electrical propagation through embryonic cardiac tissue. The monodomain model can be derived from a homogenized description of the excitation propagation that neglects the specific details of the tissue microarchitecture(Keener and Sneyd 1998). In this model, the current density flux $\boldsymbol{J}$ is modeled using Ohm's law, such that $\boldsymbol{J}=-\boldsymbol{\sigma} \nabla V$, where $\boldsymbol{\sigma}$ is the tissue conductivity tensor and $V$ is the transmembrane potential difference. In general, the conductivity tensor $\boldsymbol{\sigma}$ includes information about the alignment of the cells, such that $\boldsymbol{\sigma}=\sigma^{t} \boldsymbol{I}+\left(\sigma^{l}-\sigma^{t}\right) \boldsymbol{f} \otimes \boldsymbol{f}$, in which $\boldsymbol{f}$ is a vector parallel to the average cellular orientation, $\boldsymbol{I}$ is the identity tensor, and $\sigma^{t}$ and $\sigma^{l}$ are the conductivity coefficients in the transverse and longitudinal cell directions, respectively. Because the transverse propagation is negligible in the simulations, we simplify the conductivity tensor to be isotropic, such that $\sigma=\sigma \boldsymbol{I}$, with $\sigma=\sigma^{l}=\sigma^{t}$. The monodomain model reads:

$$
\begin{aligned}
& \chi\left(C_{s p} \frac{\partial V}{\partial t}+I_{\text {ion }}(V, \boldsymbol{w})\right)=\nabla \cdot(\boldsymbol{\sigma} \nabla V), \\
& \frac{\partial \boldsymbol{w}}{\partial \mathrm{t}}=\mathrm{r}(\mathrm{V}, \boldsymbol{w})
\end{aligned}
$$


in which $\chi$ is the ratio of membrane surface to tissue volume, $C_{s p}$ is the specific membrane capacitance, $I_{\text {ion }}$ is the sum of the transmembrane currents per unit area which depend on the gating variables $\boldsymbol{w}$ and on the voltage $V$. In the following, $l$ and $w$ denote the length and width of the cell respectively, $\alpha$ the volume fraction of the extracellular space, $\sigma_{c}$ the cytosol conductance per unit length, and $g_{c}$ the gap junctional conductance. Following the approach of Hand et al.(Hand, Griffith et al. 2009), assuming a structured network of cells with uniform (averaged) electrical properties, we can link the macroscopic conductivity of the tissue to the cell dimensions via:

$$
\sigma=\sigma_{c}(1-\alpha)\left(1-\frac{1}{1+\kappa}\right), \quad \kappa=\frac{g_{c} l}{\sigma_{c} w^{2}}
$$

Assuming the membrane surface area $A$ and the cell capacitance $C$ are related by the equation $C_{m}$ $=C_{s p} A$, we can derive the cell dimensions $l$ and $w$ and the volume fraction of the extracellular space $\alpha$. More specifically, we assume 1:10 ratio between the $l$ and $w$, such that

$$
l=10 w, \quad w=2 \sqrt{\frac{A}{42 \pi}}, \quad \alpha=1-\frac{n_{C P C} V_{c}}{V_{C P C}}
$$

in which $V_{c}=\frac{\pi}{4} w^{2} l$ is the cell volume, $V_{C P C}$ is the volume of the region containing the CPCs and $n_{C P C}$ is the number of CPCs contained in $V_{C P C}$. Finally, the value of the membrane surface to tissue volume ratio $\chi$ is computed as $n_{C P C} A / V_{C P C}$.

We extract the values of $n_{C P C}$ and $V_{C P C}$ from imaging data. We derive the specific capacitance $C_{s p}$ in the CPCs region assuming CPCs are cylindrical and their capacitance changes with cell size between $20 \mathrm{pF}$ and $60 \mathrm{pF}$ as reported in the literature (Inada, Zhang et al. 2014). We use the modified Courtmanche-Ramirez-Nattel ionic model (Courtemanche, Ramirez et al. 1998, McDougal, Morse et al. 
2017) for the human atrial action potential to describe the ionic current density $I_{i o n}$ in the working myocardium. We use the CPC model of Fabbri et al. 2017 to represent the action potential in the SAN region. The monodomain model is solved using a finite linear element method based on piecewise linear finite elements along with a semi-implicit time-stepping scheme (Rossi and Griffith 2017).

Embryonic tissue geometry was segmented using Imaris (Bitplane). The three-dimensional geometry was prepared for simulations using MeshMixer (Autdodesk), QuadRemesher (Exoside) and Gmsh (Geuzaine and Remacle 2009). Simulations were run using the reconstructed tissue geometry and features including CPC slow diastolic depolarization, action potential duration, propagation pattern, and conduction velocities within and at the periphery of the forming sinoatrial node were compared back to recorded data from tissue slices. For sensitivity analysis $\left(C_{m}\right.$ vs $\left.g_{c}\right)$ slope of diastolic depolarization, action potential duration, and conduction velocities were allowed to diverge from recorded data and rate, rhythmicity, and the ability of the SAN to initiate an action potential and/or drive the atria was scored to determine parameters ranges that allowed for functional pacemaking (Figure 10).

The code developed in this work, BeatIt (available at github.com/rossisimone/beatit), relies on the parallel C++ finite element library libMesh (Kirk, Peterson et al. 2006) and on PETSc linear solvers (Balay, Gropp et al. 1997, Balay, Abhyankar et al. 2017). The visualization of the results and their analysis have been carried out using Paraview (Ahrens 2005) and MATLAB (The Mathworks, Inc.).

\section{Table 1: Values used for data and code analysis.}

\begin{tabular}{|c|c|c|c|c|c|c|c|c|}
\hline & $\begin{array}{l}C_{s p}[\mu \mathrm{F} / \\
\mathrm{cm}]\end{array}$ & $C_{m}[\mathrm{pF}]$ & $\begin{array}{r}\chi \\
{[1 / \mathrm{cm}]}\end{array}$ & $\begin{array}{l}\sigma \\
{[\mathrm{mS} / \mathrm{cm}]}\end{array}$ & $\begin{array}{c}\sigma_{c} \\
{[\mathrm{mS} / \mathrm{cm}]}\end{array}$ & $\begin{array}{r}g_{c} \\
{[\mathrm{nS}]}\end{array}$ & $\begin{array}{r}V_{C P C} \\
{\left[\mathrm{~cm}^{3}\right]}\end{array}$ & $n_{C P C}$ \\
\hline CPCs & 3 & $15-60$ & $1800-5640$ & $0.0045-0.1318$ & 6.7 & $0.3-3.3$ & $3.496 \mathrm{e}-6$ & 986 \\
\hline WM & 1 & - & 3400 & 1.0 & - & - & - & - \\
\hline
\end{tabular}




\section{Experimental Model and Subject Detail}

$\underline{\text { Animals }}$

Fertilized chicken eggs were obtained from Pilgrim's Farms (Sanford, NC, USA) and Allen Harim Hatchery (Liberty, NC, USA), and placed at $38^{\circ} \mathrm{C}$ in a humidified incubator. Eggs were incubated to desired developmental stages according to Hamburger-Hamilton(Hamburger and Hamilton 1992). Wildtype mice embryos were kindly provided by Joan Taylor. For immunofluorescence studies mice embryos were harvested at E13.5 for adherens junction protein expression analysis. Animal procedures and protocols were authorized by the University of North Carolina's American Association for Accreditation of Laboratory animal Care committee.

\section{Method Details}

$\underline{\text { Transfection }}$

Transfections were carried out as previously described (Goudy, Henley et al. 2019). In brief, chicken embryos were incubated at $38^{\circ} \mathrm{C}$ until Hamilton-Hamburger stage 16. Eggs were windowed and a DNA solution (Lipofectamine 3000 reagent (Invitrogen) mixed with plasmid DNA diluted in Opti-MEM (Gibco)) was micro-injected into the pericardial space surrounding the heart of the embryo. Eggs were sealed and placed in an incubator at $38^{\circ} \mathrm{C}$ until the desired developmental stage. Several plasmids were described and validated previously including: U6-NCad shRNA 1/2, ITR- $\alpha$ MHC-HyPBase-CAGpalmTagRFPII-2A-h2bEGFP-ITR, and ITR- $\alpha$ MHC-HyPBase-CAG-TagBFP-2A-h2bEGFP-ITR The ITR-aMHC-Hybase-CAG-palmTagBFP-2A-h2bEGFP-ITR was made by inserting palmTagBFP into the palmTagRFPII position of the palmTagRFPII-2A-h2bEGFP-ITR construct using Gibson Assembly cloning (GeneArt). The ITR- $\alpha$ MHC-Hybase-cNCad $\Delta 1$ 12-624-2A-palmTagRFPII-ITR construct was generated by subcloning a fragment of the chick N-cadherin cDNA lacking ectodomains 1-5 (Ong, Kim et al. 1998) into the ITR- $\alpha$ MHC-Hybase-CAG-palmTagRFPII-2A-h2bEGFP-ITR construct. The ITR-CMVHyBase-CAG-cFLRT3-2A-h2bEGFP-ITR and ITR-CMV-HyBase-CAG-cFLRT3[GG]-2A-h2bEGFPITR constructs were generated by cloning chick FLRT3 or a mutated chick FLRT3 in which amino acids 
579-580 were replaced with Glycines into the palmTagRFPII position of the previously described ITRCMV-HyBase-CAG-palmTagRFPII-2A-h2bEGFP-ITR (Goudy, Henley et al. 2019) using Gibson assembly (NEBuilder HiFi DNA Assembly Cloning Kit, New England Biolabs, Ipswich, MA). The ITRU6-FLRT3 shRNA-hPGK-EGFP-ITR plasmid containing the previously described shRNA AATTTCAGGCTACTGCTGCGATT (Tomas et al., 2011), was synthesized by VectorBuilder Inc. (Chicago, IL).

Optical Mapping and Calcium Imaging

Optical mapping (voltage Imaging) analysis was done using previously described methods (Bressan, Liu et al. 2013, Bressan, Yang et al. 2014, Bressan, Louie et al. 2014, Bressan, Henley et al. 2018). Briefly, inflow and atrial tissue were explanted and placed at $37^{\circ} \mathrm{C}$ in fresh $1 \mathrm{X}$ HBSS $(1.26 \mathrm{mM}$ $\mathrm{CaCl} 2$ anhydrous, $0.49 \mathrm{mM} \mathrm{MgCl2}-6 \mathrm{H} 2 \mathrm{O}, 0.41 \mathrm{mM} \mathrm{MgSO} 4-7 \mathrm{H} 2 \mathrm{O}, 5.33 \mathrm{mM} \mathrm{KCl}, 0.44 \mathrm{mM} \mathrm{KH} 2 \mathrm{PO}$, 4.17 mM NaHCO3, 137.93 NaCl, 0.33 mM Na2HPO4 anhydrous, 5.56 mM D-Glucose) solution for 10 minutes to recover. Explants were then place into a staining solution of $10 \mu \mathrm{M}$ Di-4 ANEPPS (Invitrogen) voltage sensitive dye and $10 \mu \mathrm{M}(-)$ Blebbistatin (Millipore Sigma) in $1 \mathrm{X}$ HBSS for 10 minutes at $37^{\circ} \mathrm{C}$ with 95\% O2, and 5\% CO2 incubation. After incubation, explants' action potentials were recorded in $1 \mathrm{X}$ HBSS solution perfused with $95 \% \mathrm{O} 2$, and $5 \% \mathrm{CO} 2$ on a temperature-controlled imaging chamber $(36+/-$ $\left.0.5^{\circ} \mathrm{C}\right)$

Calcium transient activity of cultured cells was imaged by staining cells with Cal-520, AM (AAT Bioquest, Sunnyvale, CA); $5 \mathrm{uM}$ in in DMEM/F12 containing 15\% FBS. Cultures were allowed to recover for 30 minutes at $37^{\circ} \mathrm{C}$ with $5 \% \mathrm{CO}$. Following recovery individual cells were imaged for calcium activity in a Tokai Hit Stage Top Incubator at $37^{\circ} \mathrm{C}$ and $5 \% \mathrm{CO}$. Analysis and quantification methods are described below.

Cytopainter - Cell Plasma Membrane Staining Kit - Orange Fluorescence (Abcam, Cambridge, MA) was used for a subset of cells that did not contain a genetic membrane (Figures 18F-18K). Prior to plating on micropatterned polyacrylamide gels, dissociated cell pellets were stained with assay buffer 
containing $1 \mathrm{X}$ staining solution. The pellets were incubated at $37^{\circ} \mathrm{C}$ with $5 \% \mathrm{CO} 2$ for 20 minutes. After 3-5 washes in complete media (15\% FBS in DMEM), cells were resuspended and plated on PA gels. Micro-electrode Array Recordings

At the desired developmental stage, heart tissue was isolated using surgical forceps, clipper neuro scissors (Fine Science Tools), and curved tenotomy scissors and placed in pre-warmed 1X HBSS solution. Explants in $1 \mathrm{X}$ HBSS were then placed at $37^{\circ} \mathrm{C}$ for 25 minutes to recover. Explants were then transferred to a 6-well MEA recording chamber (Multichannel systems) precoated with fibronectin (Corning) at $2 \mu \mathrm{g} / \mathrm{cm}$ and pre-warmed to $37^{\circ} \mathrm{C}$. Explants were cultured in Dulbecco's Modified Eagle's Medium (Sigma Aldrich), 15\% fetal bovine serum (Gibco), and 1\% Penicillin-Streptomycin (Gibco) for $16 \mathrm{hrs}$ to allow for adherence to the MEA. Three sets of field potential recordings were taken per explant (60 seconds per recording) using three technical replicates per group. Data were recorded and analyzed using MC_Rack V.4.6.2 and MEA_select V.1.3.0 software (Multichannel systems).

$\underline{\text { Immunofluorescence }}$

Whole mount - Whole hearts were fixed in $4 \%$ PFA at $4^{\circ} \mathrm{C}$, rocking for 1 hour, then washed three times with 1X DPBS (2.67 mM KCl, 1.47 mM KH2PO4, 137.93 mM NaCl, 8.06 mM Na2HPO4-7H2O) with $0.1 \%$ Tween-20 for 20 minutes. Tissue was blocked at room temperature in 1x DPBS, $1 \%$ BSA, 0.1\% Tween-20 for 2 hours. Simultaneously, the primary antibody MF20 (Invitrogen), was blocked at 1:500. Primary antibody was applied at $4^{\circ} \mathrm{C}$ overnight followed by six 30 minute washes at room temperature in $1 \mathrm{X}$ DPBS. Secondary antibody (1:500) and DAPI (1:2000) were applied overnight at $4^{\circ} \mathrm{C}$ in $1 \mathrm{X}$ DPBT. The following day tissue was washed six times at room temperature in $1 \mathrm{X}$ DPBS for 30 minutes in the dark.

Thick section -Whole hearts were fixed in $4 \%$ PFA at $4{ }^{\circ} \mathrm{C}$, rocking for 20 minutes, then washed three times with $1 \mathrm{X}$ DPBS with $0.1 \%$ Tween-20 (1X DPBT) for 15 minutes. Tissue was blocked at room temperature in $1 \mathrm{X}$ DPBS, $1 \%$ BSA, $0.1 \%$ Tween-20 for 1 hour. Simultaneously, the primary antibodies MF20 (Invitrogen) and N-Cadherin (Millipore Sigma), were blocked at 1:500 and 1:500 dilutions, 
respectively. Primary antibodies were applied at $4{ }^{\circ} \mathrm{C}$ overnight followed by four 15 minute washes at room temperature in 1 X DPBS. Secondary antibodies (1:500) were applied at room temperature in the dark for 2 hours in 1X DPBS. Tissue was washed 4 times with 1X DPBT for 15 minutes followed by application of DAPI (1:1000) for 30 minutes at room temperature in the dark. Tissues were washed three times with 1 X DPBT for 15 minutes at room temperature in the dark.

Thin section - Paraffin sections and cryosections were prepared using standard techniques.

Paraffin sections were heated to $55^{\circ} \mathrm{C}$ for 15 minutes and were deparaffinized using xylene washes for 60 minutes. Sections were then rehydrated through a graded ethanol series. Antigen retrieval was performed for 30 minutes in citrate buffer (10 mM Tri-sodium citrate, $0.05 \%$ Tween-20, $\mathrm{pH} 6.0$ with HCl). Slides were allowed to cool and then washed with $1 \mathrm{X}$ DPBS for 5 minutes and blocked (1X DPBS, 1\% BSA, 0.1\% Tween-20) for 1 hour concurrent with antibody blocking. Primary antibodies MF20, N-cadherin, catenin (ThermoFisher Scientific), and $\alpha \mathrm{T}$-catenin (CTNNA - Abcam) were blocked at 1:500 dilution. Slides were incubated at $4^{\circ} \mathrm{C}$ overnight in primary antibody. Slides were washed three times with $1 \mathrm{X}$ DPBS for 10 minutes. Secondary antibodies were applied at 1:500 dilutions in 1X DPBS for 1 hour in the dark and were washed three times in 1X DPBS for 10 minutes. Cryosections were washed three times with $1 \mathrm{X}$ DPBS with $0.1 \%$ Tween-20 (1X DPBT) for 5 minutes and blocked (1X DPBS, 1\% BSA, 0.1\% Tween-20) for 1 hour concurrent with antibody blocking. Primary antibodies, MF20, N-cadherin, catenin, and $\alpha \mathrm{T}$-catenin, were blocked at 1:500 dilution. Slides were incubated at $4^{\circ} \mathrm{C}$ overnight in primary antibody. Slides were washed three times with 1X DPBS for 10 minutes. Secondary antibodies were applied at 1:500 dilutions in 1 X DPBS for 1 hour in the dark and washed off using three 10 minutes washes in $1 \mathrm{X}$ DPBS. Slides were mounted with a 24x55 microscope cover glass (Fisher Scientific) and Flouroshield mounting media with DAPI (Abcam) or ProLong Gold (Invitrogen).

\section{$\underline{\text { Cell Dissociation }}$}

Dissociations were carried out using previously described protocols (Goudy, Henley et al. 2019). Transfected isolated cardiac tissue was placed in a $0.17 \%$ trypsin (Sigma-Aldrich, St. Lois, MO) solution 
for 30 minutes and centrifuged at $3000 \mathrm{rpm}$ for 5 minutes to obtain a pellet of cells. After 3-5 washes in complete media (15\% FBS in DMEM), cells were plated on human fibronectin coated 8-well culture slides (Corning) or 35/10 mm glass bottom culture dishes (Greinerbio one). Cultures were fixed at $4^{\circ} \mathrm{C}$ for 30 minutes in 4\% PFA. Immunohistochemistry was carried out using N-cadherin, MF20, Connexin-40 (Life Span Life Sciences), GFP (Invitrogen), and Alexa Fluor 647 Phalloidin (Invitrogen) primary antibodies.

\section{$\underline{\text { RNAscope In Situ Hybridization }}$}

Fluorescent RNA staining was performed using the RNAScope Multiplex Fluorescent Assay (ACDBio, Newark, CA) following manufacturer's protocol for cultured adherent cells. Cultures were fixed in $4 \%$ PFA at $4^{\circ} \mathrm{C}$ for 30 minutes, permeabilized by washing three times with $1 \mathrm{X}$ DPBT for 10 minutes and dehydrated in a graded ethanol series at $4{ }^{\circ} \mathrm{C}$. Cells were then rehydrated at $4^{\circ} \mathrm{C}$ and treated with Protease III (1:150 in 1X DPBT) for 10 minutes at room temperature and washed with 1X DPBT. Hybridization with the target probes $\left(\mathrm{Hcn} 4, \mathrm{Gja} 5\right.$, Flrt3) was carried out at $40^{\circ} \mathrm{C}$ for 2 hours. Cultures were then immersed in an alternating series of wash buffers at room temperature and fluorescent amplification buffers at $40^{\circ} \mathrm{C}$ before being counterstained with DAPI and mounted with ProLong Gold (Invitrogen). Cultures were stored at $4^{\circ} \mathrm{C}$ until imaging.

\section{$\underline{\text { Polyacrylamide gels }}$}

Polyacrylamide (PA) gels were prepared using previously described methods(Chopra, Tabdanov et al. 2011). Commercially available solutions of $40 \%$ acrylamide and $2 \%$ bisacrylamide (Sigma-Aldrich, St. Louis, MO) were added to final concentrations of $7.5 \%$ and $0.05 \%$ respectively or $10 \%$ and $0.5 \%$ respectively to produce gels of $4 \mathrm{kPa}$ and $60 \mathrm{kPa}$ sheer moduli. Beads of $2 \mu \mathrm{m}$ diameter (Thermo Fisher Scientific, Waltham, MA) were added to the acrylamide solutions as 3D volumetric standards for downstream analysis. To polymerize, $2 \mu \mathrm{L}$ tetramethylene diamine (Sigma-Aldrich, St. Louis, MO) and $200 \mu \mathrm{L}$ of $1 \%$ ammonium persulfate (Sigma-Aldrich, St. Louis, MO) were added to the solutions to a total volume of $2 \mathrm{~mL}$ in $1 \mathrm{X}$ DPBS. A $400 \mu \mathrm{L}$ aliquot of the polyacrylamide was added to a $35 / 10 \mathrm{~mm}$ 
glass bottom dish (Greiner Bio One, Monroe, NC). Dishes were plasma activated (Harrick Plasma, Ithaca, NY) before being treated with 3-aminopropyltriethanolamine (Sigma-Aldrich, St. Louis, MO) and glutaraldehyde (Sigma-Aldrich, St. Louis, MO). Gels were covered with a 1 inch diameter by $1 / 16$ inch thick quartz coverslip (Thermo Fisher Scientific, Waltham, MA) pretreated with Sigmacote (SigmaAldrich, St. Louis, MO). After a 30-minute polymerization step, the coverslip was removed and a solution of $50 \mathrm{mM}$ HEPES buffer ( $\mathrm{pH}$ 8) containing the cross-linker sulfo-N-sulfosuccinimidyl-6-(4'-azido-2'nitrophenylamino) hexanoate (Sigma-Aldrich, St. Louis, MO) at a concentration of $0.5 \mathrm{mg} / \mathrm{mL}$ was added to the dishes, submerging the gels. The dishes were then placed under ultraviolet light (Jetlight Company, Inc., Irvine, California) and irradiated for 7 minutes. Anti-Human Fc antibody (Thermo Fisher Scientific, Waltham, MA) was added at a concentration of $30 \mu \mathrm{g} / \mathrm{cm}^{2}$ and incubated overnight at $4{ }^{\circ} \mathrm{C}$ with rocking. After washes in 50 mM HEPES buffer, recombinant Human N-cadherin Fc chimera (R\&D Systems, Minneapolis, MN) was added at a concentration of $10 \mu \mathrm{g} / \mathrm{cm}^{2}$ and incubated overnight at room temperature with rocking. PA gels were then incubated with 1.5\% BSA in $50 \mathrm{mM}$ HEPES at room temperature for 1 hour and washed prior to cell plating. Micropatterned gels were custom fabricated from $50 \mathrm{kPa}$ PA (4Dcell). Single cells seeded on 20um diameter micropatterns were selected for imaging.

\section{Transmission Electron Microscopy (TEM)}

E6 chicken embryos were fixed overnight in $4 \%$ PFA at $4{ }^{\circ} \mathrm{C}$ and washed three times in $1 \mathrm{X}$ DPBS for 20 minutes. Embryos were embedded in 3\% low melting temperature agarose (LMA, Apex) in 1X DPBS. Embedded tissue was cut on a Vibratome into $200 \mu \mathrm{m}$ thick sections. Sections were immersionfixed in 4\% paraformaldehyde/1\% glutaraldehyde/0.1M sodium phosphate, $\mathrm{pH} 7.4$, and stored for several days at $4^{\circ} \mathrm{C}$. After post-fixation for 1 hour in potassium ferrocyanide-reduced osmium $(1 \%$ osmium tetroxide/1.25\% potassium ferrocyanide/0.1M sodium phosphate buffer), samples were dehydrated through a graded series of ethanol $(30 \%, 50 \%, 75 \%, 90 \%, 100 \%, 100 \%)$ and propylene oxide, then infiltrated and embedded in PolyBed 812 epoxy resin (Polysciences, Warrington, PA). Using a diamond knife, $1 \mu \mathrm{m}$ semi-thin sections were cut, mounted on slides, and stained with $1 \%$ toluidine blue to 
examine by light microscopy and isolate the region of interest. Ultrathin sections $(70-80 \mathrm{~nm})$ were cut with a diamond knife and mounted on 200 mesh copper grids followed by staining with $4 \%$ aqueous uranyl acetate for 12 minutes and lead citrate for 8 minutes.

Stained sections were imaged with a ThermoFisher (FEI) Tecnai 12 G2 transmission electron microscope (Hillsboro, OR) operated at $80 \mathrm{kV}$ using a 1k x 1k CCD camera (Model 794, Gatan, Pleasanton, CA) equipped with Digital Montage software or at $120 \mathrm{kV}$ using a $4 \mathrm{k}$ x $4 \mathrm{k}$ CMOS camera (Model Rio16, Gatan, Pleasanton, CA). CPCs were defined as the cell population located in the right inflow myocardium overlapping with the action potential initiation site (Figure 9A) (Bressan, Liu et al. 2013, Bressan, Henley et al. 2018). This region was also confirmed to be enriched for Hcn4, Shox2, Bmp2, Smoc2, and depressed in Nkx2.5 and Gja5 expression as previously reported (Christoffels, Mommersteeg et al. 2006, Espinoza-Lewis, Yu et al. 2009, Ye, Wang et al. 2015, van Eif, Stefanovic et al. 2019).

$\underline{\text { RT-qPCR }}$

Cardiac tissue was isolated and placed in TRIzol (Invitrogen) and RNA was extracted using manufacturer's protocol for PureLink RNA Mini Kit (Invitrogen). Approximately 20 ng of RNA was obtained from each sample and cDNA was generated using Superscript IV according to the manufacturer's guidelines (Invitrogen). RT-qPCR was performed on a Quantastudio 6 Flex Real-Time PCR System (Invitrogen) using iTaq 2X SYBR Green Supermix (Bio-Rad) with 3 biological replicates, run in triplicate. HCN4 (Forward primer: GCGACTTCAGGTTTTATTGGG Reverse primer: GAGGACAAGGTCGATGAGGA), N-cadherin (Forward primer: ATGGCAAATGAAGGTGAAGC Reverse primer: CTTCAGATGGCTGCTGTCCT), $\alpha \mathrm{T}$-catenin (Forward primer: TTTGTGCGGTATATTGCTCAGAAG Reverse primer: CTTGGAGATACTGGCTTTAAGATGC), FLRT3 (Forward Primer: GAACAGCCACAGTGAAAGCA Reverse Primer: TCGATGCATTCAGGTGTCTC) and gamma-tubulin (Forward primer: CAACTTCGATGAGCTGGACA Reverse primer: CCTCTTGTCCCCATGTCACT), were amplified and 
expression levels were normalized to Gapdh (Forward primer: CCCCCAATGTCTCTGTTGTT Reverse primer: CATCCAAGGTGGAGGAATGG). Calculated fold changes are relative to the atria.

\section{Quantification and Statistical Analysis}

$\underline{\text { Microscopy }}$

Optical mapping samples were recorded using a CMOS Camera (MiCAM Ultima, SciMedia) and vertical THT Microscope (SciMedia). The data was processed using BV-Ana software (SciMedia). TEM samples were observed using a JEOL JEM-1230 transmission electron microscope operating at $80 \mathrm{kV}$ (JEOL USA, Inc., Peabody, MA) and images were acquired with a Gatan Orius SC1000 CCD Digital Camera and Gatan Microscopy Suite 3.0 software (Gatan, Inc., Pleasanton, CA). Whole mount and thick section images were acquired using the LSM 800 confocal microscope (Zeiss) and 25x liquid immersion lens (NA 1.4). Thin sections were imaged using the Olympus IX-81 widefield inverted microscope and Metamorph software. Dissociated cell cultures were imaged on a LSM 880 laser scanning confocal microscope with an Airyscan super resolution sensor. Post processing was performed using ZEN Blue Imaging software (Zeiss). Cells on polyacrylamide gels were imaged using an Olympus FV3000RS confocal microscope with a live cell Tokai Hit stage-top incubator. 3D reconstruction was carried out by batch deconvolution of confocal images in AutoQuantX3 (Media Cybernetics). The deconvolution files were then opened in IMARIS 9.3 software (Bitplane) to render individual cell surfaces for quantification of cell features such as surface area and volume. Calcium transient data was processed using ImageJ V2.0.0 (NIH). RNAScope data was analyzed using ImageJ V2.0.0 (NIH). All statistical analysis of data was assessed using a two-tailed unpaired Student t-test in Microsoft Excel Software. Significance was determined based on $\mathrm{p}$-values as follows: one star if $\mathrm{p}$-value $<0.05$, two stars for $\mathrm{p}$-values $<0.01$, three stars to indicate $\mathrm{p}$-values $<0.001$, four stars indicate $\mathrm{p}$-values $<.0001$. All experimental numbers (n) are documented in figure legends. 


\section{Quantification Area, Surface area, and Volume}

Deconvolved image files were quantified in IMARIS. For in vivo imaging, each visible cell was manually selected based on the membrane reporter signal. Briefly, the construct surface and maskselection features in IMARIS were used to select a 3D space around each cell. The membrane reporter signal within the manually created surface was then used to render the surface structure the cell and the statistical analysis feature in IMARIS was used to export surface area and volume data. In vitro cell size information was generated using the same workflow, however, $2 \mu \mathrm{m}$ diameter beads embedded in the PA gels were used to standardize surface area and volume data. For a select set of studies (micropatterned PA gels), beads could not be embedded to serve as size standards. For these studies (X,Y) area is reported for the size of the cells. Correlation between $(\mathrm{X}, \mathrm{Y})$ area and 3D surface area and volume are provided in Figures 18D and 18E.

\section{Quantification of Cell Contacts}

Confocal images of genetically modified cells in vitro, were assessed for the number of cell contacts based on N-Cad IHC. Cells were plated at a density of $1 \times 10^{5}$ cells per $\mathrm{cm}^{2}$ to avoid multilayered cultures. Transfected cells were randomly selected based on fluorescent reporter signals and z-stacks were taken for the field of cells in the immediate area ( $\mathrm{n}=20$ images per condition). For each genetically modified cell, the number of N-Cad positive interfaces (puncta or ribbons) were manually counted to determine the number of cell contacts each cell made.

\section{Quantification of Calcium Transients}

Three 10 second acquisitions were recorded for each cell at $30 \mathrm{fps}$ on an Olympus FV3000RS confocal microscope. Using ImageJ V2.0.0 (NIH) an intensity profile of each cell per frame was plotted to generate a line graph. Each of the three series were analyzed for the number of calcium transient peaks and averaged to determine a mean rate $(\mathrm{Hz})$ per cell over the three series. A custom macro was written in excel to calculate time between calcium transient peaks for each imaging series and the deviation from the 
average interval per cell was used to calculate rhythmicity. Linear Regression (surface are/volume ration vs rate) was performed using (GraphPad Prism Software).

Quantification of N-Cadherin Junctional Intensity

Maximum intensity z-projections were generated for each cell pair using ImageJ V2.0.0 (NIH). A $5 \mu \mathrm{m}$ wide strip was then drawn through each junction (perpendicular to the plane of cell-cell contact) and the average intensity across the strip was calculated using the plot profile function in ImageJ. Intensity profiles were then exported to excel where regions $+/-10 \mu \mathrm{m}$ from the cell contact were quantified and the area under the curve was calculated. 


\section{REFERENCES}

Ahrens, J., Geveci, B., and Law, C (2005). Paraview: An End-User Tool for Large Data Visualization. Visualization Handbook: 717-731.

Arge E., Bruaset, A.M., Langtangen, H.P., Boston, M.A., Birkhäuser Boston: 163-202. Barry, A. K., H. Tabdili, I. Muhamed, J. Wu, N. Shashikanth, G. A. Gomez, A. S. Yap, C. J. Gottardi, J. de Rooij, N. Wang and D. E. Leckband (2014). " $\alpha$-catenin cytomechanics--role in cadherin-dependent adhesion and mechanotransduction." Journal of Cell Science 127(Pt 8): 17791791 .

Balay, S., S. Abhyankar, M. Adams, J. Brown, P. Brune, K. Buschelman, L. D. Dalcin, V. Eijkhout, W. Gropp, D. Kaushik, M. Knepley, D. May, L. C. McInnes, T. Munson, K. Rupp, P. Sanan, B. Smith, S. Zampini, H.

Balay, S., W. D. Gropp, L. C. McInnes and B. F. Smith (1997). Efficient Management of Parallelism in Object-Oriented Numerical Software Libraries. Modern Software Tools for Scientific Computing.

Boyett, M. R., H. Honjo, I. K. C. research and 2000 (2000). "The sinoatrial node, a heterogeneous pacemaker structure." academic.oup.com.

Bressan, M., T. Henley, J. D. Louie, G. Liu, D. Christodoulou, X. Bai, J. Taylor, C. E. Seidman, J. G. Seidman and T. Mikawa (2018). "Dynamic Cellular Integration Drives Functional Assembly of the Heart\&apos;s Pacemaker Complex." CELREP 23(8): 2283-2291.

Bressan, M., G. Liu and T. Mikawa (2013). "Early mesodermal cues assign avian cardiac pacemaker fate potential in a tertiary heart field." Science 340(6133): 744-748.

Bressan, M., P. B. Yang, J. D. Louie, A. M. Navetta, R. J. Garriock and T. Mikawa (2014). "Reciprocal myocardial-endocardial interactions pattern the delay in atrioventricular junction conduction." 141(21): 4149-4157.

Bressan, M. C., J. D. Louie and T. Mikawa (2014). "Hemodynamic Forces Regulate Developmental Patterning of Atrial Conduction." PLoS ONE 9(12): e115207-115220.

Chen, X., E. Koh, M. Yoder and B. M. Gumbiner (2009). "A Protocadherin-Cadherin-FLRT3 Complex Controls Cell Adhesion and Morphogenesis." PLoS ONE 4(12): e8411-8419. 
Chopra, A., E. Tabdanov, H. Patel, P. A. Janmey and J. Y. Kresh (2011). "Cardiac myocyte remodeling mediated by N-cadherin-dependent mechanosensing." American journal of physiology. Heart and circulatory physiology 300(4): H1252-H1266.

Christoffels, V. M., M. T. Mommersteeg, M. O. Trowe, O. W. Prall, C. de Gier-de Vries, A. T. Soufan, M. Bussen, K. Schuster-Gossler, R. P. Harvey, A. F. Moorman and A. Kispert (2006).

"Formation of the venous pole of the heart from an Nkx2-5-negative precursor population requires Tbx18." Circ Res 98(12): 1555-1563.

Courtemanche, M., R. J. Ramirez and S. Nattel (1998). "Ionic mechanisms underlying human atrial action potential properties: insights from a mathematical model." Am J Physiol 275(1): H301-321.

Egea, J., C. Erlacher, E. Montanez, I. Burtscher, S. YAMAGISHI, M. Hess, F. Hampel, R. Sanchez, M. T. Rodriguez-Manzaneque, M. R. Bosl, R. Fassler, H. Lickert and R. Klein (2008). "Genetic ablation of FLRT3 reveals a novel morphogenetic function for the anterior visceral endoderm in suppressing mesoderm differentiation." Genes \&amp; Development 22(23): 3349-3362.

Espinoza-Lewis, R. A., L. Yu, F. He, H. Liu, R. Tang, J. Shi, X. Sun, J. F. Martin, D. Wang, J. Yang and Y. Chen (2009). "Shox 2 is essential for the differentiation of cardiac pacemaker cells by repressing Nkx2-5." Developmental Biology 327(2): 376-385.

Fabbri, A., M. Fantini, R. Wilders and S. Severi (2017). "Computational analysis of the human sinus node action potential: model development and effects of mutations." J Physiol 595(7): 2365-2396.

Fast, V. G. and A. G. Kléber (1995). "Block of impulse propagation at an abrupt tissue expansion: evaluation of the critical strand diameter in 2- and 3-dimensional computer models." Cardiovascular Research 30(3): 449-459.

Forbes, M. S. and N. Sperelakis (1985). "Intercalated discs of mammalian heart: A review of structure and function." Tissue and Cell 17(5): 605-648.

Geuzaine, C. and J.-F. Remacle (2009). "Gmsh: A 3-D finite element mesh generator with built-in preand post-processing facilities." International Journal for Numerical Methods in Engineering 79(11): 1309-1331.

Glynn, P., B. Onal and T. J. Hund (2014). "Cycle length restitution in sinoatrial node cells: a theory for understanding spontaneous action potential dynamics." PLoS One 9(2): e89049. 
Goudy, J., T. Henley, H. G. Méndez and M. Bressan (2019). "Simplified platform for mosaic in vivo analysis of cellular maturation in the developing heart." Scientific Reports 9(1): 10716.

Grijalva, S. I., J. M. Gu, J. Li, N. Fernandez, J. Fan, J. H. Sung, S. Y. Lee, C. Herndon, E. M. Buckley, S. J. Park, F. H. Fenton and H. C. Cho (2019). "Engineered Cardiac Pacemaker Nodes Created by TBX18 Gene Transfer Overcome Source-Sink Mismatch." Adv Sci (Weinh) 6(22): 1901099.

Gutstein, D. E., F. Y. Liu, M. B. Meyers, A. Choo and G. I. Fishman (2003). "The organization of adherens junctions and desmosomes at the cardiac intercalated disc is independent of gap junctions." J Cell Sci 116(Pt 5): 875-885.

Hamburger, V. and H. L. Hamilton (1992). "A series of normal stages in the development of the chick embryo. 1951." Dev Dyn 195(4): 231-272.

Hand, P. E., B. E. Griffith and C. S. Peskin (2009). "Deriving macroscopic myocardial conductivities by homogenization of microscopic models." Bull Math Biol 71(7): 1707-1726.

Honjo, H., M. R. Boyett, S. R. Coppen, Y. Takagishi, T. Opthof, N. J. Severs and I. Kodama (2002). "Heterogeneous expression of connexins in rabbit sinoatrial node cells: correlation between connexin isotype and cell size." Cardiovascular Research 53(1): 89-96.

Honjo, H., M. R. Boyett, I. Kodama and J. Toyama (1996). "Correlation between electrical activity and the size of rabbit sino-atrial node cells." J Physiol 496 ( Pt 3): 795-808.

Hoogaars, W. M., A. Engel, J. F. Brons, A. O. Verkerk, F. J. de Lange, L. Y. Wong, M. L. Bakker, D. E. Clout, V. Wakker, P. Barnett, J. H. Ravesloot, A. F. Moorman, E. E. Verheijck and V. M. Christoffels (2007). "Tbx3 controls the sinoatrial node gene program and imposes pacemaker function on the atria." Genes Dev 21(9): 1098-1112.

Huveneers, S. and J. de Rooij (2013). "Mechanosensitive systems at the cadherin-F-actin interface." J Cell Sci 126(Pt 2): 403-413.

Inada, S., H. Zhang, J. O. Tellez, N. Shibata, K. Nakazawa, K. Kamiya, I. Kodama, K. Mitsui, H. I Dobrzynski, M. R. Boyett and H. Honjo (2014). "Importance of gradients in membrane properties and electrical coupling in sinoatrial node pacing." PLoS One 9(4): e94565.

Janssens, B., S. Goossens, K. Staes, B. Gilbert, J. van Hengel, C. Colpaert, E. Bruyneel, M. Mareel and F. Van Roy (2001). " $\alpha$ T-Catenin: a novel tissue-specific $\beta$-catenin-binding protein mediating strong cell-cell adhesion." Journal of Cell Science 114(17): 3177-3188. 
Jones, S. A., M. Yamamoto, J. O. Tellez, R. Billeter, M. R. Boyett, H. Honjo and M. K. Lancaster (2008). "Distinguishing Properties of Cells From the Myocardial Sleeves of the Pulmonary Veins: A Comparison of Normal and Abnormal Pacemakers." Circulation: Arrhythmia and Electrophysiology 1(1): 39-48.

Joyner, R. W. and F. J. van Capelle (1986). "Propagation through electrically coupled cells. How a small SA node drives a large atrium." Biophys J 50(6): 1157-1164.

Karaulanov, E., R. T. Böttcher, P. Stannek, W. Wu, M. Rau, S. Ogata, K. W. Y. Cho and C. Niehrs (2009). "Unc5B interacts with FLRT3 and Rnd1 to modulate cell adhesion in Xenopus embryos." PLoS ONE 4(5): e5742.

Keener, J. P. and J. Sneyd (1998). Mathematical physiology. New York, Springer. Kirchhof, C. J., F. I. Bonke, M. A. Allessie and W. J. Lammers (1987). "The influence of the atrial myocardium on impulse formation in the rabbit sinus node." Pflugers Arch 410(1-2): 198203.

Kirk, B. S., J. W. Peterson, R. H. Stogner and G. F. Carey (2006). "libMesh: a C++ library for parallel adaptive mesh refinement/coarsening simulations." Engineering with Computers 22(3): 237-254.

Kleber, A. G. and Y. Rudy (2004). "Basic mechanisms of cardiac impulse propagation and associated arrhythmias." Physiol Rev 84(2): 431-488.

Kostetskii, I., J. Li, Y. Xiong, R. Zhou, V. A. Ferrari, V. V. Patel, J. D. Molkentin and G. L. Radice (2005). "Induced deletion of the N-cadherin gene in the heart leads to dissolution of the intercalated disc structure." 96(3): 346-354.

Larson, E. D., J. Clair, W. S. P. o. the and 2013 (2013). "Depressed pacemaker activity of sinoatrial node myocytes contributes to the age-dependent decline in maximum heart rate." National Acad Sciences.

Lei, M. and P. Kohl (1998). "Swelling-induced decrease in spontaneous pacemaker activity of rabbit isolated sino-atrial node cells." Acta Physiol Scand 164(1): 1-12.

Li, J. (2005). "Cardiac-Specific Loss of N-Cadherin Leads to Alteration in Connexins With Conduction Slowing and Arrhythmogenesis." 97(5): 474-481. 
Li, J., S. Goossens, J. van Hengel, E. Gao, L. Cheng, K. Tyberghein, X. Shang, R. De Rycke, F. Van Roy and G. L. Radice (2012). "Loss of $\alpha \mathrm{T}$-catenin alters the hybrid adhering junctions in the heart and leads to dilated cardiomyopathy and ventricular arrhythmia following acute ischemia." Journal of Cell Science 125(4): 1058-1067.

Liu, Z., J. L. Tan, D. M. Cohen, M. T. Yang, N. J. Sniadecki, S. A. Ruiz, C. M. Nelson and C. S. Chen (2010). "Mechanical tugging force regulates the size of cell-cell junctions." Proceedings of the National Academy of Sciences of the United States of America 107(22): 9944-9949.

Lu, Y., T. N. James, S. Yamamoto and F. Terasaki (1993). "Cardiac conduction system in the chicken: Gross anatomy plus light and electron microscopy." The Anatomical Record 236(3): 493-510.

Luo, Y. (2003). "Cadherin-mediated adhesion is essential for myofibril continuity across the plasma membrane but not for assembly of the contractile apparatus." Journal of Cell Science 116(8): 1471-1479.

Luo, Y., M. Ferreira-Cornwell, H. Baldwin, I. Kostetskii, J. Lenox, M. Lieberman and G. Radice (2001). "Rescuing the N-cadherin knockout by cardiac-specific expression of N- or E-cadherin." Development 128(4): 459-469.

Lyashkov, A. E., M. Juhaszova, H. Dobrzynski, T. M. Vinogradova, V. A. Maltsev, O. Juhasz, H. A. Spurgeon, S. J. Sollott and E. G. Lakatta (2007). "Calcium Cycling Protein Density and Functional Importance to Automaticity of Isolated Sinoatrial Nodal Cells Are Independent of Cell Size." Circulation Research 100(12): 1723-1731.

Maretto, S., P.-S. Müller, A. R. Aricescu, K. W. Y. Cho, E. K. Bikoff and E. J. Robertson (2008). "Ventral closure, headfold fusion and definitive endoderm migration defects in mouse embryos lacking the fibronectin leucine-rich transmembrane protein FLRT3." Developmental Biology 318(1): 184-193.

Masson-Pevet, M., W. K. Bleeker and D. Gros (1979). "The plasma membrane of leading pacemaker cells in the rabbit sinus node. A qualitative and quantitative ultrastructural analysis." Circ Res 45(5): 621-629.

Masson-Pevet, M. A., W. K. Bleeker, E. Besselsen, B. W. Treytel, H. J. Jongsma and L. N. Bouman (1984). "Pacemaker cell types in the rabbit sinus node: a correlative ultrastructural and electrophysiological study." J Mol Cell Cardiol 16(1): 53-63 
Mavroidis, M., N. C. Athanasiadis, P. Rigas, I. Kostavasili, I. Kloukina, W. P. Te Rijdt, N. Kavantzas, D. Chaniotis, J. P. van Tintelen, I. Skaliora and C. H. Davos (2020). "Desmin is essential for the structure and function of the sinoatrial node: implications for increased arrhythmogenesis." Am J Physiol Heart Circ Physiol 319(3): H557-H570.

McDougal, R. A., T. M. Morse, T. Carnevale, L. Marenco, R. Wang, M. Migliore, P. L. Miller, G. M. Shepherd and M. L. Hines (2017). "Twenty years of ModelDB and beyond: building essential modeling tools for the future of neuroscience." J Comput Neurosci 42(1): 1-10.

Merkel, C. D., Y. Li, Q. Raza, D. B. Stolz and A. V. Kwiatkowski (2019). "Vinculin anchors contractile actin to the cardiomyocyte adherens junction." Mol Biol Cell 30(21): 2639-2650.

Mezzano, V., Y. Liang, A. T. Wright, R. C. Lyon, E. Pfeiffer, M. Y. Song, Y. Gu, N. D. Dalton, M. Scheinman, K. L. Peterson, S. M. Evans, S. Fowler, M. Cerrone, A. D. McCulloch and F. Sheikh (2016). "Desmosomal junctions are necessary for adult sinus node function." Cardiovasc Res 111(3): 274-286.

Miyamoto, T., L. Zhang, A. Sekiguchi, T. Hadama and T. Shimada (2002). "Structural differences in the cytoarchitecture and intercalated discs between the working myocardium and conduction $\mathrm{s}$ ystem in the human heart." Heart and Vessels 16(6): 232-240.

Mommersteeg, M. T., W. M. Hoogaars, O. W. Prall, C. de Gier-de Vries, C. Wiese, D. E. Clout, V. E. Papaioannou, N. A. Brown, R. P. Harvey, A. F. Moorman and V. M. Christoffels (2007).

"Molecular pathway for the localized formation of the sinoatrial node." Circ Res 100(3): 354-362.

Monfredi, O., K. Tsutsui, B. Ziman, M. D. Stern, E. G. Lakatta and V. A. Maltsev (2018). "Electrophysiological heterogeneity of pacemaker cells in the rabbit intercaval region, including the SA node: insights from recording multiple ion currents in each cell." Am J Physiol Heart Circ Physiol 314(3): H403-H414.

Müller, P.-S., R. Schulz, S. Maretto, I. Costello, S. Srinivas, E. Bikoff and E. Robertson (2011). "The fibronectin leucine-rich repeat transmembrane protein Flrt2 is required in the epicardium to promote heart morphogenesis." 138(7): 1297-1308.

Nagafuchi, A., S. Ishihara and S. Tsukita (1994). "The roles of catenins in the cadherin-mediated cell adhesion: functional analysis of E-cadherin-alpha catenin fusion molecules." The Journal of Cell Biology 127(1): 235-245.

Nikolaidou, T., O. V. Aslanidi, H. Zhang and I. R. Efimov (2012). "Structure-function relationship in the sinus and atrioventricular nodes." Pediatr Cardiol 33(6): 890-899. 
Ogata, S., J. Morokuma, T. Hayata, G. Kolle, C. Niehrs, N. Ueno and K. W. Y. Cho (2007). "TGF-beta signaling-mediated morphogenesis: modulation of cell adhesion via cadherin endocytosis." Genes \&amp; Development 21(14): 1817-1831.

Ong, L. L., N. Kim, T. Mima, L. Cohen-Gould and T. Mikawa (1998). "Trabecular myocytes of the embryonic heart require N-cadherin for migratory unit identity." Dev Biol 193(1): 1-9.

Opthof, T. (1988). "The mammalian sinoatrial node." Cardiovascular Drugs and Therapy 1(6): 573-597.

Rohr, S., J. P. Kucera, V. G. Fast and A. G. Kléber (1997). "Paradoxical improvement of impulse conduction in cardiac tissue by partial cellular uncoupling." Science 275(5301): 841-844.

Rohr, S., J. P. Kucera and A. G. KLÉBER (1998). "Slow Conduction in Cardiac Tissue, I: Effects of a Reduction of Excitability Versus a Reduction of Electrical Coupling on Microconduction." Circulation Research 83(8): 781-794.

Rossi, S. and B. E. Griffith (2017). "Incorporating inductances in tissue-scale models of cardiac electrophysiology." Chaos 27(9): 093926.

Satoh, H., L. M. Delbridge, L. A. Blatter and D. M. Bers (1996). "Surface:volume relationship in cardiac myocytes studied with confocal microscopy and membrane capacitance measurements: speciesdependence and developmental effects." Biophys J 70(3): 1494-1504.

Shaw, R. M., A. J. Fay, M. A. Puthenveedu, M. von Zastrow, Y. N. Jan and L. Y. Jan (2007). "Microtubule plus-end-tracking proteins target gap junctions directly from the cell interior to adherens junctions." Cell 128(3): 547-560.

Shaw, R. M. and Y. Rudy (1997). "Ionic mechanisms of propagation in cardiac tissue. Roles of the sodium and L-type calcium currents during reduced excitability and decreased gap junction coupling." Circulation Research 81(5): 727-741.

Shimada, T., H. Kawazato, A. Yasuda, N. Ono and K. Sueda (2004). "Cytoarchitecture and intercalated disks of the working myocardium and the conduction system in the mammalian heart." Anat Rec A Discov Mol Cell Evol Biol 280(2): 940-951.

Smyth, J. W., T. T. Hong, D. Gao, J. M. Vogan, B. C. Jensen, T. S. Fong, P. C. Simpson, D. Y. Stainier, N. C. Chi and R. M. Shaw (2010). "Limited forward trafficking of connexin 43 reduces cell-cell coupling in stressed human and mouse myocardium." J Clin Invest 120(1): 266-279. 
Swope, D., L. Cheng, E. Gao, J. Li and G. L. Radice (2012). "Loss of cadherin-binding proteins betacatenin and plakoglobin in the heart leads to gap junction remodeling and arrhythmogenesis." Mol Cell Biol 32(6): 1056-1067.

Tomás, A. R., A. C. Certal and J. Rodríguez-León (2011). "FLRT3 as a key player on chick limb development." Developmental Biology 355(2): 324-333.

Unudurthi, S. D., R. M. Wolf, T. H. F. i. physiology and 2014 (2014). "Role of sinoatrial node architecture in maintaining a balanced source-sink relationship and synchronous cardiac pacemaking." frontiersin.org.

van Eif, V. W. W., S. Stefanovic, K. van Duijvenboden, M. Bakker, V. Wakker, C. de Gier-de Vries, S. Zaffran, A. O. Verkerk, B. J. Boukens and V. M. Christoffels (2019). "Transcriptome analysis of mouse and human sinoatrial node cells reveals a conserved genetic program." Development (Cambridge, England) 146(8): dev173161-173129.

Vedantham, V. (2015). "New Approaches to Biological Pacemakers: Links to Sinoatrial Node Development." Trends in Molecular Medicine 21(12): 749-761.

Vedantham, V., G. Galang and M. Evangelista (2015). "RNA sequencing of mouse sinoatrial node reveals an upstream regulatory role for Islet-1 in cardiac pacemaker cells." Circulation.

Vite, A., C. Zhang, R. Yi, S. Emms and G. L. Radice (2018). " $\alpha$-Catenin-dependent cytoskeletal tension controls Yap activity in the heart." Development (Cambridge, England) 145(5): dev149823.

Wickline, E. D., I. W. Dale, C. D. Merkel, J. A. Heier, D. B. Stolz and A. V. Kwiatkowski (2016). "aTCatenin Is a Constitutive Actin-binding $\alpha$-Catenin That Directly Couples the Cadherin Catenin Complex to Actin Filaments." The Journal of biological chemistry 291(30): 15687-15699.

Wiese, C., T. Grieskamp, R. Airik, M. T. Mommersteeg, A. Gardiwal, C. de Gier-de Vries, K. SchusterGossler, A. F. Moorman, A. Kispert and V. M. Christoffels (2009). "Formation of the sinus node head and differentiation of sinus node myocardium are independently regulated by Tbx 18 and Tbx3." Circ Res 104(3): 388-397.

Ye, W., J. Wang, Y. Song, D. Yu, C. Sun, C. Liu, F. Chen, Y. Zhang, F. Wang, R. P. Harvey, L. Schrader, J. F. Martin and Y. Chen (2015). "A common Shox2-Nkx2-5 antagonistic mechanism primes the pacemaker cell fate in the pulmonary vein myocardium and sinoatrial node." Development 142(14): 2521-2532. 
Yonemura, S., M. Itoh, A. Nagafuchi and S. Tsukita (1995). "Cell-to-cell adherens junction formation and actin filament organization: similarities and differences between non-polarized fibroblasts and polarized epithelial cells." J Cell Sci 108 ( Pt 1): 127-142.

Yonemura, S., Y. Wada, T. Watanabe, A. Nagafuchi and M. Shibata (2010). "alpha-Catenin as a tension transducer that induces adherens junction development." Nature Cell Biology 12(6): 533-542.

Zemljic-Harpf, A., A. M. Manso and R. S. Ross (2009). "Vinculin and talin: focus on the myocardium." J Investig Med 57(8): 849-855.

Zhang, H., A. V. Holden, I. Kodama, H. Honjo, M. Lei, T. Varghese and M. R. Boyett (2000). "Mathematical models of action potentials in the periphery and center of the rabbit sinoatrial node." Am J Physiol Heart Circ Physiol 279(1): H397-421.

Zhang and H. Zhang (2017). PETSc Users Manual Revision 3.8, ; Argonne National Lab. (ANL), Argonne, IL (United States): Medium: ED; Size: 264 p. 


\section{CHAPTER 4: CONCLUSION AND FURTURE DIRECTIONS}

\section{Conclusion}

This dissertation work on AJ regulation in the developing SAN has developed a better understanding of CPC cellular dynamics by providing insight into the mechanisms that regulate CPC morphology and function. Specifically, these studies have opened new areas of investigation into how cell shape, cell size, and cell-cell interactions are regulated in CPCs of the SAN.

Interestingly, this dissertation research has led to the identification of a possible therapeutic molecule in treating SAN dysfunction. FLRT3 has been identified as an important cadherin antagonist in the SAN. When FLRT3 dynamics were manipulated in the SAN and atria changes in cell morphology and junctional dynamics were observed. Thus, FLRT3 is an effector molecule controlling cell clustering, cell size, and cell coupling. As previously described when FLRT3 was overexpressed in the atria cells lost the ability to form robust AJs. Since AJs are sufficient for gap junction protein trafficking when FLRT3 diminishes NCad, one might expect, gap junction electrical coupling will be diminished as well.

Maintaining a unique small cell size and low coupling is beneficial for CPC function in firing spontaneous action potentials, as previously discussed. Current regenerative medicine for treating SAN dysfunction is centered around developing a biological pacemaker. Currently biological pacemakers lack stability thus, this form of treatment has not reached the stage of clinical trials. Given the regulatory dynamics of FLRT3 in CPC structure and morphology, FLRT3 is an excellent candidate molecule for maintaining CPC integrity in a biological pacemaker system. FLRT3, might be the missing factor in SAN regenerative medicine. As mentioned in the introductory chapter cell biology, genetics, and physiology all play a vital role in producing the unique cell type, CPCs. SAN regenerative medicine has focused on the transcriptional landscape to make biological pacemakers. When a cell biology effector molecule like 
FLRT3 is introduced along with the genetics and physiology constructs of biological pacemakers, this may complete the work. Thus, producing stable biological pacemakers to be used in regenerative medicine to treat diseases that lead to SAN dysfunction.

Moving forward, although the current graduate studies have made many innovative discoveries several outstanding questions remain. Specifically, our findings raise the question of how do AJ manipulations influence genetics and does force mediated AJ engagement regulate genes associated with CPC function? Thus, future directions should explore the interplay between CPC cell biology and genetics. It makes sense now to directly ask to what extent CPCs can sense variations in local biomechanics and how mechanical inputs are translated to changes in transcription. Towards these topics, the avian model of heart development is still a relevant and powerful system to explore these topics

As shown previously we have tested the hypothesis that the unique cellular architecture required for CPC function is patterned via dynamic regulation of $\mathrm{AJ}$ engagement during developmental assembly of the sinoatrial node. We have demonstrated both the necessity and sufficiency of AJ regulation for CPC structural patterning. The logical extensions from this dissertation work will now be discussed. The first of these is to explore the downstream consequences of $\mathrm{AJ}$ regulation on the molecular pattering of the CPC-lineage, and the second is to define the effector molecules that relay information regarding the extent of AJ engagement to the cell interior/nucleus. Given the available tool sets in the avian model of heart development outlined previously, these proposed future studies could be successfully executed.

\section{Future Directions}

Our current data, indicate that loss of $\mathrm{AJ}$ formation is required for patterning the appropriate $\mathrm{CPC}$ morphology and that ectopic introduction of AJ disrupts the ability of CPCs to rhythmically initiate electrical impulses. Given that the AJ plays an integral part in eliciting mechanotransducive signaling cues, our data now support a hypothesis in which at least a subset of the molecular features that have previously been thought to simply represent fixed characteristics of the CPC lineage, may be dynamically regulated by local biomechanics. I propose to test this hypothesis as follows: using the published protocol 
for mosaic overexpression or ablation of the $\mathrm{AJ}$ protein, $\mathrm{N}$-Cadherin, in the embryonic heart. In the former chapter this technique was used to create volumetric renderings of the cells for morphometric analysis, one could extend these experiments to conduct molecular characterization.

In short, embryonic avian hearts will be transfected during early looping stages with integrating DNA constructs encoding either membrane targeted TagRFP and a nuclear target GFP (as controls), a Ncadherin expressing construct (polycistronic membrane TagRFP-2a-N-Cad) or an N-Cadherin blocking construct (polycistronic membrane TagRFP-2a-N-CadD116-624) (Goudy et al., 2019). In addition, to the $\mathrm{N}$-cadherin overexpressing or blocking construct hearts will be transfection with a Megakaryoblastic leukemia protein-1 (MKL1) expressing construct fused to monomeric GFP ( MKL1-GFP). The MKL1 expressing construct was designed in our lab to be used as a stretch reporter to assay changes in AJmediated mechanobiology. MKL1 is also known as Myelin and lymphocyte protein (MAL). MKL1/MAL is a co-activator of serum response factor (SRF) activity. MKL1 binds to cytoplasmic G-actin, when actin polymerization (F-actin) occurs there is less G-actin in the cytoplasm and MKL1 moves into the nucleus because it is a SRF transcriptional co-factor (Miralles et al., 2003). Since the process of actinpolymerization occurs in response to increased mechanical strain, we predict that atria myocytes will show more nuclear accumulation of MKL1, because mature AJs lead to higher forces and is associated with actin-polymerization (Yeung et al., 2005; Gupta et al., 2016; Chopra et al., 2018). Thus, we can assay, in real-time, the relative tension that CPCs and atrial myocytes sense via MKL1 localization patterns.

The major goals of using these constructs are to disrupt the AJ and assess changes in AJmechanobiology in genetically modified cells. Transfected hearts will be allowed to develop through the completion of cardiac morphogenesis ( $~ 144$ hours post transfection). Hearts will then be isolated and CPC myocardium, atrial myocardium, and ventricular myocardium will be mechanically dissected. Cells from each region will then be enzymatically dissociated and transfected cells will be collected using Fluorescence Activated Cell Sorting (FACS) according to our previously published protocols (Goudy et 
al., 2019). As the polycistronic construct is based on the viral-2a system, fluorescence intensity can be used to normalize the level of N-Cadherin or N-Cadherin D116-624 expression per cell. From each tissue sample three groups of FACS sorted cells will be collected, high expressing, low expressing, and no expression. Approximately 50,000 cells per condition/tissue will be collected and then processed for cDNA library construction. It is estimated that this will require 8-10 hearts per sample and all samples will be replicated in triplicate ( $24-30$ hearts total).

Following cDNA synthesis, we will use a targeted real-time PCR approach to explore how overexpression and blocking AJ engagement in vivo influences both positive and negative functional markers of the CPC lineage including the ion channels (Hcn4, Cacna1h, Cacna1g, Cacna1g, and Scn5a), gap junctions (Gja1 and Gja5), and transcription factors (Tbx3, Tbx18, Shox2 and Is11) in each region of the heart. Gene expression will be compared directly to age matched control CPC, atrial, and ventricular myocardium. This analysis will provide the first insights into how functional markers of the CPC-lineage respond to altered $\mathrm{AJ}$ mechanics.

In addition to the candidate approach outlined above, we will also use unbiased RNA sequencing to assess global changes in transcriptional changes. As above primary embryonic cardiomyocytes will be transfected in vivo and cells will be sorted following $\sim 144$ hours of developmental maturation. RNA will be isolated from sorted cell population and prepared for Click Ligation Next Generation Sequencing (ClickSeq). For this analysis, highly expressing control, N-Cadherin, and N-cadherin D116-624 transfected atrial and ventricular cells (based on FACS) as well as wildtype untransfected control cells (CPC/atrial/ventricular) will be sequenced (2 biological replicates per group). Principle component analysis (PCA) based on differential gene expression will then be used to identify the ability of AJ modulation to alter molecular fate towards or away from native cell states (CPCs, Atrial, and Ventricular myocytes). This analysis will also define the genetic pathways altered by N-Cadherin downstream signaling. This relatively straight forward approach will provide a global and unbiased evaluation of the genetic pathways controlled via AJ-mechanobiology within the embryonic myocardium. Importantly, 
having now established that N-Cadherin does, in fact, regulate CPC morphology, the above studies represent the next logical set line of investigation. Completion of these experiments will define the molecular consequences of AJ regulation within embryonic PCs

It is important to understand what transmitting molecules play a role in AJ-mediated mechanobiology in CPCs, because this is currently unknown. The role of NCad-based AJs has been well documented and studied in the WM. In addition to the literature, this current graduate work has given the first account of AJ regulation throughout $\mathrm{CPC}$ development. It is currently known that AJs are sufficient to drive gap junction and intercalated discs formation in the WM (ventricles and atrias) (Kostetskii et al., 2005; Li et al., 2008). Intercalated discs are intracellular and extracellular structures comprised of a variety of junctions stacked on top of each other at the cell-cell interface (Shimada et al., 2004; Severs, 2007; Kleber and Saffitz, 2014; Asimaki et al., 2015; Leo-Macias et al., 2016; Moncayo-Arlandi and Brugada, 2017; Vermij et al., 2017; Gourdie, 2019). In fact, connexins and NCad facilitate electromechanical coupling between cardiomyocytes of the WM. Mechanical and electrical coupling in the WM is dependent on AJ and gap junction formation respectively. (Gourdie 2019; Merkel et al., 2019). When NCad is depleted in WM cells major consequences arise. The same rule applies for depletion of gap junctions in the WM (Li et al., 2008). There is a vast amount of knowledge on what structures and proteins form an AJ but how the AJ influences the cell internally is poorly understood.

For instance, the AJ is comprised of extracellular proteins NCad, and intracellular proteins BCat, aTCat, p120 that link the AJ to the cell cytoskeleton (Reynolds et al., 1994; Peifer et al., 1994). This produces a mechanotransduction response at the cellular level. Extracellular tractional force in cardiomyocytes is perceived through AJs through muscle contraction. In general, components of the AJ complex are thought of as mechanosensors. However, which molecules transmit the signals in CPCs are unknown. Thus, which proteins and genes in mechanosensing pathways, relay the biochemical signals are that contribute to CPC patterning need to be identified. Mechanosensing is known to influence the 
structure and function of cells, therefore, it makes sense as to why mechanosensing in CPCs should be well understood since CPC structure and function must be tightly regulated.

To determine whether the signals being transmitted when an $\mathrm{AJ}$ forms in embryonic cardiomyocytes (that influence cell size) are primarily being transmitted through the actomyosin cytoskeleton, intermediate filament system, or microtubule cytoskeleton. Using our previously discussed protocol for mosaic in vivo gene knockdown using shRNA (Goudy et al., 2019), we will screen components of the AJ complex that tether to actin (aT-Catenin, b-catenin, p120-catenin, and vinculin), Desmosome proteins that tether to intermediate filaments (Desmoglein and Desmoplakin), and microtubule associated proteins (EB1 and Bin1/2). Briefly, shRNAs designed against the primary chick sequences of the above genes will be designed and subcloned into an integrating DNA expression construct. The constructs will be transfected into hearts at early cardiac looping stages and embryos will be allowed to develop for $\sim 72$ hours. Transfected cells will be sorted as described above and RT-PCR will be used to confirm target gene knockdown.

Following initial validation, the same protocol will be used, however, transfected hearts will be allowed to develop for $\sim 144$ hours (through the completion of cardiac morphogenesis). Hearts will then be fixed and processed for imaging. Individual cells will be scanned, and full volumetric reconstructions will be generated as described previously. At least 20 cells per group, from 3 biological replicates will be used for analysis. Cell surface area, volume, and aspect ratio will be used to determine whether knockdown influenced cytoarchitecture. All data will be compared with equivalent studies already conducted using $\mathrm{N}$-cadherin disruption to determine which cytoskeleton system is transmitting information downstream cell-cell coupling. These data will establish the first information regarding how differential AJ engagement is sensed by CPCs and WM to influence cell morphology. Fulfilling the central goal of these future proposed studies, to identify the effector molecules that transmit information regarding the extent of $\mathrm{AJ}$ engagement to the cell interior. 
As described in Chapter 3, we identified a novel factor enriched in the forming SAN, FLRT3. FLRT3 is a transmembrane protein capable of autonomously regulating myocardial cell size. Previous studies using xenopus gastrulation as a model system (Chen et al., 2019) had indicated that FLRT3 may act as a negative regulator of classical cadherin function. Therefore, we hypothesized in this dissertation work that FLRT3 functions to disrupt N-cadherin mediated AJ formation in the forming SAN. Thus far, our data has confirmed this hypothesis and we have quantitatively demonstrated a disruption in NCadherin function in FLRT3 overexpression studies. In addition to its function as a negative regulator of cadherin function, FLRT3 has also been identified as a regulator of homotypic cell sorting and excitatory synapse formation (Del Toro et al., 2017; Leyva-Diaz et al., 2014). These functions of FLRT3 may explain the ability of CPCs to tightly cluster in the absence of $\mathrm{AJ}$ formation. Therefore, we propose that we will examine that ability of FLRT3 to induce cell sorting/clustering in primary embryonic myocytes. Importantly, if it can be demonstrated that FLRT3 has both the ability to control cell size and cell clustering it would dramatically expediate the process of rational design for generating CPC-like cells for therapeutic purposes. Expression of this single factor could be used to control multiple, critical aspects of the engineered cells. Therefore, the proposed studies are a natural and important extension of the completed experiments outlined in this dissertation research study.

We examined previously which domains of FLRT3 (if any) regulated cardiac AJ formation. We have identified that series of basic amino acids on the cytoplasmic tail of the protein are required for Ncadherin regulation. This domain is predicted to interact with several GTPases. To expand on this study, we will explore whether the extracellular domains of FLRT3 are required for cell sorting/clustering. We will generate several FLRT3 domain mutants and subclone them into our previously described integrating DNA constructs (Goudy et al., 2019). These constructs will be transfected into the embryonic heart at early looping stages as described above. Following incubation through the completion of cardiac morphogenesis ( $\sim 144$ hours), hearts will be fixed and imaged. Our data indicate that cells that express wildtype FLRT3 tightly cluster in both the atrial and ventricular working myocardium. Therefore, our 
extracellular domain mutant constructs will be compared to control and FLRT3 overexpression constructs to demonstrate which region(s) of the extracellular domain of FLRT3 is required for clustering. As above full volumetric reconstructions of individual cells will be constructed ( 20 cells per condition, 6 biological replicates). These data will identify if the extracellular domain of FLRT3 has a function independent from the cytoplasmic regions' regulation of AJ formation. Furthermore, as several protein-protein interactions have been mapped to the extracellular region of FLRT3, these studies will inform us as to which interacting partners may play an as of yet undefined role in CPC patterning. This will determine if the extracellular domain of FLRT3 controls PC cell clustering.

As described above FLRT3 has been implicated in excitatory synapse formation (Del Toro et al., 2017; Leyva-Diaz et al., 2014). As an extension of our studies, we will explore whether FLRT3 expression influences the localization of proteins associated with additional cell-cell junctions and/or channel localization. Of particular interest, the goal of these studies will be to track gap junction protein localization following FLRT3 overexpression or knockdown. These data will identify whether FLRT3 has the ability to cell autonomously control the extent of electrical coupling among PCs.

As above, our wild-type and domain mutant versions of FLRT3 will be transfected into the heart at looping stages. Following 72 hours of culture, hearts will be isolated and dissociated. Myocytes will then be plated on coverslips at low density and immunohistochemistry will be used to track the localization of two gap junction proteins, $\mathrm{Cx} 40$ and $\mathrm{Cx} 43$. Cell pairs that form in culture will be imaged with a Zeiss 800 laser scanning confocal microscopy fitted with an Airyscan super-resolution detection system. $\mathrm{Cx} 40$ and $\mathrm{Cx} 43$ localization will then be quantified based on signal intensity at cell-cell contacts. This analysis will provide the first insight into whether FLRT3 protein levels influence electrical coupling among myocytes, determining if FLRT3 influences cell excitability

Importantly, all the approaches outlined above have been developed, validated, and/or published by our group. These continuing experiments are feasible and are of high importance to CPC biology. The experimental approaches proposed for these studies were specifically designed such that any data 
collected will be of immediate utility regardless of whether it agrees with our overall hypothesis. For instance, if our data demonstrate that major, or a subset, genetic pathways relevant to CPC physiology are sensitive to AJ dynamics, this would provide the first proof-of-principle that cellular mechanics need to be considered in order to build a comprehensive understanding of CPC development and/or disease. Conversely, if our data demonstrates little or no influence on molecular cell fate, it would indicate that cellular mechanics can be viewed as negligible in CPC genetic patterning (though not for morphological patterning). This would also be a significant finding for the field, suggesting that future approaches that we and others take to create genetically engineered CPCs for therapeutic purposes can largely focus on genetic control and morphological control as separate variables without much cross talk between the two. Similarly, if removal of one or any component of the actin, intermediate, or microtubule cytoskeleton anchoring complexes phenocopy N-Cadherin disruption it will provide the first insights into the mechotransduction pathway(s) that are responsible for CPC morphological patterning.

In reference to the FLRT3 studies, currently almost nothing is known regarding FLRT3 function in the heart. Our data implicate this molecule as an extremely exciting factor cable of directly controlling cardiomyocyte morphology. This alone would highlight FLRT3 as a potentially useful factor for future regenerative medicine or tissue engineering approaches for creating cell-based therapies in the heart. A natural extension of the initial studies is, therefore, to evaluate the total scope of FLRT3 function in cardiomyocytes. If our data demonstrates that FLRT3 can induce cell clustering and/or influence gap junctional coupling, these data would further strengthen the utility of FLRT3 as a clinically relevant target. However, if the proposed studies described above demonstrate that FLRT3 functions only as an AJ regulator, additional strategies will need to be developed to control clustering and/or coupling in order for future tissue-engineered CPC-like cells to effectively control heart rate.

As outlined in the justification of these proposed future directions, mechanobiology in the heart, particularly in CPCs, is poorly understood yet represents an exciting and critical direction to explore. Through the course of this dissertation work, we have developed new tools and approaches to directly 
assay mechanical influences on CPC maturation. Also, we have identified FLRT3 is a new factor relevant to SAN function. Moving forward a major area of investigation should be to link the cell biological concepts described in this work with the genetic and physiological principals that dictate pacemaker function. 


\section{REFERENCES}

Asimaki, A., Kleber, A. G., \& Saffitz, J. E.. (2015). Pathogenesis of Arrhythmogenic Cardiomyopathy. Canadian Journal of Cardiology, 31(11), 1313-1324. https://doi.org/10.1016/j.cjca.2015.04.012 Chen, X., et al., (2009). A Protocadherin-Cadherin-FLRT3 Complex Controls Cell Adhesion and Morphogenesis. PLoS ONE. 4(12): p. e8411-9.

Chopra, A., Kutys, M. L., Zhang, K., Polacheck, W. J., Sheng, C. C., Luu, R. J., Eyckmans, J., Hinson, J. T., Seidman, J. G., Seidman, C. E., \& Chen, C. S. (2018). Force Generation via $\beta$-Cardiac Myosin, Titin, and $\alpha$-Actinin Drives Cardiac Sarcomere Assembly from Cell-Matrix Adhesions. Developmental cell, 44(1), 87-96.e5. https://doi.org/10.1016/j.devcel.2017.12.012

Del Toro, D., et al., (2017). Regulation of Cerebral Cortex Folding by Controlling Neuronal Migration via FLRT Adhesion Molecules. Cell. 169(4): p. 621-635.e16.

Goudy, J., et al., (2019). Simplified platform for mosaic in vivo analysis of cellular maturation in the developing heart. Scientific Reports. 9(1): p. 10716.

Gourdie RG. (2019). The Cardiac Gap Junction has Discrete Functions in Electrotonic and Ephaptic Coupling. Anat Rec (Hoboken). 302(1):93-100. doi: 10.1002/ar.24036.

Gupta, M., Doss, B., Lim, C. T., Voituriez, R., \& Ladoux, B. (2016). Single cell rigidity sensing: A complex relationship between focal adhesion dynamics and large-scale actin cytoskeleton remodeling. Cell adhesion \& migration, 10(5), 554-567. https://doi.org/10.1080/19336918.2016.1173800

Kleber, A. G., \& Saffitz, J. E. (2014). Role of the intercalated disc in cardiac propagation and arrhythmogenesis. Frontiers in physiology, 5, 404. https://doi.org/10.3389/fphys.2014.00404

Kostetskii, I., Li, J., Xiong, Y., Zhou, R., Ferrari, V. A., Patel, V. V., Molkentin, J. D., \& Radice, G. L. (2005). Induced deletion of the N-cadherin gene in the heart leads to dissolution of the intercalated disc structure. Circulation research, 96(3), 346-354.

https://doi.org/10.1161/01.RES.0000156274.72390.2c

Leyva-Díaz, E., et al., (2014). FLRT3 is a Robo1-interacting protein that determines Netrin-1 attraction in developing axons. Current biology: CB. 24(5): p. 494-508. 
Leo-Macias, A., Agullo-Pascual, E., Sanchez-Alonso, J. L., Keegan, S., Lin, X., Arcos, T., Feng-XiaLiang, Korchev, Y. E., Gorelik, J., Fenyö, D., Rothenberg, E., Rothenberg, E., \& Delmar, M. (2016). Nanoscale visualization of functional adhesion/excitability nodes at the intercalated disc. Nature communications, 7, 10342. https://doi.org/10.1038/ncomms10342

Li, J., Levin, M.D., Xiong, Y., Petrenko, N., Patel, V.V., Radice, G.L. (2008). N-cadherin haploinsufficiency affects cardiac gap junctions and arrhythmic susceptibility. J Mol Cell Cardiol. 44(3):597-606. doi: 10.1016/j.yjmcc.2007.11.013.

Merkel, C. D., Li, Y., Raza, Q., Stolz, D. B., \& Kwiatkowski, A. V.. (2019). Vinculin anchors contractile actin to the cardiomyocyte adherens junction. Molecular Biology of the Cell, 30(21), 2639-2650. https://doi.org/10.1091/mbc.e19-04-0216

Miralles, F., Posern, G., Zaromytidou, A.-I., \& Treisman, R.. (2003). Actin Dynamics Control SRF Activity by Regulation of Its Coactivator MAL. Cell, 113(3), 329-342. https://doi.org/10.1016/s00928674(03)00278-2

Moncayo-Arlandi, J., \& Brugada, R. (2017). Unmasking the molecular link between arrhythmogenic cardiomyopathy and Brugada syndrome. Nature reviews. Cardiology, 14(12), 744-756. https://doi.org/10.1038/nrcardio.2017.103

Peifer, M., Berg, S., Reynolds, A.B. (1994). A repeating amino acid motif shared by proteins with diverse cellular roles. Cell. 76:789-791.

Reynolds, A.B., Daniel, J., McCrea, P.D., Wheelock, M.J., Wu, J., Zhang, Z. (1994). Identification of a new catenin: the tyrosine kinase substrate p120cas associates with E-cadherin complexes. Mol Cell Biol. 14:8333-42.

Severs, N. J.. (2007). The Carboxy Terminal Domain of Connexin43. Circulation Research, 101(12), 1213-1215. https://doi.org/10.1161/circresaha.107.165662

Shimada, T., Kawazato, H., Yasuda, A., Ono, N., \& Sueda, K. (2004). Cytoarchitecture and intercalated disks of the working myocardium and the conduction system in the mammalian heart. The Anatomical Record, 280A(2), 940-951. https://doi.org/10.1002/ar.a.20109

Vermij, S. H., Abriel, H., \& van Veen, T. A. (2017). Refining the molecular organization of the cardiac intercalated disc. Cardiovascular research, 113(3), 259-275. https://doi.org/10.1093/cvr/cvw259 
Yeung, T., Georges, P. C., Flanagan, L. A., Marg, B., Ortiz, M., Funaki, M., Zahir, N., Ming, W., Weaver, V., \& Janmey, P. A. (2005). Effects of substrate stiffness on cell morphology, cytoskeletal structure, and adhesion. Cell motility and the cytoskeleton, 60(1), 24-34. https://doi.org/10.1002/cm.20041 


\section{APPENDIX: MICROINJECTION-BASED SYSTEM FOR IN VIVO IMPLANTATION OF EMBRYONIC CARDIOMYOCYTES IN THE AVIAN EMBRYO ${ }^{3}$}

\section{Introduction}

Cardiac developmental research has benefitted enormously from the advent of germline transgenic model systems which have identified many of the gene regulatory networks that pattern different cell lineages and functional domains in the heart. However, identifying how these gene networks interact with and respond to microenvironmental conditions, including paracrine/juxtacrine signals and biophysical inputs (stretch, strain, hemodynamic flow), can be challenging. As such, it is not always easy to determine whether a cellular phenotype arises as a direct consequence of a genetic perturbation or as a secondary result of an adaption to changes in cardiac biomechanics or higher order tissue composition (Guo and Pu,2018; Guo et al., 2017).

Grafting experiments, which have classically been used to address concepts of fate specification, commitment, induction, and competence (Rugh, 1962; Slack, 2013) would represent an ideal approach to circumvent some of the challenges inherent in defining cell autonomous versus environmental influence in the heart. Unfortunately, heart contractions make standard grafting approaches difficult. Rapid movement of the tissue often prevents grafted cells from adhering to the heart and large tissue punctures (normally required for grafting) frequently lead to heart failure and embryonic lethality (Reinecke et al., 1999; Rojas et al., 2017; Zhang et al., 2001). Therefore, we have developed a pressure- based, microinjection system for precision cellular implantation into the developing chick heart, circumventing the technical hurdles of tissue grafting described previously (Bressan et al., 2013; Bressan et al., 2014). Using this technique, individual or small groups of cardiac cells isolated from a donor embryo can be microinjected into a variety of regions of a host embryonic heart eliminating the need for extensive host preparation and the large tissue insults that arise using standard grafting techniques. The microinjection

\footnotetext{
${ }^{3}$ This apendix previously appeared as an article in the Journal of Visualized Experiments. The original citation is as follows: Henley T, Thomas K, Bressan M. Microinjection-based System for In Vivo Implantation of Embryonic Cardiomyocytes in the Avian Embryo. Journal of Visualized Experiments. 02/2019;17:144. doi: 10.3791/59267.
} 
needles used for these implantation studies have an outer diameter of $\sim 30-40 \mu \mathrm{m}$, which means that the needle can be placed directly in the target tissue (i.e., can penetrate the embryonic myocardial wall) and cells can be focally delivered with minimal damage to the surrounding tissue. The protocol can be used to perform a variety of isotopic, heterotopic, isochoric, and heterochronic manipulations, providing a rapid, flexible, and low-cost approach to directly examine classical experimental embryological paradigms in the developing four-chambered heart.

In the protocol outlined below, we label donor cells with a cell permeant fluorescent dye, which allows for the success of a microinjection experiment to be monitored in real-time and the location of engrafted cells to be documented without the need for any additional staining. However, it should be noted that this approach is best suited for short term experiments (approximately $48 \mathrm{~h}$ ) as the fluorescent dye can be lost through cell division. Alternative approaches can be used for longer term experiments.

While we are presenting this technique in the context of cardiac development, we have used it to great effect for cell implantation experiments into the mesoderm, head, limbs, and somites. As such the basic approach described below is highly tractable and can be used in a variety of organ systems.

\section{Protocol}

All methods described adhere to animal care guidelines of The University of North Carolinaat Chapel Hill.

\section{Preparation of micro-injection pipettes}

a. Pull glass capillaries using a micropipette puller. For some injections, needle beveling is recommended as it provides an extremely sharp surface devoid of structural impurities. To do this, polish the end of a pulled needle on a beveling wheel at an angle of $45^{\circ}$ for $15-20 \mathrm{~min}$.

NOTE: Exact settings for pulling will vary based on the puller being used.

Thefinal inner diameter of the bevel should be between $20-40 \mu \mathrm{m}$.

b. Coat the inner and external surfaces of the glass capillary with silicone. First dip the 
needles in the siliconizing agent to coat the external surface of the needle andthen backload the siliconizing agent solution into each micropipette to coat the inner surface.

NOTE: Coating of the glass capillaries should be done $24 \mathrm{~h}$ before the implantation experiment. Coating the glass capillaries with silicone provides a chemically inert surface to the glass. If the capillaries are left untreated, the cellsuspension generated in later steps will adhere to the glass and plug the needle.Therefore, coating is necessary and vital to the success of the method.

CAUTION: The siliconizing agent is a ready-made commercially available mixture of heptane and 1,7-dichloro-1,1,3,3,5,5,7,7- octamethyltetrasiloxane (Table 1). It is extremely flammable and acutely toxic. Always handlewith proper PPE inside a fume hood.

i. To backload the needle, load $\sim 5-10 \mu \mathrm{L}$ of the siliconizing agent into a microinjection pipette tip (Table 1). Place the microinjectiontip in the wide end of the pulled glass capillary and position the tip as far down as possible (close to the glass needle tip). Eject the siliconizing agent while slowly removing the loading pipette in order to minimize air bubbles in the needle.

ii. Leave the siliconizing agent in the glass needle for $10 \mathrm{~min}$, remove by aspirating with a new loading pipette and allow needles dry overnight ina fume hood.

c. The morning of the experiment, rinse the glass capillaries with deionized water following the procedure in step $1 \mathrm{a}$ and allow to dry for $3-4 \mathrm{~h}$.

\section{Preparation of solutions}

a. Prepare $5 \mathrm{~mL}$ of trypsin neutralizing solution by supplementing $4.2 \mathrm{~mL}$ of Dulbecco's modified Eagle's medium and Ham's nutrient mixture F12 (DMEM/F12) with $750 \mu \mathrm{L}$ 
of Fetal Bovine Serum (FBS) and $50 \mu \mathrm{L}$ of Penicillin/ Streptomycin. Store at $37^{\circ} \mathrm{C}$ until use

b. Prepare $5 \mu \mathrm{M}$ labeling dye solution by pipetting $5 \mu \mathrm{L}$ of $1 \mathrm{mM}$ stock labeling dye(in dimethyl sulfoxide [DMSO], Table 1) into $995 \mu \mathrm{L}$ of Hank's balanced salt solution (HBSS). Vortex for $1 \mathrm{~min}$ and store at $37^{\circ} \mathrm{C}$ until use.

c. Prepare fresh paraformaldehyde (PFA) by combining $10 \mathrm{~mL}$ of $32 \%$ PFA stock solution with $62 \mathrm{~mL}$ of molecular biology grade water and $8 \mathrm{~mL}$ of 10x Dulbecco's phosphate-buffered saline (DPBS). The final concentration is $4 \%$ PFA in 1x DPBS.

\section{Preparation of host embryos}

a. Incubate fertile chicken eggs in a horizontal orientation in a humidified incubatorat 38 ${ }^{\circ} \mathrm{C}$ until Hamburger and Hamilton (HH) Stage 19 (Hamburger and Hamilton, 1951).

NOTE: The stage chosen for manipulation is flexible and entirely dependentupon the aims of each individual experiment.

b. Score the "flat" end of the egg shell along the egg equator using angled forceps to make a small puncture $>1 \mathrm{~mm}$ in diameter. Insert an $18 \mathrm{G}$ needle with attached $10 \mathrm{~mL}$ syringe through the puncture and remove $\sim 5 \mathrm{~mL}$ of albumin.

NOTE: This anatomical location is external to the "air cell" inside the egg andkeeps albumin from leaking once the puncture is made. This step is recommended as it "drops" the embryo away from the egg shell, preventing potential damage in the subsequent steps.

c. Apply transparent tape to the top of the egg shell. Score with angled forceps andcut a $\sim 2.5 \mathrm{~cm}$ window using curved tenotomy scissors.

d. Inspect and stage the embryo based on criteria established by Hamburger and 
Hamilton ${ }^{10}$ and seal the puncture from step $3 \mathrm{~b}$ with transparent tape.

NOTE: Here, stage HH 19 embryos are used which have 37-40

somites extending into the tail bud. The puncture should not be sealed until after thewindow is opened along the top of the egg (step 3c).

e. Inject $\sim 200 \mu \mathrm{L}$ of India Ink/HBSS mixture (1:5) beneath the embryo using a $32 \mathrm{G}$ needle with attached $1 \mathrm{~mL}$ syringe.

NOTE: India ink provides visual contrast between the embryo and the yolk beneath. Alternative dyes such as neutral red or commercially available cyan fluorescent protein (CFP) or blue fluorescent protein (BFP) fluorescence filtersets can be used to improve contrast.

f. Add $1 \mathrm{~mL}$ of HBSS dropwise onto the embryonic disc and seal windowed shellswith paraffin film. Place eggs back in the humidified incubator until ready for injection.

\section{Isolation of donor tissue}

a. Incubate donor fertile chicken eggs in a humidified incubator at $38{ }^{\circ} \mathrm{C}$ until StageHH 19 (or desired stage).

b. Remove the embryo from the egg and place in a $100 \mathrm{~mm} \times 15 \mathrm{~mm}$ petri dish containing sterile HBSS at room temperature (RT).

c. Surgically microdissect atrial donor tissue from each embryo by first isolating the entire embryonic heart from the embryo and then by isolating the atria from the heart using forceps, tenotomy scissors, and microspatula under a stereo dissecting microscope. Pool in a sterile $1.5 \mathrm{~mL}$ microcentrifuge tube containing $1 \mathrm{~mL}$ of HBSS on ice.

d. Once all donor tissue has been collected, pellet the tissue by centrifugation at 1000 $\times g$ for 5 min at $4{ }^{\circ} \mathrm{C}$ in a fixed-angle microcentrifuge.

\section{Trypsin digestion of donor tissue}


a. Resuspend cell pellets in $1 \mathrm{~mL}$ of prewarmed $0.05 \%$ trypsin-EDTA and incubateat $37{ }^{\circ} \mathrm{C}$ for $15 \mathrm{~min}$ in a shaking heat block at $300 \mathrm{rpm}$. Alternatively, use a waterbath with periodical agitation of the sample.

b. Pipette the digestion solution up and down to break up any remaining tissue, and pellet as in step $4 \mathrm{~d}$.

c. Resuspend the pellet in $1 \mathrm{~mL}$ of the trypsin neutralizing solution and centrifugeas in step 4d.

d. Resuspend the cells in $400 \mu \mathrm{L}$ of red fluorescent labeling dye solution and incubate at 37 ${ }^{\circ} \mathrm{C}$ for $20 \mathrm{~min}$ in a heat block. Alternatively, use a water bath.

e. Once the labeling reaction is finished, pellet the cells as in step $4 \mathrm{~d}$ and washwith $1 \mathrm{~mL}$ of HBSS (number of wash steps can be varied between a and c).

f. Resuspend the labeled, pelleted cells at a concentration of $\sim 50,000$ cells $/ \mu \mathrm{L}$, which generally results in a 5-10 $\mu \mathrm{L}$ working volume depending on the total cellyield.

NOTE: Cell concentrations below $\sim 50,000$ cells $/ \mu \mathrm{L}$ can result in poor injectionefficiency.

\section{In-vivo injection}

a. Backload the cell suspension into a silicone treated glass capillary pipette following the procedures in step $1 \mathrm{~b}$. Mount the pipette into the pressure microinjector apparatus.

b. Remove host embryos from humidified incubator and place in an egg holderunderneath the fluorescent stereo dissecting microscope.

i. Open the vitelline membrane using sterile fine forceps and make a small incision ( $\sim .5-1.0 \mathrm{~mm}$ in length) in the pericardium. Additionalmanipulation/dissection may be needed depending on target region forinjection.

c. Position the microinjector such that the tip of the microinjection needlepenetrates the target tissue. 
i. Pressure inject cells, and use the fluorescent label to determine that implanted cells are present in desired tissue. For typical injections, apply single pulses less than $0.5 \mathrm{~s}$ in duration ranging from $100-400$ hectopascals in pressure.

NOTE: Pulse length and absolute pressure will vary depending on thenumber of cells to be injected and can be modified to suit individual needs.

ii. Retract the microinjector apparatus and remove the egg from the holderafter pressure injecting.

d. Add $1 \mathrm{~mL}$ of warm HBSS dropwise onto the embryo, seal eggs using transparentpacking tape, and incubate in the humidified incubator at $38{ }^{\circ} \mathrm{C}$ for $24 \mathrm{~h}$ post implantation.

\section{Isolation and analysis}

a. Isolate host embryos in RT HBSS using forceps, tenotomy scissors, and microspatula similar to step $4 \mathrm{c}$, and fix in $4 \%$ PFA overnight at $4{ }^{\circ} \mathrm{C}$ with gentlerocking.

b. Wash embryos $3 \times 5 \mathrm{~min}$ in HBSS at RT with gentle rocking, and store in HBSSat $4{ }^{\circ} \mathrm{C}$ for further downstream analysis (microscopy, immunohistochemistry, in-situ hybridization, etc.).

\section{Representative Results}

After $24 \mathrm{~h}$ incubation, the heart and surround tissue of host embryos were isolated, photographed (Figure 23A), and processed for immunofluorescent analysis. In this example,donor atrial myocytes were microinjected into the proepicardium of a similar staged host embryo. The host embryo was then stained with the muscle marker (MF20 green) and 4',6-diamidino-2-phenylindole (DAPI; blue). Injected cells (red) are clearly visible (Figure 23B).Possible adjustments to consider if cells are not visible include: donor tissue was over digested (cells would be unable to attach), labeling dye solution was too dilute, cells were over-washed, or multiple cell divisions resulted in loss of the label.

To confirm that the injected cells in this example were myocardial, we optically sectionedthis embryo using a confocal microscope (Figure 23C-E). The only MF20 positive cells within the 
proepicardium (PE) are the fluorescent red positive cells that were focally implanted.

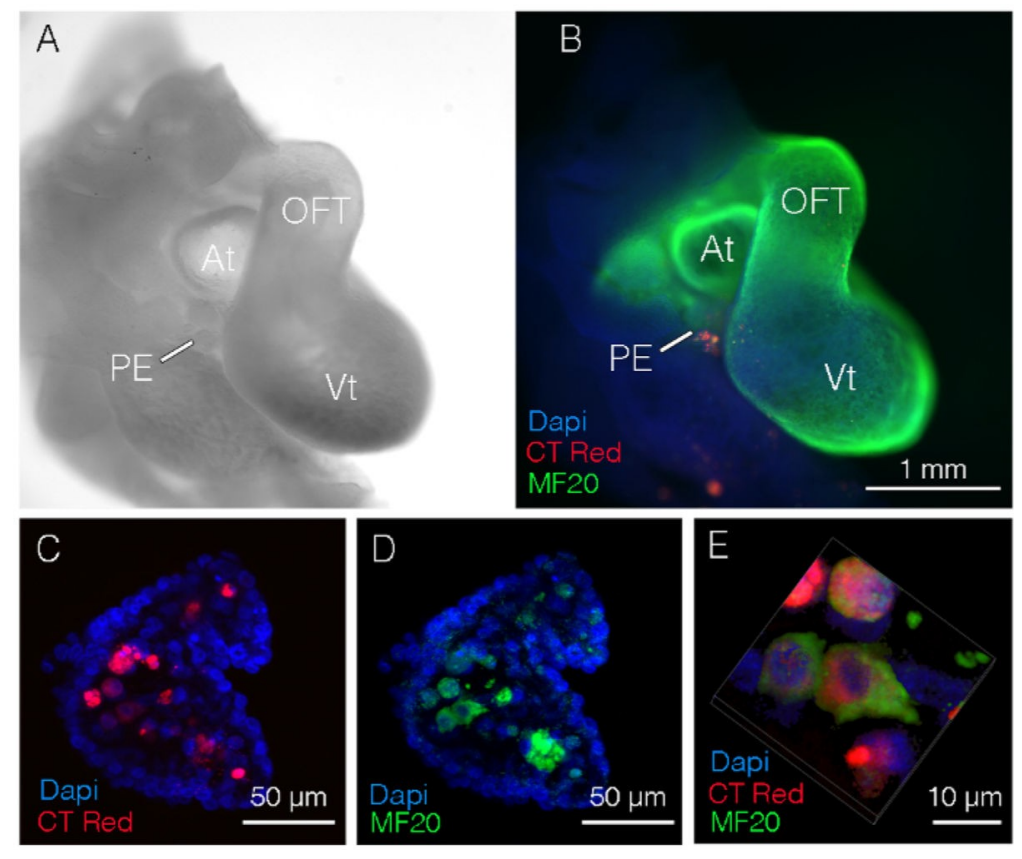

Figure 23: Representative images of embryos isolated 24 hours post injection.

A) Low magnification brightfield image of the trunk region of an E3.5 (HH Stage 19) chickembryo. B) Merged image showing injected cells (red), cardiomyocytes (green), and DAPI.Cells were isolated from the atria and microinjected into the proepicardium. C) High magnification confocal imaging showing labeled cells in the core of the proepicardium. D) High magnification confocal imaging confirming CT Red labeled cells are cardiomyocytes. E) Three-dimensional (3D) reconstruction of injected cells from panels D and E. At, atria;OFT, outflow tract; PE, proepicardium; Vt, ventricle; MF20, Myosin 4.

\section{Discussion}

The ability to define how microenvironmental conditions impact cardiac cell fate specification and lineage stabilization is fundamental to creating a compressive understanding of congenital heart disease as well as to developing efficient protocols forproper maturation of stem cell or somatic cell reprograming-based cardiomyocytes. The protocol outlined above gives investigators the ability to directly assay cardiac cell development under altered in vivo conditions, allowing for cell autonomous maturationprocesses to be separated from paracrine/juxtacrine and/ or hemodynamic cues. When combined with high resolution imaging, genetic analysis, and physiological assays, thistechnique can serve as a powerful complement to existing transgenic models. 
Form a technical stand point, the protocol presented here relies on efficient isolation, labeling, and precise implantation of donor heart cells into host embryonic tissues. The use of a microinjection system greatly aids in the targeting of the donor cells and allows for successful implantation without the need for creating a large engraftment site in the host tissue. Some operational skill is required to perform this technique however, as reduced viability can result if the injection needle is not carefully placed in the target tissue (causingrupture of the heart or local vasculature). Care and thought should also be given to the isolation and labeling steps. Over digestion of the donor tissue can lead to poor implantation efficiency, and transient labeling techniques can limit the time window over which donor cells can be tracked (as cell division can dilute the label).

This technique is highly modifiable and can be adapted for a variety of purposes. For example, donor cells from a large range of tissues and stages can be isolated (though optimization of the enzymatic digestion is required) and can similarly be injected into a variety of host tissues across different stages of development. Similarly, the labeling approach can be modified to track cells across different temporal windows, including the useof fluorescent inorganic semiconductor nanocrystals for longer transient labeling and implantation of quail cells or cells from green fluorescent protein transgenic (GFP+) donor embryos (Bressan et al., 2013) for permeant labeling.

While we currently use this technique for avian implantation studies, we feel that it could beused for a large range of chimeric studies in the future. For example, genetically altered cardiac cells from transgenic organisms could be isolated and microinjected into the avian heart using a very similar protocol. Furthermore, cells differentiated into cardiomyocytes from stems cells or via somatic cell reprograming approaches could be microinjected into the embryonic heart to evaluate their integration into the tissue and/or maturation under in vivo biomechanical conditions. 
Table 2: List of Materials for transfection and in vivo implantation of embryonic cardiomyocytes in the avian embryo protocol

\begin{tabular}{|c|c|c|c|}
\hline Name & Company & $\begin{array}{l}\text { Catalog } \\
\text { Number }\end{array}$ & Comments \\
\hline $1 \mathrm{~mL}$ Insulin Syringe & $\mathrm{BD}$ & 329654 & \\
\hline $1.7 \mathrm{~mL}$ Microtubes, Clear & Genesee Scientific & $24-282$ & \\
\hline $10 \mathrm{ml}$ Syringe & $\mathrm{BD}$ & 305482 & \\
\hline 1000ul Reach Barrier Tip Racked, Sterile & Genesee Scientific & $24-430$ & \\
\hline $15 \mathrm{~mL}$ Centriguge Tubes, Racked & Genesee Scientific & $28-101$ & \\
\hline 1588 Genesis Hova-Bator Incubator & GQF & $\begin{array}{l}81392702122 \\
1\end{array}$ & \\
\hline $18 \mathrm{G} \times 11 / 2$ Needle & $\mathrm{BD}$ & 305196 & \\
\hline $\begin{array}{l}\text { 200ul Barrier Tip Low Binding, Racked, } \\
\text { Sterile }\end{array}$ & Genesee Scientific & $24-412$ & \\
\hline $32 \mathrm{G} \times 1 / 2 "$ Needle & TSK Steriject Air-Tite & TSK3213 & \\
\hline Alchohol Wipes 70\% & $\begin{array}{l}\text { Thermo Fisher } \\
\text { Scientific }\end{array}$ & 19015744 & \\
\hline Angled Forceps & Fine Scientific Tools & $11260-20$ & \\
\hline Backloading Tips & Eppendorf & 930001007 & \\
\hline Black India Ink & KOH-I-NOOR & $3084-\mathrm{F}$ & \\
\hline CellTracker Green CMF & $\begin{array}{l}\text { Thermo Fisher } \\
\text { Scientific }\end{array}$ & C7025 & $\begin{array}{l}1 \mathrm{mM} \text { in } \\
\text { DMSO }\end{array}$ \\
\hline CellTracker Red CMTPX & $\begin{array}{l}\text { Thermo Fisher } \\
\text { Scientific }\end{array}$ & C34552 & $\begin{array}{l}1 \mathrm{mM} \text { in } \\
\mathrm{DMSO}\end{array}$ \\
\hline Centrifuge & Eppendorf & $5424 \mathrm{R}$ & \\
\hline Commercial Grade Packing Tape & Staples & 2619001 & \\
\hline Curved Tenotomy Scissors & Fine Scientific Tools & $14067-11$ & \\
\hline DMEM/F12 & $\begin{array}{l}\text { Thermo Fisher } \\
\text { Scientific }\end{array}$ & $11330-032$ & \\
\hline Name & Company & $\begin{array}{l}\text { Catalog } \\
\text { Number }\end{array}$ & Comments \\
\hline DMSO, anhydrous & $\begin{array}{l}\text { Thermo Fisher } \\
\text { Scientific }\end{array}$ & D12345 & \\
\hline DPBS (10X), no calcium, no magnesium & $\begin{array}{l}\text { Thermo Fisher } \\
\text { Scientific }\end{array}$ & 14025092 & \\
\hline Femtojet $4 \mathrm{i}$ & Eppendorf & 5252000021 & \\
\hline Fetal Bovine Serum & $\begin{array}{l}\text { Thermo Fisher } \\
\text { Scientific }\end{array}$ & $10437-028$ & \\
\hline Hatching Eggs & Pilgrim's Hatchery & -- & \\
\hline $\begin{array}{l}\text { HBSS, calcium, magnesium, no phenol } \\
\text { red }\end{array}$ & $\begin{array}{l}\text { Thermo Fisher } \\
\text { Scientific }\end{array}$ & $14025-092$ & \\
\hline Injectman 4 & Eppendorf & $5192000027 \mathrm{D}$ & \\
\hline
\end{tabular}




\begin{tabular}{|c|c|c|}
\hline Micromanipulator & Leica Microsystems & -- \\
\hline Parafilm & SIGMA & P6543-1EA \\
\hline $\begin{array}{l}\text { Paraformaldehyde } 32 \% \text { in aqueous } \\
\text { solution, EM Grade }\end{array}$ & VWR & $100496-496$ \\
\hline Penicillin/Streptomycin & $\begin{array}{l}\text { Thermo Fisher } \\
\text { Scientific }\end{array}$ & $15140-022$ \\
\hline Petri Dish & Genesee Scientific & $32-107$ \\
\hline Pipette Grinder & Narishige & EG-44 \\
\hline Pipette Puller & HEKA & PIP 6 \\
\hline Scotch Transparent Tape & Staples & 487909 \\
\hline Sigmacote & SIGMA & SL2-25ML \\
\hline Stereo Microscope & Leica & -- \\
\hline ThermoMixer $\mathrm{C}$ & Eppendorf & 5382000023 \\
\hline Thin Wall Glass Capillaries & $\begin{array}{l}\text { World Precision } \\
\text { Instruments }\end{array}$ & TW100F-4 \\
\hline Transfer Pipette & $\begin{array}{l}\text { Thermo Fisher } \\
\text { Scientific }\end{array}$ & 273 \\
\hline Trypsin-EDTA (0.05\%), phenol red & $\begin{array}{l}\text { Thermo Fisher } \\
\text { Scientific }\end{array}$ & $25300-054$ \\
\hline
\end{tabular}




\section{REFERENCES}

Bressan, M., Liu, G., Mikawa, T. (2013). Early mesodermal cues assign avian cardiac pacemaker fate potential in a tertiary heart field. Science. 340 (6133), 744-748.

Bressan, M. et al. (2014). Reciprocal myocardial-endocardial interactions pattern the delay in atrioventricular junction conduction. Development. 141 (21), 4149-4157.

Guo, Y.X. et al. (2017). Analysis of Cardiac Myocyte Maturation Using CASAAV, a Platform for Rapid Dissection of Cardiac Myocyte Gene Function In Vivo. Circulation Research. 120 (12), 18741888.

Guo, Y.X., Pu, W.T. (2018). Genetic Mosaics for Greater Precision in Cardiovascular Research. Circulation Research. 123, 27-29.

Hamburger, V., Hamilton, H.L. (1951). A series of normal stages in the development of the chick embryo. Journal of Morphology. 88 (1), 49-92.

Reinecke, H., Zhang, M., Bartosek, T., Murray, C.E. (1999). Survival, integration, and differentation of cardiomyocyte grafts: a study in normal and injured rat hearts. Circulation. 100, 193-202.

Rojas, S.V. et al. (2017). Transplantation of purified iPSC-derived cardiomyocytes in myocardial infarction. PLOS ONE. 12, e0173222.

Rugh, R. (1962). Experimental embryology; techniques and procedures. 3rd edition, Burgess Pub. Co.

Slack, J.M.W. (2013). Essential developmental biology. 3rd edition, Wiley.

Zhang M et al. (2001). Cardiomyocyte Grafting for Cardiac Repair: Graft Cell Death and Anti-Death Strategies. Journal of Molecular and Cellular Cardiology. 33 (5), 907-921. 